\title{
Deformation problems in Lie groupoids
}

\author{
Cristian Camilo Cárdenas Cárdenas
}

TESE APRESENTADA

$\mathrm{AO}$

Instituto DE MATEMÁticA E EstatístiCA

DA

UNIVERSIDADE DE SÃO PAULO

PARA

OBTENÇÃO DO TÍTULO

$\mathrm{DE}$

DOUTOR EM CIÊNCIAS

Programa: Matemática

Orientador: Prof. Dr. Ivan Struchiner

Durante o desenvolvimento deste trabalho o autor recebeu auxílio financeiro da CAPES/CNPq

São Paulo, março de 2018 
Deformation problems in Lie groupoids

Esta é a versão original da tese elaborada pelo candidato Cristian Camilo Cárdenas Cárdenas, tal como submetida à Comissão Julgadora. 


\section{Deformation problems in Lie groupoids}

Esta versão da tese contém as correções e alterações sugeridas pela Comissão Julgadora durante a defesa da versão original do trabalho, realizada em 20/03/2018. Uma cópia da versão original está disponível no Instituto de Matemática e Estatística da Universidade de São Paulo.

Comissão Julgadora:

- Prof. Dr. Ivan Struchiner (orientador) - IME-USP

- Prof. Dr. Cristián Ortiz - IME-USP

- Prof. Dr. Henrique Bursztyn - IMPA

- Prof. Dr. Rui Loja Fernandes - UI

- Prof. Dr. David Martinez Torres - PUC-Rio 


\section{Acknowledgments}

I sincerely want to thank to my supervisor Ivan Struchiner for introducing me in this motivating field of Mathematics. His constant questions and advises as well as his nice friendship helped me to evolve myself during the years of this work. His patiente and avaliability for talk about Math and non-Math was a stimulating fact for me already since the early years of the doctorate. I am also very grateful to Professor Cristián Ortiz for his valuable advises regarding my mathematical career and for the several discussions about Math. His lectures were always a source of answers and approaches to many questions I often had. I wish to thank to João Mestre for his great help during his several visits to Brazil during these years. My thanks also go to Matias del Hoyo for answering several of my questions during his onemonth visit to IME-USP in the begining of my PhD. I also thank to Rui Loja Fernandes for useful conversations on some key facts of this thesis. I would like to thank the members of the reading committee, professors Henrique Bursztyn, Rui Loja Fernandes, David Martinez Torres, and Cristián Ortiz for taking the time to read this thesis and for their useful comments. I thank the Mathematics Department of University of São Paulo for the stimulating research environment, with several formal and informal seminars I could learn some beautiful Math and give my starting (and more) steps in the process of this research. Also useful were the informal discussions with my colleagues to whom I extend my thanks: Camilo, Fernando E., Fernando S. and Genaro. I wish to thank to the several friends Jeovanny, Laura, Elkin, Wilson, Pablo, Leonardo, Diego, Benigno, Cesar and Thais for the nice moments at USP and at São Paulo we shared, and for the distracting moments of soccer games with some of you. Also my thanks to Marcia, her support was determining in the final stages of this thesis. Una dedicación muy especial va para mi familia, sin ustedes ni su grande apoyo y cariño nada de esto habría sido posible. Last I thank to Capes and CNPq for financial support during these years. 


\section{Resumo}

CÁRDENAS, C. C. C. Problemas de deformação em grupoides de Lie. 2018. 120 f. Tese (Doutorado) - Instituto de Matemática e Estatística, Universidade de São Paulo, São Paulo, 2018.

Nesta tese apresentamos a teoria de deformação de morfismos de grupoides Lie, subgrupoides de Lie e grupoides simpléticos, definimos os correspondentes complexos de deformação que controlam as deformações destas estruturas, e usamos estes complexos para desenvolver o argumento de Moser em cada um destes contextos. Também aplicamos esta teoria ao caso de morfismos de grupos de Lie e subgrupos de Lie obtendo resultados de rigidez de tais estruturas. Ademais, no caso de grupoides simpléticos, definimos uma função entre a cohomologia diferenciável e a cohomologia de deformação do grupoide, que é interpretada como o análogo global da aplicação $i: H_{\pi}^{*}(M) \longrightarrow H_{\text {def }}^{*}\left(\left(T^{*} M\right)_{\pi}\right)$ definida por Crainic and Moerdijk (2004) que relaciona a cohomologia de Poisson da estrutura de Poisson induzida na base $M$ do grupoide com a cohomologia de deformação do algebroide de Lie $\left(T^{*} M\right)_{\pi}$ associado à estrutura de Poisson.

Palavras-chave: Deformações, grupoides de Lie, subgrupoides, grupoides simpléticos. 


\section{Abstract}

CÁRDENAS, C. C. C. Deformation problems in Lie groupoids. 2018. 120 f. Tese (Doutorado) - Instituto de Matemática e Estatística, Universidade de São Paulo, São Paulo, 2018.

In this thesis we present the deformation theory of Lie groupoid morphisms, Lie subgroupoids and symplectic groupoids. The corresponding deformation complexes governing such deformations are defined and used to investigate a Moser argument in each of these contexts. We also apply this theory to the case of Lie group morphisms and Lie subgroups obtaining rigidity results of these structures. Moreover, in the case of symplectic groupoids, we define a map between the differentiable and deformation cohomology of the underlying groupoid, which is regarded as the global counterpart of a map $i: H_{\pi}^{*}(M) \longrightarrow H_{d e f}^{*}\left(\left(T^{*} M\right)_{\pi}\right)$ defined by Crainic and Moerdijk (2004) which relates the (Poisson) cohomology of the Poisson structure on the base $M$ of the groupoid to the deformation cohomology of the Lie algebroid $\left(T^{*} M\right)_{\pi}$ associated to it.

Keywords: Deformations, Lie groupoids, subgroupoids, symplectic groupoids. 


\section{Contents}

1 Introduction 1

2 Deformation of morphisms and subgroupoids $\quad 7$

2.1 Groupoids, algebroids and deformation of Lie groupoids . . . . . . . . . . 7

2.1.1 Deformation theory of Lie groupoids . . . . . . . . . . . . . 8

2.2 Deformations . . . . . . . . . . . . . . . . . . . . . . 12

2.3 Deformation complexes . . . . . . . . . . . . . . . . . . . . . . . 14

2.3.1 Deformation complex of morphisms . . . . . . . . . . . . . . . 14

2.3.2 Deformation complex of Lie subgroupoids . . . . . . . . . . . . . . 15

2.4 Infinitesimal cocycles associated to deformations . . . . . . . . . . . . . 16

2.5 Moser's trick . . . . . . . . . . . . . . . . . . . . . . . . . . . 19

2.6 Simultaneous deformations . . . . . . . . . . . . . . . . . . . 24

2.7 Particular cases and relation between (sub)complexes . . . . . . . . . . 26

$2.7 .1 \mathcal{H}$ and $\mathcal{G}$ fixed . . . . . . . . . . . . . . . . . . . 26

$2.7 .2 \mathcal{G}$ fixed . . . . . . . . . . . . . . . . . . . . 27

2.8 General deformations . . . . . . . . . . . . . . . . . . . . . 28

3 Application: Rigidity of Lie group morphisms and Lie subgroups 31

3.1 Lie group cohomology . . . . . . . . . . . . . . . . . . . 31

3.2 Deformations of morphisms and Lie subgroups . . . . . . . . . . . . . . 32

3.2.1 Relation with deformation complexes and associated cocycles . . . . 35

3.2 .2 Rigidity . . . . . . . . . . . . . . . . . . . 36

4 Deformation of symplectic groupoids $\quad 39$

4.1 VB-groupoids . . . . . . . . . . . . . . . . . . . . . . . . . . . . . . 39

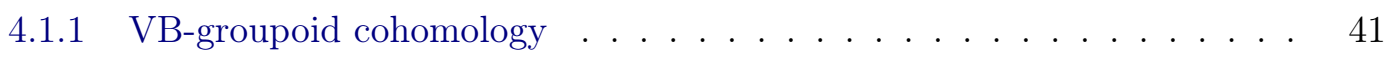

4.2 Deformations . . . . . . . . . . . . . . . . . . . . 42

4.3 Deformation complex of (symplectic) groupoids . . . . . . . . . . . . . 43

4.4 Associated cocycle and Moser's trick . . . . . . . . . . . . . . . . . . 44 
5 Cohomological relation in a symplectic groupoid $\quad \mathbf{5 1}$

5.1 VB-algebroids . . . . . . . . . . . . . . . . . . . . . 51

5.1 .1 Double vector bundles . . . . . . . . . . . . . 52

5.1 .2 Isomorphisms of duals of DVBs . . . . . . . . . . . 55

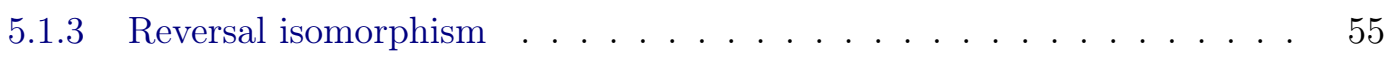

5.1 .4 Linear sections . . . . . . . . . . . . . . . . . 58

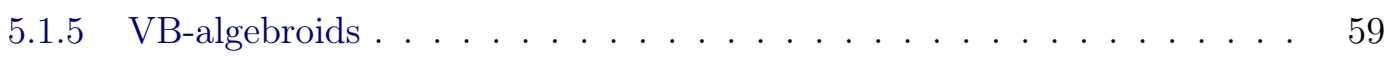

$5.1 .6 \quad$ VB-algebroid complex . . . . . . . . . . . . . . . 61

5.2 Deformation cohomology of Lie algebroids . . . . . . . . . . . . . 61

$5.3 i_{\mathcal{G}}$ and van-Est commutativity $\ldots \ldots \ldots \ldots \ldots \ldots \ldots \ldots$

5.3 .1 Proof of diagram $(I) \ldots \ldots \ldots \ldots 6$

$\begin{array}{ll}\text { Bibliography } & 75\end{array}$ 


\section{Chapter 1}

\section{Introduction}

The aim of this thesis is studying the relation between two areas: Lie groupoids (and related concepts) and deformation theory. More concretely, this work concerns mainly on the deformation theory of several compatible structures associated to Lie groupoids, including symplectic groupoids.

In the last decades, Lie groupoids have obtained a notable relevance in physics and mathematics due to the understanding they brought to many different topics, e.g., Poisson geometry, algebraic geometry, Dirac geometry, non-commutative geometry, among others (Coste et al. (1987), Bursztyn et al. (2004), Bailey and Gualtieri (2016), Weinstein (1996) and references therein). Nowadays, the Lie theory in the Lie groupoids level is a very active research area exploring either its relations with nearby topics or the algebraic/geometrical concepts build on it. Briefly, a Lie groupoid is a (smooth) small category where all its morphisms are invertible. They generalizes the notions of Lie groups (all morphisms with source and target in the same object), Lie group bundles and manifolds (every point (object) of the manifold with a unique morphism starting on it). As with Lie groups, there exist the notions of Lie groupoid morphisms and Lie subgroupoid thought of/defined as (smooth) functors between the underlying categories and (smooth) subcategories of the Lie groupoid (or immersive injective morphisms of Lie groupoids). These notions are, together with symplectic groupoids, the main structures which we will work in this thesis on.

Symplectic groupoids appear notoriously in independent works, motivated by quantization problems, by the late 80's in Karasev (1989); Weinstein (1987); Zakrzewski (1990). They fit as the elements associated to a class of Poisson manifolds, called integrable Poisson structures, and are thought of as the desingularization of Poisson manifolds. A symplectic groupoid consists of a pair $(\mathcal{G}, \omega)$ made of a Lie groupoid and a symplectic form defined on it, which satisfies a certain compatibility condition with respect to the multiplication (composition of the morphisms of the groupoid) of the groupoid.

The deformation problem of these structures offers us another way to understand their properties. In particular, this problem is linked with some cohomology theories defined on 
them which can be interpretated in terms of the infinitesimal properties of the deformations. More generally, in a rough way, the deformation theory is the infinitesimal study of a family of structures in a neighborhood of one of its members. In other words, it is the study of the "derivative" (or first order approximation) of the setting determining the family of structures. It is a general "principle" in deformation theory that such a "derivative", as illustrated since early works on deformations on Lie algebras and complex structures (Kodaira (2006); Nijenhuis and Richardson Jr (1966)) and remarked by many others, is controlled by an appropriate cohomology theory, in the sense that this "derivative" corresponds to a cocycle in that cohomology. With this point of view, such cocycles are thought of as vectors tangent to the families at one of its "points" (members of the family); which leads us heuristically to regard the corresponding cohomology group (of the appropriate degree) as the tangent space to the set of such structures at one fixed structure. Due to this interpretation, the elements of such a cohomology group are also called the infinitesimal deformations of the structure. One well-known application of such infinitesimal deformations is given by the so-called Moser's path method. This technique allows us, based on some regularity conditions on the infinitesimal deformations, and by using the flow of a time-dependent vector field, to say when we have a family (path) of "isomorphic" structures. Pioneers examples of applications of the Moser argument go back to the 60's to the geometry of differential forms; in particular, Moser's method in symplectic geometry is the one most widely known (see e.g McDuff and Salamon (1998)). But more recently, many other interesting applications of this method have appeared, Crainic and Fernandes (2011); Crainic and Struchiner (2013); Marcut (2013), proving some rigidity and linearizability conditions of important structures such as Lie groupoids and Poisson structures; remarking thus the key role of this method in deformation theory.

In this direction, some of the main results presented in this thesis study the infinitesimal behaviour of the deformations of Lie groupoids attached with some additional and compatible data. We show the space of its corresponding infinitesimal deformations and use it to apply a "Moser's trick"-argument in each of these contexts. The results obtained in that way, as stated below, can be (roughly) compared with a basic principle of Calculus: a differentiable function whose derivative vanishes in all points is constant.

Following the setting described above, and under some regularity conditions, the results take the forms below for morphisms and Lie subgroupoids.

Theorem 1 (Morphisms)

Let $\phi:(\mathcal{H}, N) \longrightarrow(\mathcal{G}, M)$ be a morphism between two Lie groupoids with injective imersive base map. Assume that $N$ is compact and $\phi_{\epsilon}$ is a deformation of $\phi$ such that its associated deformation cocycles are exact in a smooth manner. Then $\phi_{\epsilon}$ is a trivial deformation of $\phi$.

Theorem 2 (Subgroupoids)

Let $\mathcal{H} \subset \mathcal{G}$ be a compact Lie subgroupoid of the Lie groupoid $\mathcal{G}$ and $\left\{\mathcal{H}_{\epsilon}\right\}_{\epsilon}$ be a deformation of $\mathcal{H}$ as subgroupoid of $\mathcal{G}$ such that its associated cocycles are exact in a smooth manner. Then 
$\left\{\mathcal{H}_{\epsilon}\right\}_{\epsilon}$ is a trivial deformation of $\mathcal{H}$.

In each of the theorems above, the expression "trivial deformation" has different meanings. It refers to types of deformations which are always canonically defined and which correspond to trivial (constant) curves in the moduli space of the structure being considered. For instance, in the case of morphisms, a trivial deformation of $\phi$ is one of the form $\phi_{\epsilon}=\varphi_{\epsilon} \circ \phi$ where $\varphi_{\epsilon}$ is a smooth one parameter family of inner automorphisms of $\mathcal{G}$. In the case of subgroupoids triviality of a deformation has a similar meaning.

For symplectic groupoids, besides equivalence under automorphisms of the groupoid which are symplectomorphisms, we are led to consider also gauge transformations of the multiplicative symplectic structure induced by pullbacks of closed 2-forms on the base (Chapter 4).

Theorem 3 (Symplectic groupoids I)

Let $(\mathcal{G}, \omega)$ be a compact symplectic groupoid and $\left\{\left(\mathcal{G}_{\epsilon}, \omega_{\epsilon}\right)\right\}_{\epsilon}$ be a deformation of $(\mathcal{G}, \omega)$ such that its associated cocycles are exact. Then $\left\{\left(\mathcal{G}_{\epsilon}, \omega_{\epsilon}\right)\right\}_{\epsilon}$ is determined, up to gauge transformations, by pulling back the structure on $(\mathcal{G}, \omega)$ by a smooth family of diffeomorphisms of $\mathcal{G}$ which comes from the flow of a vector field.

Or slightly modifying the deformation complex involved (and therefore the transgressions of the cocycles) in the case of symplectic groupoids, we describe a groupoid version of the classical Moser theorem:

Theorem 4 (Symplectic groupoids II)

Let $(\mathcal{G}, \omega)$ be a compact symplectic groupoid and $\left\{\left(\mathcal{G}_{\epsilon}, \omega_{\epsilon}\right)\right\}_{\epsilon}$ be a deformation of $(\mathcal{G}, \omega)$ such that its associated cocycles are exact. Then each $\left(\mathcal{G}_{\epsilon}, \omega_{\epsilon}\right)$ is symplectomorphic to $(\mathcal{G}, \omega)$ by a smooth family of isomorphisms of groupoids.

Another important point developed in this thesis, and strongly related to the work described above, is the study of the rigidity properties of the structures being deformed. That is, the resilience of a structure to varying under (small) deformations. Important results in this topic (within the Lie theory context) have been obtained in several works including: Nijenhuis (1968); Nijenhuis and Richardson Jr (1967); Richardson et al. (1967); Richardson Jr et al. (1969); Crainic et al. (2014) for Lie algebras, Lie groups, Lie subalgebras, morphisms of Lie algebras and, more recently, under some compactness conditions, for Lie groupoids Crainic et al. (2015a). As shown in chapter 3 of this thesis, several of the results in chapter 2, when put into the context of Lie groups, give us an alternative way to look at the rigidity question of Lie subgroups and Lie group morphisms (already worked in Coppersmith (1977) and Lee (1974), respectively).

Theorem 5 (Rigidity of morphisms)

Let $\phi: H \longrightarrow G$ be a morphism of Lie groups and assume that $H$ is compact. Then $\phi$ is rigid. 
Theorem 6 (Rigidity of Lie subgroups)

Every compact Lie subgroup is rigid.

The last main result of this thesis treats about symplectic groupoids and integrable Poisson structures. Let us denote $(M, \pi)$ an integrable Poisson manifold. In Crainic and Moerdijk (2004) the authors define a map

$$
i: H_{\pi}(M) \longrightarrow H_{d e f}\left(\left(T^{*} M\right)_{\pi}\right)
$$

which relates the Poisson cohomology and the deformation cohomology of the Lie algebroid $\left(T^{*} M\right)_{\pi}$ associated to that Poisson structure. Note that every deformation of $\pi$ induces a deformation of the cotangent Lie algebroid $\left(T^{*} M\right)_{\pi}$. However, not every deformation of the Lie algebroid $\left(T^{*} M\right)_{\pi}$ is by deformations of the Poisson structure. The deformations of $\left(T^{*} M\right)_{\pi}$ which comes from deformations of $\pi$ give rise to cocycles in the image of $i$. If $\mathcal{G}$ is a symplectic groupoid, we define a map

$$
i_{\mathcal{G}}: H_{\mathrm{diff}}^{*}(\mathcal{G}) \longrightarrow H_{d e f}^{*}(\mathcal{G})
$$

between the differentiable cohomology to the deformation cohomology of the underlying Lie groupoid. This map provides a global interpretation of the map $i$ when $\mathcal{G}$ is a ( $s$-connected) symplectic groupoid integrating $\pi$. The fact that the map $i$ is an infinitesimal manifestation of the map $i_{\mathcal{G}}$ is reflected by the commutativity of the following diagram.

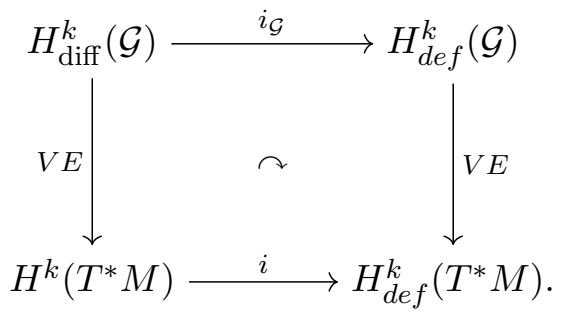

This map $i_{\mathcal{G}}$ also turns out to have a relation with deformation theory of symplectic groupoids: inside of its image are the deformation cocycles of the underlying deformations of Lie groupoids corresponding to deformations by symplectic groupoids.

\section{ORGANIZATION OF THE THESIS}

This thesis is separated into four parts, chapters 2, 3, 4 and 5. In each of the chapters we include a preliminary section which contains the material used in the rest of the chapter and thesis.

Chapter 2 discusses the deformation theory of morphisms of Lie groupoids and Lie subgroupoids. We explain the corresponding deformation cohomologies and the way they encoded the deformations, then we use Moser's argument in these contexts. Also we develop the the- 
ory of simultaneous deformations of a triple consisting of two Lie groupoids and a morphism between them.

In chapter 3, the deformation theory of Lie groupoid morphisms and subgroupoids is used in the context of Lie groups to obtain rigidity results of Lie group morphisms and Lie subgroups.

In chapter 4 we start working with symplectic groupoids. We define deformations of symplectic groupoids and explain how the Bott-Shulman total complex is related to this type of deformations. A groupoid version of the classical Moser's theorem in symplectic geometry is discussed in this chapter.

In chapter 5 , we define a map $i_{\mathcal{G}}: H_{\text {diff }}^{*}(\mathcal{G}) \longrightarrow H_{\text {def }}^{*}(\mathcal{G})$ for a symplectic groupoid $\mathcal{G} \rightrightarrows M$ and we prove that, when $(M, \pi)$ is the (integrable) Poisson structure induced on $M$, this map $i_{\mathcal{G}}$ can be viewed as the global counterpart of the map $i: H_{\pi}^{*}(M) \longrightarrow H_{d e f}^{*}\left(\left(T^{*} M\right)_{\pi}\right)$ defined by Crainic and Moerdijk in (Crainic and Moerdijk (2004)). 


\section{Chapter 2}

\section{Deformation of morphisms and subgroupoids}

\subsection{Groupoids, algebroids and deformation of Lie groupoids}

We start by giving a brief description of the notions involved in this chapter. Let $\mathcal{G} \rightrightarrows M$ be a Lie groupoid, denote by $s, t, m, i$ and $u$ the source, target, multiplication, inversion and unit of $\mathcal{G}$, respectively. We will write $m(g, h)=g h$ e $i(g)=g^{-1}$ when the context is clear, and identify $x \in M$ with the corresponding unity $u(x) \in \mathcal{G}$. For $g \in \mathcal{G}$, write $g: x \rightarrow y$ meaning $s(g)=x$ and $t(g)=y$.

If $g: x \rightarrow y$, there is a right translation

$$
R_{g}: s^{-1}(y) \rightarrow s^{-1}(x), R_{g}(h)=h g
$$

and analogously a left-translation $L_{g}: t^{-1}(x) \rightarrow t^{-1}(y), L_{g}(h)=g h$, between the $t$-fibers. We denote their differentials, respectively, by $r_{g}$ and $l_{g}$. With this, $X \in \mathfrak{X}(\mathcal{G})$ is a rightinvariant vector field if $d s\left(X_{g}\right)=0$ and $X_{h g}=r_{g}\left(X_{h}\right)$ for all $(h, g) \in \mathcal{G}^{(2)}$. Observe that the subset of right-invariant vector fields $\mathfrak{X}^{r}(\mathcal{G}) \subset \mathfrak{X}(\mathcal{G})$ has a $C^{\infty}(M)$-module structure given by $f \cdot X:=\left(t^{*} f\right) X$. Further, $\mathfrak{X}^{r}(\mathcal{G}) \subset \mathfrak{X}(\mathcal{G})$ is a Lie subalgebra.

Just as a Lie group $G$ has an associated Lie algebra $\mathfrak{g}$, Lie groupoids also can be studied infinitesimally giving rise to the notion of Lie algebroid. The Lie algebroid of $\mathcal{G}, A_{\mathcal{G}}$, and is determined (like $\mathfrak{g}$ ) by the Lie algebra of right-invariant vector fields on $\mathcal{G}$. More precisely, $A_{\mathcal{G}}$ is the vector bundle $\left.\left(T^{s} \mathcal{G}\right)\right|_{M}$ over $M$, where $T^{s} \mathcal{G}:=\operatorname{Ker}\left(d s: T \mathcal{G} \longrightarrow s^{*} T M\right)$ and $M \subset \mathcal{G}$ is viewed as the units of $\mathcal{G}$. In this way, there is a map $\Gamma\left(A_{\mathcal{G}}\right) \longrightarrow \mathfrak{X}^{r}(\mathcal{G}), \alpha \longmapsto \vec{\alpha}: g \mapsto r_{g}\left(\alpha_{t(g)}\right)$; which is easily seen to be an isomorphism of $C^{\infty}(M)$-modules inducing then a Lie bracket on $\Gamma\left(A_{\mathcal{G}}\right)$. The vector field $\vec{\alpha}$ is called the right-invariant vector field associated to $\alpha$. The vector bundle $A_{\mathcal{G}}$ is also equipped with a vector bundle map $\rho: A_{\mathcal{G}} \longrightarrow T M$ given by the restriction 
of $d t: T \mathcal{G} \longrightarrow T M$ to $A_{\mathcal{G}} \subset T \mathcal{G}$. The map $\rho$ is called the anchor map of $A_{\mathcal{G}}$.

In other words, the Lie algebroid associated to $\mathcal{G}$ consists of the pair $\left(A_{\mathcal{G}}, \rho\right)$ together with the Lie bracket on sections of $A_{\mathcal{G}}$, induced from that of $\mathfrak{X}^{r}(\mathcal{G})$. With this point of view, we can abstract such a notion of Lie algebroid and to say that a vector bundle $A$ over $M$ is a Lie algebroid if there exist a vector-bundle map $\rho: A \longrightarrow T M$ together with a Lie bracket on the sections of $A$ in such a way that a Leibniz rule is satisfied:

$$
[\alpha, f \beta]_{\Gamma(A)}=f[\alpha, \beta]+L_{\rho(\alpha)}(f) \cdot \beta,
$$

for every $\alpha, \beta \in \Gamma(A)$ and $f \in C^{\infty}(M)$. As the reader may expect, $A_{\mathcal{G}}$ defined as above is an example of a Lie algebroid in this more abstract context. More examples can be found in Moerdijk and Mrcun (2003).

\subsubsection{Deformation theory of Lie groupoids}

The deformation theory of Lie groupoids was explained in Crainic et al. (2015a). In there, the authors developed the main aspects of the theory; among other things, they exhibite the corresponding cohomology attached to deformations of Lie groupoids and use it to give an application to the rigidity problem of Lie groupoids. We recall now some key facts of this construction.

\section{Deformations}

A smooth family of manifolds $\left\{M_{\epsilon} \mid \epsilon \in I\right\}$ is understood as a manifold $\tilde{M}$ together with a submersion $\tilde{\pi}: \tilde{M} \longrightarrow I$, such that $M_{\epsilon}$ is the fiber over $\epsilon$. One also says that the family $\left\{M_{\epsilon} \mid \epsilon \in I\right\}$ is smoothly parametrized by $I$. This notion is the key idea to define deformations of Lie groupoids:

Definition 2.1.1 (Smooth family of Lie groupoids)

A smooth family of Lie groupoids parametrized by a manifold $B$, is given by a Lie groupoid $\tilde{\mathcal{G}} \rightrightarrows \tilde{M}$ and a surjective submersion $\pi$ such that $\pi \circ \tilde{s}=\pi \circ \tilde{t}$

$$
\tilde{\mathcal{G}} \rightrightarrows \tilde{M} \stackrel{\pi}{\rightarrow} B
$$

In this way, $\pi$ determines the family of Lie groupoids $\left\{\mathcal{G}_{b} \mid b \in B\right\}$, where $\mathcal{G}_{b}$ denotes the restricted groupoid over $M_{b}=\pi^{-1}(b)$. One says that the family is proper if $\tilde{\mathcal{G}}$ is proper, i.e., if $\tilde{s} \times \tilde{t}: \tilde{\mathcal{G}} \longrightarrow \tilde{M} \times \tilde{M}$ is a proper map.

Two familes $\tilde{\mathcal{G}} \rightrightarrows \tilde{M} \stackrel{\pi}{\rightarrow} B$ and $\tilde{\mathcal{G}}^{\prime} \rightrightarrows \tilde{M}^{\prime} \stackrel{\pi^{\prime}}{\rightarrow} B$ are isomorphic if there exists an isomorphism of groupoids $(F, f): \tilde{\mathcal{G}} \rightarrow \tilde{\mathcal{G}}^{\prime}$ compatible with the submersions $\pi$ and $\pi^{\prime}$ in the sense that 
$\pi^{\prime} \circ f=\pi$. This isomorphism $F$ can be thought of as a family of isomorphisms $F_{b}: \mathcal{G}_{b} \longrightarrow \mathcal{G}_{b}^{\prime}$ smoothly parametrized by $B$.

Definition 2.1.2 (Deformation of Lie groupoids)

Let $\mathcal{G} \rightrightarrows M$ be a Lie groupoid with structural maps $s, t, m, i, u$. A deformation of $\mathcal{G}$ is a smooth family of Lie groupoids $\tilde{\mathcal{G}}$ parametrized by an open interval I containing zero,

$$
\tilde{\mathcal{G}}=\left\{\mathcal{G}_{\epsilon} \rightrightarrows M_{\epsilon}: \epsilon \in I\right\}
$$

such that $\mathcal{G}_{0}=\mathcal{G}$. We denote the structural maps of $\mathcal{G}_{\epsilon}$ by $s_{\epsilon}, t_{\epsilon}, m_{\epsilon}, i_{\epsilon}, u_{\epsilon}$.

The deformation $\tilde{\mathcal{G}}$ of $\mathcal{G}$ is called strict if $\left(\mathcal{G}_{\epsilon}, M_{\epsilon}\right)=(\mathcal{G}, M)$ as manifolds, that is, we only deform the structural maps of $\mathcal{G}$. In other words, $(\tilde{\mathcal{G}} \rightrightarrows \tilde{M}, \tilde{s}, \tilde{t}) \cong\left(\mathcal{G} \times I \rightrightarrows M \times I, s_{\epsilon} \times \epsilon, t_{\epsilon} \times \epsilon\right)$. In such a case, we can assume $\tilde{\mathcal{G}}=\mathcal{G} \times I$ and it is said to be s-constant if $s_{\epsilon}$ does not depend on $\epsilon$. The deformation such that $\mathcal{G}_{\epsilon}=\mathcal{G}$ as groupoids is called the constant deformation of $\mathcal{G}$. Two deformations $\tilde{\mathcal{G}}=\left\{\mathcal{G}_{\epsilon} \rightrightarrows M_{\epsilon}: \epsilon \in I\right\}$ and $\tilde{\mathcal{G}}^{\prime}=\left\{\mathcal{G}_{\epsilon}^{\prime} \rightrightarrows M_{\epsilon}^{\prime}: \epsilon \in I^{\prime}\right\}$ are locally equivalent if there exist a family of isomorphisms of groupoids $F_{\epsilon}: \mathcal{G}_{\epsilon} \longrightarrow \mathcal{G}_{\epsilon}^{\prime}$, smoothly parametrized by $\epsilon$ in a open interval containing zero (contained in $I \cap I^{\prime}$ ), such that $F_{0}=I d_{\mathcal{G}}$.

Remark 2.1.3 Consider two locally equivalent deformations $\tilde{\mathcal{G}}$ and $\tilde{\mathcal{G}}^{\prime}$. For simplicity and because around $\mathcal{G}_{0}$ the families $\tilde{\mathcal{G}}$ and $\tilde{\mathcal{G}}^{\prime}$ are isomorphic, we will just say that $\tilde{\mathcal{G}}$ and $\tilde{\mathcal{G}}^{\prime}$ are equivalent deformations of $\mathcal{G}_{0}$ (even if $I \neq I^{\prime}$ ).

With the convention of the last remark, the deformation $\tilde{\mathcal{G}}$ is called trivial if it is equivalent to the constant deformation.

\section{Remark 2.1.4 (Fibrations)}

As pointed out in del Hoyo and Fernandes (2016), a deformation of $\mathcal{G}$ also can be regarded in terms of fibrations of Lie groupoids. The data $\tilde{\mathcal{G}} \rightrightarrows \tilde{M} \stackrel{\pi}{\rightarrow} B$ involved in the definition of a deformation of $\mathcal{G}$ takes the form

$$
\phi:(\tilde{\mathcal{G}} \rightrightarrows \tilde{M}) \longrightarrow(I \rightrightarrows I)
$$

where $\phi$ is a fibration of Lie groupoids. In this sense, a strict deformation can be though of as a fibration $\phi$ where the maps between the arrows and the objects are locally trivial. In fact, two trivializations $F_{1}: \tilde{\mathcal{G}} \longrightarrow \mathcal{G} \times I$ and $F_{0}: \tilde{M} \longrightarrow M \times I$ induce a family of Lie groupoid structures $\left\{\mathcal{G}_{\epsilon}\right\}_{\epsilon \in I}$ on the manifold $\mathcal{G}=\tilde{\mathcal{G}}_{0}$. For instance, the deformation of the source map is determined by $\left(s_{\epsilon}(g), \epsilon\right)=F_{0} \circ \tilde{s} \circ F_{1}^{-1}(g, \epsilon)$.

Interesting examples of deformations of Lie groupoids are considered in (del Hoyo and Fernandes (2016), p. 16). As a manner of illustration we sketch here some of them. 


\section{Examples 2.1.5}

1. Let $G=\mathbb{R}^{2}$. Consider the family of Lie groups $G \times \mathbb{R} \longrightarrow \mathbb{R},(g, \epsilon) \mapsto \epsilon$ given by

$$
\left(x_{1}, y_{1}\right) \cdot{ }_{\epsilon}\left(x_{2}, y_{2}\right):=\left(x_{1}+x_{2}, y_{1}+e^{x_{1} \epsilon} y_{2}\right) .
$$

Due to the fact that for $\epsilon \neq 0$ the multiplication $\cdot{ }_{\epsilon}$ is non-abelian, this is a non-trivial deformation of $G$.

2. Consider the family of Lie group actions of $\mathbb{R}$ on $\mathbf{T}^{2}:=\mathbb{R}^{2} / \mathbb{Z}^{2}$, given by:

$$
r \cdot \epsilon\left(x_{1}, x_{2}\right):=\left(x_{1}+r, x_{2}+\epsilon r\right) .
$$

Then, if $G:=\mathbb{R} \times \mathbf{T}^{2}$ such a family of actions can be seen as a family of action groupoids $G \times \mathbb{R} \longrightarrow \mathbb{R}$. This is of course a non-trivial deformation of $G$ since the topology of the orbits varies with $\epsilon$.

\section{Cohomology}

The fundamental fact of the deformation complex of a Lie groupoid $\mathcal{G},\left(C_{\text {def }}^{*}(\mathcal{G}), \delta_{\mathcal{G}}\right)$, is that it governs deformations of $\mathcal{G}$. More precisely, to every deformation one associates a cohomology class in $H_{d e f}^{2}(\mathcal{G})$, and this correspondence has a natural relation with equivalent deformations of $\mathcal{G}$. The deformation complex is defined as follows.

For any $k \in \mathbb{N}$, consider $\mathcal{G}^{(k)}=\left\{\left(g_{1}, \ldots, g_{k}\right): s\left(g_{i}\right)=t\left(g_{i+1}\right)\right\}$ the manifold of $k$-strings of composable arrows, and assume $\mathcal{G}^{(0)}=M$. The space of $k$-cochains $C_{d e f}^{k}(\mathcal{G})$ is given by

$$
C_{d e f}^{k}(\mathcal{G})=\left\{c: \mathcal{G}^{(k)} \rightarrow T \mathcal{G} \mid c\left(g_{1}, \ldots, g_{k}\right) \in T_{g_{1}} \mathcal{G} \text { and } c \text { is s-projectable }\right\}
$$

where $s$-projectable means that $d s \circ c\left(g_{1}, \ldots, g_{k}\right)=: s_{c}\left(g_{2}, \ldots, g_{k}\right)$ does not depend on $g_{1}$. The differential of $c$ is defined by

$$
\begin{aligned}
(\delta c)\left(g_{1}, \ldots, g_{k+1}\right): & =-d \bar{m}\left(c\left(g_{1} g_{2}, g_{3}, \ldots, g_{k+1}\right), c\left(g_{2}, \ldots, g_{k+1}\right)\right)+ \\
& +\sum_{i=2}^{k}(-1)^{k} c\left(g_{1}, \ldots g_{i} g_{i+1}, \ldots, g_{k+1}\right)+(-1)^{k+1} c\left(g_{1}, \ldots, g_{k}\right),
\end{aligned}
$$

where $\bar{m}: \mathcal{G}_{s} \times{ }_{s} \mathcal{G} \longrightarrow \mathcal{G}, \bar{m}(g, h)=g h^{-1}$ is the division map of $\mathcal{G}$.

For $k=0, C_{d e f}^{0}(\mathcal{G}):=\Gamma(A)$ with differential defined by

$$
\delta \alpha=\vec{\alpha}+\overleftarrow{\alpha} \in C_{d e f}^{1}(\mathcal{G})
$$

where $\overleftarrow{\alpha}$ is the left-invariant vector field on $\mathcal{G}$ associated to $\alpha$ defined by $\overleftarrow{\alpha}(g):=l_{g}\left(\operatorname{di}\left(\alpha_{s(g)}\right)\right)$ Note that a section of $A$ can be viewed as a map $c: \mathcal{G}^{(0)} \longrightarrow T \mathcal{G}$, with $c\left(1_{x}\right) \in T_{1_{x}} \mathcal{G}$ such 
that $d s \circ c=0$.

This data in fact defines a cohomology $\left(\delta^{2}=0\right)$ and $H_{\text {def }}^{*}(\mathcal{G})$ denotes the deformation cohomology of $\mathcal{G}$.

In this way, for instance, one can describe explicitly the cohomology class $\left[\xi_{0}\right] \in H_{d e f}^{2}(\mathcal{G})$ associated to an $s$-constant deformation of $\mathcal{G}$ by

$$
\xi_{0}(g, h):=\left.\frac{d}{d \epsilon}\right|_{\epsilon=0} \bar{m}_{\epsilon}\left(m_{0}(g, h), h\right), \quad \xi_{0} \in C_{d e f}^{2}(\mathcal{G})_{c l}
$$

where $m_{0}$ denotes the multiplication of $\mathcal{G}=\mathcal{G}_{0}$. The fact that $\xi_{0}$ is a cocycle is implied

from applying $\left.\frac{d}{d \epsilon}\right|_{\epsilon=0}$ to the associativity property of every $\bar{m}_{\epsilon}$. The element $\xi_{0}$ is called the deformation cocycle of the deformation of $\mathcal{G}$. For deformations which are not necessarily $s$ constant, a slightly different approach is used, yielding however a non canonical 2-cocycle, instead of that one gets (canonically) a 2-cohomology class, which does not depend on the equivalence class of the deformation (see Section 5.4 in Crainic et al. (2015a)).

Of remarkable importance is the transgression of the 2-cocycle $\xi_{0}$; when it exists, it plays a determining role in the rigidity problem of Lie groupoids, as we explained below.

\section{Moser's trick (towards rigidity):}

With the help of the deformation complex one can satisfactorily solve the rigidity question for Lie groupoids. One key step in this direction is given by the following proposition, which has the same essence as that of the classical Moser's theorem of symplectic geometry (see e.g. McDuff and Salamon (1998) p. 93).

Proposition 2.1.6 Crainic et al. (2015a) Let $\tilde{\mathcal{G}}=\left\{\mathcal{G}_{\epsilon}: \epsilon \in I\right\}$ be an s-constant deformation of $\mathcal{G}$. Consider the induced cocycles at each time $\epsilon, \xi_{\epsilon} \in C_{\text {def }}^{2}\left(\mathcal{G}_{\epsilon}\right)$, defined in analogous way to $\xi_{0}$ above. Assume that for every $\epsilon$ small enough, there exists $X^{\epsilon} \in C_{d e f}^{1}\left(\mathcal{G}_{\epsilon}\right)$ such that

$$
\delta_{\epsilon}\left(X^{\epsilon}\right)=\xi_{\epsilon}
$$

and that the resulting time-dependent vector field on $\mathcal{G}, X:=\left\{X^{\epsilon}\right\}_{\epsilon}$, is smooth. Then, for $\epsilon_{1}$ and $\epsilon_{2}$ close to 0 , the time-dependent flow $\psi_{X}^{\epsilon_{2}, \epsilon_{1}}$ is a locally defined morphism from $\mathcal{G}_{\epsilon_{1}}$ to $\mathcal{G}_{\epsilon_{2}}$ covering the flow of $\left\{V^{\epsilon}:=d s\left(X^{\epsilon}\right)\right\}_{\epsilon}$.

Additionally, if $\mathcal{G}$ is proper, $\psi_{X}^{\epsilon_{2}, \epsilon_{1}}(g)$ is defined if and only if $\psi_{V}^{t, s}(s(g))$ and $\psi_{V}^{t, s}(t(g))$ are defined.

This proposition tells us the conditions under which one finds a flow compatible with the variations of the structural maps of $\mathcal{G}$. However, by considering the structural maps of the total groupoid $\tilde{\mathcal{G}}$, it is obtained the following equivalent version of the proposition. 
Proposition 2.1.7 Crainic et al. (2015a) Consider an s-constant deformation as above. A one-parameter family $X^{\epsilon}$ of vector fields on $\mathcal{G}$ satisfies the cocycle equations (2.1) if and only if the induced vector field on $\tilde{\mathcal{G}}$,

$$
\tilde{X}(g, \epsilon)=\left(X^{\epsilon}(g), 0\right)+\frac{\partial}{\partial \epsilon} \in \mathfrak{X}(\mathcal{G} \times I),
$$

is multiplicative.

In this way, one knows that the flow of $\tilde{X}$ (when uniformly defined) is given by automorphisms of $\tilde{\mathcal{G}}$ (Mackenzie and Xu (1997), Prop. 3.5); and the rigidity question of Lie groupoids is essentially solved by finding a complete vector field like $X$ (or $\tilde{X}$ ) above (Crainic et al. (2015a) Thm. 7.1).

Our goal now is to develop the analogous (three) steps above (deformations, cohomology and Moser's trick) when working with the deformation theory of Lie groupoid morphisms and Lie subgroupoids.

\subsection{Deformations}

When talking about deformations of a structure it is usual to think of a smoothly parametrized family of such structures. For Lie groupoids, as seen in the previous section, one way to formalize the smoothness of such a family is to look at it as the fibers of a submersion. For morphisms, however, because Lie groupoid morphisms can be regarded simply as functions, this notion has a simpler description. Given $\mathcal{H}$ and $\mathcal{G}$ two Lie groupoids and $\phi: \mathcal{H} \rightarrow \mathcal{G}$ a Lie groupoid morphism, we call the family $\left\{\phi_{\epsilon}\right\}_{\epsilon \in I}$ a deformation of $\phi$ ( $I$ an interval containing the origin) if this is a smooth family of morphisms $\phi_{\epsilon}: \mathcal{H} \rightarrow \mathcal{G}$ with $\phi_{0}=\phi$. If $\left\{\epsilon \mapsto \sigma_{\epsilon}\right\}$ is a smooth family of bisections of $\mathcal{G}$ (i.e. smooth maps $\sigma: M \longrightarrow \mathcal{G}$ such that $s \circ \sigma=i d_{M}$ and $t \circ \sigma$ is a diffeomorphism of $M$ ) with $\sigma_{0}=1_{\mathcal{G}}$, conjugating $\phi$ with each $\sigma_{\epsilon}$ yields a deformation of $\phi, \phi_{\epsilon}:=I_{\sigma_{\epsilon}} \circ \phi$ (i.e., $\left.\phi_{\epsilon}(h)=\sigma_{\epsilon}(t(\phi(h))) \phi(h) \sigma_{\epsilon}(s(\phi(h)))^{-1}\right)$. Such a deformation is called a trivial deformation of $\phi$. Also, we say that two deformations of $\phi,\left\{\phi_{\epsilon}\right\}_{\epsilon}$ and $\left\{\psi_{\epsilon}\right\}_{\epsilon}$, are equivalent if there exist a smooth family of bisections $\left\{\epsilon \mapsto \sigma_{\epsilon}\right\}_{\epsilon}$ of $\mathcal{G}$, with $\sigma_{0}=1_{\mathcal{G}}$, such that $\psi_{\epsilon}=I_{\sigma_{\epsilon}} \circ \phi_{\epsilon}$, for all $\epsilon$.

Example 2.2.1 Let $\phi_{\epsilon}: \mathbb{R} \longrightarrow S^{1} \times S^{1} \subset \mathbb{C}^{2}$ be the family of morphisms given by

$$
\phi_{\epsilon}(r):=\left(e^{i r}, e^{i r(1+\epsilon \pi)}\right)
$$

This family can be seen as a non-trivial deformation of the non-injective morphism $\phi_{0}$ since for any $\epsilon \in \mathbb{Q} \backslash\{0\}$ the morphism $\phi_{\epsilon}$ is injective.

\section{Remark 2.2.2 (Fibrations)}

A deformation of $\phi_{0}: \mathcal{H} \longrightarrow \mathcal{G}$ can be equivalently described by a morphism $\Phi$ between the 
trivial fibrations $(\mathcal{H} \times I \rightrightarrows N \times I \longrightarrow I \rightrightarrows I)$ and $\mathcal{G} \times I \rightrightarrows M \times I \longrightarrow I \rightrightarrows I)$ covering the identity such that restricted to the fiber over 0 is $\phi_{0}$. We denote by $\bar{\Phi}$ the induced base-map between the groupoids $\mathcal{H} \times I$ and $\mathcal{G} \times I$.

We introduce now the notion of deformation of Lie subgroupoids. In order to talk about the smoothness of a family $\left\{\mathcal{H}_{\epsilon} \subset \mathcal{G}\right\}_{\epsilon}$ of Lie subgroupoids, we think of each $\mathcal{H}_{\epsilon}$ coming from the fiber of a submersion, as in the case of Lie groupoids. In this way, a deformation of $\mathcal{H}$ as subgroupoid will be such smooth family with $\mathcal{H} \subset \mathcal{G}$ being the Lie subgroupoid "over" zero. More concretely, we have

Definition 2.2.3 (Deformations of Lie subgroupoids)

A strict deformation of the Lie subgroupoid $\mathcal{H} \subset \mathcal{G}$ consists of a strict deformation of $\mathcal{H}$ as Lie groupoid, $\tilde{\mathcal{H}}=\mathcal{H} \times I$ and a morphism, $\phi: \tilde{\mathcal{H}} \longrightarrow \mathcal{G}$, between the Lie groupoids $\tilde{\mathcal{H}}$ and $\mathcal{G}$, which is an injective immersion when restricted to the fibers $\tilde{\mathcal{H}}_{\epsilon}$ with $\left.\phi\right|_{\tilde{\mathcal{H}}_{0}}=\iota: \mathcal{H} \hookrightarrow \mathcal{G}$. Analogously we define the $s$-constant deformations of $\mathcal{H} \subset \mathcal{G}$ as subgroupoid. The deformation such that $\tilde{\mathcal{H}}=\mathcal{H} \times I$ is the constant deformation as Lie groupoid and $\left.\phi\right|_{\tilde{\mathcal{H}}_{\epsilon}}=\iota$, for all $\epsilon$, is called the constant deformation of $\mathcal{H}$ as Lie subgroupoid of $\mathcal{G}$.

Remark 2.2.4 (Fibrations)

Note that, equivalently, a deformation of $\mathcal{H} \subset \mathcal{G}$ as Lie subgroupoid is an injective immersive morphism $\tilde{\phi}: \tilde{\mathcal{H}} \longrightarrow \mathcal{G} \times I$ between the fibrations over $I \rightrightarrows I$, such that when restricted to the fiber over $0 \in I$ it is the inclusion map, i.e. $\left.\tilde{\phi}\right|_{\tilde{\mathcal{H}}_{0}}=\iota \times\{0\}: \mathcal{H} \hookrightarrow \mathcal{G} \times\{0\}$.

Remark 2.2.5 Similarly we can define general deformations of Lie subgroupoids (that is, non necessarily strict deformations), it requires to take general deformations of $\mathcal{H}$ as Lie groupoid (Definition 2.1.2 above).

By abuse of notation, we will denote by $\mathcal{H}_{\epsilon}:=\phi(H \times\{\epsilon\}) \subset \mathcal{G}$ the image of each fiber $\tilde{\mathcal{H}}_{\epsilon}$ inside $\mathcal{G}$ and we will often write $\left\{\mathcal{H}_{\epsilon}\right\}$ or $\left\{\mathcal{H}_{\epsilon}\right\}_{\phi}$ to denote a deformation of $\mathcal{H}$ as Lie subgroupoid of $\mathcal{G}$. With this, the constant deformation of $\mathcal{H}$ as a subgroupoid satisfies $\mathcal{H}_{\epsilon}=\mathcal{H} \subset \mathcal{G}$ for all $\epsilon$.

Convention: Observe now that given a Lie subgroupoid $\mathcal{H} \subset \mathcal{G}$, one can deform it as a Lie groupoid and as a Lie subgroupoid; in this context $\left\{\tilde{H}_{\epsilon}\right\}_{\epsilon}$ will denote the deformation as a Lie groupoid.

Example 2.2.6 There are some simple ways to produce examples of deformations; as with deformations of morphisms, we can take a smooth family of bisections of $\mathcal{G}$ and to obtain a deformation of a subgroupoid $\mathcal{H}$ looking at the images $I_{\sigma_{\epsilon}}(\mathcal{H})$ for every $\epsilon$. Or more generally, one can take a smooth family of automorphisms $\left\{F_{\epsilon}\right\}_{\epsilon}$ of $\mathcal{G}$, with $F_{0}=I d_{\mathcal{G}}$, and look at the images $F_{\epsilon}(\mathcal{H}) \subset \mathcal{G}$, for every $\epsilon$.

In a certain sense, which will be clear with Theorems 2.5.6 and 2.5.8, these two types of deformations are the simplest ones. 
Strictly speaking, a Lie subgroupoid is an equivalence class of injective immersive morphisms (represented by a subset of the ambient groupoid). In the context of deformations of Lie subgroupoids this implies the situation of two deformations $\{(\tilde{\mathcal{H}}, \phi)\}$ and $\left\{\left(\tilde{\mathcal{H}}^{\prime}, \psi\right)\right\}$ to have the same images inside $\mathcal{G}\left(\left(\phi_{\epsilon}\left(\tilde{\mathcal{H}}_{\epsilon}\right)=\psi_{\epsilon}\left(\tilde{\mathcal{H}}_{\epsilon}^{\prime}\right)\right.\right.$ for all $\left.\epsilon\right)$. Geometrically, we expect these two deformations to be "the same". More generally, we have the following notion of equivalence of deformations.

Definition 2.2.7 (Equivalence of deformations)

We say that two deformations $\left\{\mathcal{H}_{\epsilon}\right\}$ and $\left\{\mathcal{H}_{\epsilon}^{\prime}\right\}$ of $\mathcal{H} \subset \mathcal{G}$ are equivalent if there exists a smooth family of bisections $\left\{\sigma_{\epsilon}\right\}$ of $\mathcal{G}$ with $\sigma_{0}=1_{\mathcal{G}}$, such that $\mathcal{H}_{\epsilon}^{\prime}=I_{\sigma_{\epsilon}}\left(\mathcal{H}_{\epsilon}\right)$ for every $\epsilon$ small enough. A deformation is said to be trivial if it is equivalent to the constant deformation. That is, a deformation is trivial when it is of the form $\mathcal{H}_{\epsilon}=I_{\sigma_{\epsilon}}(\mathcal{H})$, for some family of bisections with $\epsilon$ small enough.

Remark 2.2.8 Note that, due to the injectivity of $\phi_{\epsilon}:=\left.\phi\right|_{\tilde{H}_{\epsilon}}$ in definition 2.2.3, two deformations $\left\{\mathcal{H}_{\epsilon}\right\}_{\phi}$ and $\left\{\mathcal{H}_{\epsilon}^{\prime}\right\}_{\psi}$ are equivalent if, and only if, there exists a unique family of isomorphisms $\left\{F_{\epsilon}: \mathcal{H}_{\epsilon} \rightarrow \mathcal{H}_{\epsilon}^{\prime}\right\}$ such that

$$
\psi_{\epsilon} \circ F_{\epsilon}=I_{\sigma(\epsilon)} \circ \phi_{\epsilon}, \text { for } \epsilon \text { small enough }
$$

Since every $\psi_{\epsilon}$ is an immersion, such a family is smooth.

\subsection{Deformation complexes}

In this section we define the cohomology theories corresponding to each type of the deformations introduced above.

\subsubsection{Deformation complex of morphisms}

The deformation complex of a morphism was briefly discussed in Crainic et al. (2015a). We recall now its definition. Let $\left(\mathcal{H} \rightrightarrows N, s^{\prime}, t^{\prime}\right)$ and $(\mathcal{G} \rightrightarrows M, s, t)$ be two Lie groupoids and $(\phi, \varphi): \mathcal{H} \longrightarrow \mathcal{G}$ a morphism between them.

For any $k \in \mathbb{N}$, consider $\mathcal{H}^{(k)}=\left\{\left(h_{1}, \ldots, h_{k}\right): s^{\prime}\left(h_{i}\right)=t^{\prime}\left(h_{i+1}\right)\right\}$ the manifold of $k$-strings of composable arrows of $\mathcal{H}$ and $\mathcal{H}^{(0)}=N$. The space of $k$-cochains $C_{d e f}^{k}(\phi)$ is given by

$$
C_{\text {def }}^{k}(\phi)=\left\{c: \mathcal{H}^{(k)} \rightarrow T \mathcal{G} \mid c\left(h_{1}, \ldots, h_{k}\right) \in T_{\phi\left(h_{1}\right)} \mathcal{G} \text { and } c \text { is } s \text {-projectable }\right\}
$$

where $s$-projectable means that the $s$-projection of $c, d s \circ c\left(h_{1}, \ldots, h_{k}\right)=: s_{c}\left(h_{2}, \ldots, h_{k}\right) \in$ 
$T_{t\left(\phi\left(h_{2}\right)\right)} M$, does not depend on $h_{1}$. The differential of $c$ is defined by

$$
\begin{aligned}
\left(\delta_{\phi} c\right)\left(h_{1}, \ldots, h_{k+1}\right): & =-d \bar{m}_{\mathcal{G}}\left(c\left(h_{1} h_{2}, h_{3}, \ldots, h_{k+1}\right), c\left(h_{2}, \ldots, h_{k+1}\right)\right)+ \\
& +\sum_{i=2}^{k}(-1)^{k} c\left(h_{1}, \ldots h_{i} h_{i+1}, \ldots h_{k+1}\right)+(-1)^{k+1} c\left(h_{1}, \ldots, h_{k}\right),
\end{aligned}
$$

where $\bar{m}_{\mathcal{G}}: \mathcal{G}_{s} \times{ }_{s} \mathcal{G} \longrightarrow \mathcal{G}, \bar{m}_{\mathcal{G}}(g, h)=g h^{-1}$ is the division map of $\mathcal{G}$.

For $k=0, C_{d e f}^{0}(\phi):=\Gamma\left(\varphi^{*} A_{\mathcal{G}}\right)$ and the differential is

$$
\delta_{\phi} \alpha=\vec{\alpha}+\overleftarrow{\alpha} \in C_{d e f}^{1}(\phi)
$$

where $\vec{\alpha}(h)=r_{\phi(h)}\left(\alpha_{t(h)}\right)$ and $\overleftarrow{\alpha}(h)=l_{\phi(h)}\left(\operatorname{di}\left(\alpha_{s(h)}\right)\right)$

The fact that $\delta_{\phi}^{2}=0$ can be shown in a similar way to the proof that $\delta=0$ for the deformation complex of Lie groupoids; so $\delta_{\phi}$ in fact defines a cohomology and $H_{d e f}^{*}(\phi)$ denotes the deformation cohomology of $\phi$. Observe that $H_{d e f}^{*}\left(I d_{\mathcal{G}}\right)=H_{d e f}^{*}(\mathcal{G})$.

Remark 2.3.1 Note that there are natural cochain maps between the deformation complexes (of Lie groupoids and morphisms):

$$
C_{\text {def }}^{k}(\mathcal{H}) \stackrel{\phi_{*}}{\rightarrow} C_{d e f}^{k}(\phi) \stackrel{\phi^{*}}{\leftarrow} C_{d e f}^{k}(\mathcal{G})
$$

defined by

$$
\begin{gathered}
\left(\phi_{*} c\right)\left(h_{1}, \ldots, h_{k}\right):=(d \phi)_{h_{1}}\left(c\left(h_{1}, \ldots h_{k}\right)\right), \quad c \in C_{d e f}^{k}(\mathcal{H}) ; \text { and } \\
\left(\phi^{*} \alpha\right)\left(h_{1}, \ldots, h_{k}\right):=\alpha\left(\phi\left(h_{1}\right), \ldots, \phi\left(h_{k}\right)\right), \quad \alpha \in C_{d e f}^{k}(\mathcal{G}) .
\end{gathered}
$$

In the case $k=0$ the cochain-map $\phi^{*}$ is denoted by $\varphi^{*}$ (recall that $\varphi$ is the induced map on the units), to make sense with the definition of $C_{\text {def }}^{0}(\phi)=\Gamma\left(\varphi^{*} A_{\mathcal{G}}\right)$. Similarly, if $\mathcal{K} \stackrel{\psi}{\rightarrow} \mathcal{H}$ is another Lie groupoid morphism, one can define a cochain-map $\psi^{*}: C_{d e f}^{*}(\phi) \longrightarrow C_{d e f}^{*}(\phi \circ \psi)$.

Remark 2.3.2 As a special case, if $\phi$ above is bijective, there is an inverse $\phi_{\#}$ of $\phi^{*}$,

$$
\phi_{\#}: C_{d e f}^{*}(\phi) \longrightarrow C_{d e f}^{*}(\mathcal{G}), \phi_{\#}(\hat{T})_{\left(g_{1}, \ldots, g_{k}\right)}:=\hat{T}_{\left(\phi^{-1}\left(g_{1}\right), \ldots, \phi^{-1}\left(g_{k}\right)\right)} .
$$

Similarly, if $\phi^{*}$ is the map between deformation complexes of morphisms (see remark 2.3.1), the analogously defined map $\phi_{\#}: C_{d e f}^{*}(\phi \circ \psi) \longrightarrow C_{d e f}^{*}(\phi)$ is an inverse of $\phi^{*}$.

\subsubsection{Deformation complex of Lie subgroupoids}

If $\mathcal{H} \stackrel{\iota}{\hookrightarrow} \mathcal{G}$ is a Lie subgroupoid, the deformation complex of the Lie subgroupoid $\mathcal{H} \subset \mathcal{G}$, $C_{\text {def }}^{*}(\mathcal{H} \subset \mathcal{G})$, is defined as the quotient complex induced by the injection $C_{\text {def }}^{*}(\mathcal{H}) \stackrel{\iota_{*}^{*}}{\rightarrow} C_{\text {def }}^{*}(\iota)$. 
That is, we have the exact sequence of complexes

$$
0 \rightarrow C_{\text {def }}^{*}(\mathcal{H}) \stackrel{\iota_{*}}{\longrightarrow} C_{\text {def }}^{*}(\iota) \longrightarrow C_{\text {def }}^{*}(\mathcal{H} \subset \mathcal{G}) \rightarrow 0,
$$

where the injectivity of $i_{*}$ is implied by the injectivity of $d_{h} \iota: T_{h} \mathcal{H} \longrightarrow T_{\iota(h)} \mathcal{G}$ for all $h \in \mathcal{H}$. Denote by $\bar{\delta}$ the differential of $C_{\text {def }}^{*}(\mathcal{H} \subset \mathcal{G})$ induced from the differential $\delta$ of $C_{\text {def }}^{*}(\iota)$.

We show now that these cohomologies have the expected role in the deformation theory of its respective structures.

\subsection{Infinitesimal cocycles associated to deformations}

In this section we explain the relation between deformations of morphisms and Lie subgroupoids and the corresponding deformation cohomologies. Such a connection, in the case of morphisms, is initially given by Proposition 2.4.1.

Let $\phi_{0}: \mathcal{H} \rightarrow \mathcal{G}$ be a Lie groupoid morphism. Assume that $\left\{\phi_{\epsilon}\right\}_{\epsilon \in I}$ is a deformation of $\phi_{0}$. We define the associated 1-cochain, $\tilde{X}_{0} \in C_{d e f}^{1}\left(\phi_{0}\right)$, by

$$
\tilde{X}_{0}(h):=\left.\frac{d}{d \epsilon}\right|_{\epsilon=0} \phi_{\epsilon}(h) \in T_{\phi_{0}(h)} \mathcal{G}
$$

Proposition 2.4.1 The 1-cochain $\tilde{X}_{0} \in C_{d e f}^{1}\left(\phi_{0}\right)$ in 2.3, is a 1-cocycle. The corresponding cohomology class in $H_{d e f}^{1}\left(\phi_{0}\right)$ depends only on the equivalence class of the deformation.

Proof. For the first part, it suffices to take derivatives at $\epsilon=0$ of the morphism condition satisfied by each $\phi_{\epsilon}, \phi_{\epsilon}\left(\bar{m}_{\mathcal{H}}(g h, h)\right)=\bar{m}_{\mathcal{G}}\left(\phi_{\epsilon}(g h), \phi_{\epsilon}(h)\right)$. In fact, we get

$$
\tilde{X}_{0}(g)-d \bar{m}_{\mathcal{G}}\left(\tilde{X}_{0}\left(m_{\mathcal{H}}(g, h)\right), \tilde{X}_{0}(h)\right)=0,
$$

which says that $\tilde{X}_{0}$ is a 1 -cocycle.

Take now $\left\{\psi_{\epsilon}=I_{\sigma(\epsilon)} \circ \phi_{\epsilon}\right\}$ an equivalent deformation of $\phi_{0}$ and denote $\tilde{X}_{0}^{\prime}$ the associated cocycle. The exactness of $\tilde{X}_{0}^{\prime}-\tilde{X}_{0}$ comes from taking derivatives at $\epsilon=0$ of the equivalence condition $\psi_{\epsilon}=I_{\sigma(\epsilon)} \circ \phi_{\epsilon}$. Thus, it suffices to use the chain rule and look at the expression $\left.\frac{d}{d \epsilon}\right|_{\epsilon=0} I_{\sigma_{\epsilon}}$. Therefore, the proof follows from the next lemma.

Due to the previous proposition, the element $\tilde{X}_{0}$ is also called the infinitesimal cocycle associated to the deformation $\left\{\phi_{\epsilon}\right\}_{\epsilon}$.

Lemma 2.4.2 If $\left\{\phi_{\epsilon}\right\}$ is a trivial deformation of $\left(\phi_{0}, \bar{\phi}_{0}\right)$, its infinitesimal cocycle $\tilde{X}_{0}$ is exact.

Proof. Assume $\phi_{\epsilon}=I_{\sigma_{\epsilon}} \circ \phi_{0}$, for $\sigma_{\epsilon}$ a smooth family of bisections of $\mathcal{G}$ with $\sigma_{0}=1_{\mathcal{G}}$. Then, 


$$
\begin{aligned}
\left.\frac{d}{d \epsilon}\right|_{\epsilon=\lambda}\left(I_{\sigma_{\epsilon}} \circ \phi_{0}\right)(h) & =\left.\frac{d}{d \epsilon}\right|_{\epsilon=\lambda} m\left(\sigma_{\epsilon}\left(t\left(\phi_{0}(h)\right)\right) \cdot \phi_{0}(h), \sigma_{\lambda}\left(s\left(\phi_{0}(h)\right)\right)^{-1}\right) \\
& +\left.\frac{d}{d \epsilon}\right|_{\epsilon=\lambda} m\left(\sigma_{\lambda}\left(t\left(\phi_{0}(h)\right)\right) \cdot \phi_{0}(h), \sigma_{\epsilon}\left(s\left(\phi_{0}(h)\right)\right)^{-1}\right) \\
& =\left.\frac{d}{d \epsilon}\right|_{\epsilon=\lambda} R_{\phi_{0}(h) \sigma_{\lambda}\left(s\left(\phi_{0}(h)\right)\right)^{-1}}\left(\sigma_{\epsilon}\left(\overline{\phi_{0}}(t(h))\right)\right) \\
& +\left.\frac{d}{d \epsilon}\right|_{\epsilon=\lambda} L_{\sigma_{\lambda}\left(\overline{\phi_{0}}(t(h))\right) \phi_{0}(h)}\left(\sigma_{\epsilon}\left(\overline{\phi_{0}}(s(h))\right)^{-1}\right) \\
& =r_{I_{\sigma_{\lambda}} \circ \phi_{0}}(h)\left(\left.\frac{d}{d \epsilon}\right|_{\epsilon=\lambda}\left(\sigma_{\epsilon}\left(\overline{\phi_{0}}(t(h))\right) \cdot \sigma_{\lambda}\left(\overline{\phi_{0}}(s(h))\right)^{-1}\right)\right) \\
& +l_{I_{\sigma_{\lambda}} \circ \phi_{0}(h)}\left[d i\left(\left.\frac{d}{d \epsilon}\right|_{\epsilon=\lambda}\left(\sigma_{\epsilon}\left(\overline{\phi_{0}}(t(h))\right) \cdot \sigma_{\lambda}\left(\bar{\phi}_{0}(s(h))\right)^{-1}\right)\right)\right] .
\end{aligned}
$$

Define then the family of sections $\left\{\alpha^{\lambda} \in \Gamma\left(A_{\mathcal{G}}\right)\right\}_{\lambda}$ by

$$
\alpha_{\varphi_{\lambda}(x)}^{\lambda}:=\left.\frac{d}{d \epsilon}\right|_{\epsilon=\lambda}\left(\sigma_{\epsilon}(x) \sigma_{\lambda}(x)^{-1}\right), \text { for each } \lambda,
$$

where $\varphi_{\lambda}$ is the map on the unit space of $\mathcal{G}$ associated to the automorphism $I_{\sigma_{\lambda}}$. Thus, by taking the family of pullback sections, $\bar{\alpha}_{\epsilon}:=\left(\varphi_{\epsilon} \circ \phi_{0}\right)^{*} \alpha^{\epsilon} \in C_{d e f}^{0}\left(I_{\sigma_{\epsilon}} \circ \phi_{0}=\phi_{\epsilon}\right)$ we get

$$
\left.\frac{d}{d \epsilon}\right|_{\epsilon=\lambda}\left(I_{\sigma_{\epsilon}} \circ \phi_{0}\right)(h)=\delta_{I_{\sigma_{\lambda}} \circ \phi_{0}}\left(\bar{\alpha}_{l}\right)(h) .
$$

Take now $\lambda=0$ to finish the proof.

Remark 2.4.3 Note that since a deformation $\left\{\phi_{\epsilon}\right\}$ of $\phi_{0}$ can be seen as a deformation of $\phi_{\lambda}$ for any $\lambda \in I$, the corresponding $\tilde{X}_{\lambda} \in C_{\text {def }}^{1}\left(\phi_{\lambda}\right)$ is also a cocycle. Thus, the previous proof shows even more, namely, that in a trivial deformation every $\tilde{X}_{\lambda}$ is exact. However, this fact also follows from a simple observation: if $\left\{\phi_{\epsilon}\right\}$ is a trivial deformation of $\phi_{0},\left\{\phi_{\epsilon}\right\}$ also can be regarded as a trivial deformation of $\phi_{\lambda}$ for any $\lambda \in I$; namely, $\phi_{\epsilon}=I_{\sigma_{\epsilon} \star \sigma_{\lambda}^{-1}} \circ \phi_{\lambda}$, where $\star$ is the product in the group of bisections of $\mathcal{G}$, given by $(\sigma \star \tau)(x)=\sigma(t(\tau(x))) \tau(x)$; for $x \in M$, and $\sigma$ and $\tau$ bisections of $\mathcal{G}$. Hence, in this case, every associated cocycle $\tilde{X}_{\lambda}$ is also exact.

Similarly, we can study analogue results by considering deformations of Lie subgroupoids. Just as in the situation described in Subsection 2.1.1 for deformations of Lie groupoids, it turns out that $s$-constant deformations of Lie subgroupoids have a more direct and explicit relation with their corresponding deformation complexes. Due to that, below we will work with $s$-constant deformations, exhibiting such a direct approach for them, and the general case of non necessarily $s$-constant deformations will be considered in Section 2.8.

Let $\left\{\mathcal{H}_{\epsilon}\right\}_{\phi}$ be an $s$-constant deformation of $\mathcal{H} \subset \mathcal{G}$. We define the associated 1-cochain, $X_{0} \in C_{\text {def }}^{1}\left(\mathcal{H}_{0} \subset \mathcal{G}\right)$, by $X_{0}:=\left[\bar{X}_{0}\right]$ (the class of $\bar{X}_{0}$ in the quotient space $C_{\text {def }}^{1}(\mathcal{H} \subset \mathcal{G})=$ 
$\left.\frac{C_{d e f}^{1}(\iota)}{\iota_{*} C_{d e f}^{1}(\mathcal{H})}\right)$, where

$$
\bar{X}_{0}(h):=\left.\frac{d}{d \epsilon}\right|_{\epsilon=0} \phi_{\epsilon}(h) \in T_{\iota(h)} \mathcal{G}, \quad\left(\bar{X}_{0} \in C_{d e f}^{1}(\iota)\right) .
$$

The element $X_{0}$ is called the infinitesimal cocycle associated to the deformation $\left\{\mathcal{H}_{\epsilon}\right\}_{\phi}$ of $\mathcal{H} \subset \mathcal{G}$. This terminology is justified by the following

Proposition 2.4.4 The 1-cochain $X_{0} \in C_{d e f}^{1}(\mathcal{H} \subset \mathcal{G})$, defined above, is a 1-cocycle. The corresponding cohomology class in $H_{\text {def }}^{1}(\mathcal{H} \subset \mathcal{G})$ depends only on the equivalence class of the deformation.

Proof. For some $\lambda$ fixed, consider the compatibility condition satisfied by each $\phi_{\epsilon}$ of $\left\{\mathcal{H}_{\epsilon}\right\}_{\phi_{\epsilon}}$,

$$
\phi_{\epsilon}\left(\bar{m}_{\epsilon}\left(m_{\lambda}(h, k), k\right)\right)=\bar{m}_{\mathcal{G}}\left(\phi_{\epsilon}\left(m_{\lambda}(h, k)\right), \phi_{\epsilon}(k)\right),
$$

where $\bar{m}_{\epsilon}$ and $m_{\epsilon}$ denote the division and multiplication maps on $\mathcal{H}_{\epsilon}$, respectively. Note that in this expression it is fundamental the fact of taking an $s$-constant deformation. By applying $\left.\frac{d}{d \epsilon}\right|_{\epsilon=\lambda}$, we get

$$
\begin{aligned}
-d \bar{m}_{\mathcal{G}}\left(\bar{X}_{\lambda}\left(m_{\lambda}(h, k)\right), \bar{X}_{\lambda}(k)\right)+\bar{X}_{\lambda}(h) & =-d \phi_{\lambda}\left(\xi_{\lambda}(h, k)\right) \\
& =-\left[\left(\phi_{\lambda}\right)_{*} \xi_{\lambda}\right](h, k) .
\end{aligned}
$$

That is,

$$
\delta_{\phi_{\lambda}}\left(\bar{X}_{\lambda}\right)=-\left(\phi_{\lambda}\right)_{*} \xi_{\lambda} \in\left(\phi_{\lambda}\right)_{*} C_{d e f}^{1}\left(\tilde{\mathcal{H}}_{\lambda}\right)
$$

Thus,

$$
\bar{\delta}_{\phi_{\lambda}}\left(X_{\lambda}\right)=0,
$$

and hence $\lambda=0$ gives rise to the fact that $X_{0}$ is a 1-cocycle of $C_{\text {def }}^{1}\left(\mathcal{H}_{0} \subset \mathcal{G}\right)$.

Take now $\left\{\mathcal{H}_{\epsilon}^{\prime}\right\}_{\psi_{\epsilon}}$ a deformation of $\mathcal{H} \subset \mathcal{G}$ equivalent to $\left\{\mathcal{H}_{\epsilon}\right\}_{\phi_{\epsilon}}$, and denote by $X_{0}^{\prime}$ its infinitesimal cocycle. By remark 2.2.8, this amounts to have $\phi_{\epsilon} \circ F_{\epsilon}=I_{\sigma_{\epsilon}} \circ \psi_{\epsilon}$; for some unique smooth families of isomorphisms $F_{\epsilon}: \tilde{\mathcal{H}}_{\epsilon}^{\prime} \longrightarrow \tilde{\mathcal{H}}_{\epsilon}$, and of bisections of $\mathcal{G},\left\{\sigma_{\epsilon}\right\}_{\epsilon}$, with $F_{0}=I d_{\mathcal{H}}$ and $\sigma_{0}=1_{\mathcal{G}}$. The exactness of $X_{0}-X_{0}^{\prime}$ comes from differentiatin at $\epsilon=0$ of such an equivalence condition $\phi_{\epsilon} \circ F_{\epsilon}=I_{\sigma_{\epsilon}} \circ \psi_{\epsilon}$. Thus, it suffices to use the chain rule and to look at the trivial deformation. Therefore, the proof follows from the following lemma, which shows that a trivial deformation has an exact infinitesimal cocycle.

Lemma 2.4.5 If $\left\{\mathcal{H}_{\epsilon}\right\}_{\left\{\phi_{\epsilon}\right\}}$ is a trivial deformation of $\mathcal{H} \subset \mathcal{G}$, its infinitesimal cocycle $X_{0}$ is exact.

Proof. By remark 2.2.8, $\left\{\mathcal{H}_{\epsilon}\right\}_{\phi}$ being a trivial deformation means that

$$
\phi_{\epsilon} \circ F_{\epsilon}=I_{\sigma_{\epsilon}} \circ \iota
$$


for families $F_{\epsilon}: \mathcal{H} \longrightarrow \tilde{\mathcal{H}}_{\epsilon}$ and $\sigma_{\epsilon} \in \operatorname{Bis}(\mathcal{G})$ with $F_{0}=I d_{\mathcal{H}}$ and $\sigma_{0}=1_{\mathcal{G}}$. In this way, by taking derivative with respect to $\epsilon$, we see that

$$
\begin{aligned}
\tilde{X}_{\lambda}\left(F_{\lambda}(h)\right)+d \phi_{\lambda}(\underbrace{\left.\left.\frac{d}{d \epsilon}\right|_{\epsilon=\lambda} F_{\epsilon}(h)\right)}_{=: \hat{T}_{\lambda}} & =\left.\frac{d}{d \epsilon}\right|_{\epsilon=\lambda} I_{\sigma_{\epsilon}} \circ \iota(h) \\
& \left.=\delta_{I_{\sigma_{\lambda}} \iota}\left(\bar{\alpha}^{\lambda}\right)_{(h)} \quad \text { (Proposition 2.4.2 with } \phi_{0}=\iota\right) .
\end{aligned}
$$

In other words, we have

$$
\begin{aligned}
& \tilde{X}_{\lambda}\left(F_{\lambda}(h)\right)=\delta_{I_{\sigma_{\lambda}} \circ \iota}\left(\bar{\alpha}^{\lambda}\right)_{(h)}-\left(\left(\phi_{\lambda}\right)_{*} \hat{T}_{\lambda}\right)_{(h)} ; \quad \hat{T}_{\lambda} \in C_{d e f}^{1}\left(F_{\lambda}\right) \\
& =\delta_{I_{\sigma_{\lambda} \circ \iota}}\left(\bar{\alpha}^{\lambda}\right)_{(h)}-\left(\left(\phi_{\lambda}\right)_{*}\left(F_{\lambda}^{*} T_{\lambda}\right)\right)_{(h)} ; \quad T_{\lambda} \in C_{d e f}^{1}\left(\tilde{\mathcal{H}}_{\lambda}\right) \\
& =\delta_{\phi_{\lambda} \circ F_{\lambda}}\left(\bar{\alpha}^{\lambda}\right)_{(h)}-\left(F_{\lambda}^{*}\left(\phi_{\lambda}\right)_{*} T_{\lambda}\right)_{(h)} \\
& =\left(F_{\lambda}^{*} \circ \delta_{\phi_{\lambda}} \circ\left(F_{\lambda}\right)_{\#}\right)\left(\bar{\alpha}^{\lambda}\right)_{(h)}-\left(F_{\lambda}^{*}\left(\phi_{\lambda}\right)_{*} T_{\lambda}\right)_{(h)},
\end{aligned}
$$

where in the last equality we use that $F_{\lambda}^{*}$ and $\left(F_{\lambda}\right)_{\#}$ are cochain maps.

Thus, by applying $\left(F_{\lambda}\right)_{\#}$, we get

$$
\tilde{X}_{\lambda}(h)=\delta_{\phi_{\lambda}}\left(\tilde{\alpha}^{\lambda}\right)_{(h)}-\left(\left(\phi_{\lambda}\right)_{*} T_{\lambda}\right)_{(h)} ; \tilde{\alpha}^{\lambda}:=\left(F_{\lambda}\right)_{\#}\left(\bar{\alpha}^{\lambda}\right) \in C_{d e f}^{0}\left(\phi_{\lambda}\right) .
$$

That is, the 1-cocycle $X_{\lambda}=\left[\tilde{X}_{\lambda}\right] \in C_{d e f}^{1}\left(\mathcal{H}_{\lambda} \subset \mathcal{G}\right)$ is exact: $X_{\lambda}=\left[\delta_{\phi_{\lambda}}\left(\tilde{\alpha}^{\lambda}\right)\right]=\bar{\delta}_{\phi_{\lambda}}\left[\tilde{\alpha}^{\lambda}\right]$.

\subsection{Moser's trick}

In this section we describe Moser's deformation argument in the context of morphisms of Lie groupoids and Lie subgroupoids. We begin with morphisms. First, a technical remark.

Remark 2.5.1 Note that, in the context of Lie groups, if $\phi_{\epsilon}: H \longrightarrow G$ is a family of morphisms of Lie groups, one has $C_{d e f}^{0}\left(\phi_{\epsilon}\right)=\mathfrak{g}$ for all $\epsilon \in I$, so talking about the smoothness of the family of 0-cochains $\left\{u_{\epsilon} \in C_{d e f}^{0}\left(\phi_{\epsilon}\right)\right\}_{\epsilon}$ simply amounts to talking of the smoothness of a family of elements in $\mathfrak{g}$. However, the situation in the context of Lie groupoids is a bit different. If $\left\{\left(\phi_{\epsilon}, \bar{\phi}_{\epsilon}\right): \mathcal{H} \longrightarrow \mathcal{G}\right\}_{\epsilon}$ is a smooth family of morphism of Lie groupoids, $C_{\text {def }}^{0}\left(\phi_{\epsilon}\right)=\Gamma_{N}\left(\bar{\phi}_{\epsilon}^{*} A_{\mathcal{G}}\right)$, where $N$ is the unit space of $\mathcal{H}$; so the space of 0 -degree cochains depends on the base map of the morphism. In this sense, we will say that a family of 0-cochains $\left\{\bar{\alpha}^{\epsilon} \in C_{\text {def }}^{0}\left(\phi_{\epsilon}\right)\right\}_{\epsilon}$ is smooth if the section $\bar{\alpha} \in \Gamma_{N \times I}\left(\bar{\phi}^{*} A_{\tilde{\mathcal{G}}}\right)$ defined by $\bar{\alpha}(x, \epsilon):=\left(\bar{\alpha}^{\epsilon}(x), \epsilon\right)$ is smooth; where $\tilde{\mathcal{G}}=\mathcal{G} \times I$ and $(\phi, \bar{\phi})$ is the morphism between the groupoids $\tilde{\mathcal{H}} \rightrightarrows N \times I$ and $\tilde{\mathcal{G}} \rightrightarrows M \times I$ such that restricted to the fiber over $\epsilon$ is $\left(\phi_{\epsilon}, \bar{\phi}_{\epsilon}\right)$.

Theorem 2.5.2 Let $\left(\phi_{0}, \bar{\phi}_{0}\right):(\mathcal{H} \rightrightarrows N) \rightarrow(\mathcal{G} \rightrightarrows M)$ be a morphism of Lie groupoids. Assume that $\bar{\phi}_{0}$ is an injective imersion and $N$ is compact. If the associated cocycle $X_{\epsilon}=\frac{d}{d \epsilon} \phi_{\epsilon}$ 
is exact in a smooth manner, then the deformation $\left\{\phi_{\epsilon}\right\}$ is trivial.

Remark 2.5.3 The smooth exactness condition of the 1-cocycles $X_{\epsilon}$ is just another way to say that $X_{\epsilon}=\delta_{\phi_{\epsilon}}\left(\bar{\alpha}^{\epsilon}\right)$, where $\bar{\alpha}^{\epsilon}$ is a smooth family of 0-cochains in the sense of Remark 2.5.1.

Proof. For this proof we use the notations of Remark 2.5.1 above. On the one hand, note that $\bar{\phi}(N \times I)$ is a closed embedded submanifold of $M \times I$. In fact, since $N$ is compact $\bar{\phi}_{0}$ is an embedding, thus, after shrinking $I$ if necessary, the map $\bar{\phi}: N \times I \longrightarrow M \times I$ is an injective imersion, and also an embedding due to the fact that each $\bar{\phi}_{\epsilon}$ is an embedding. Moreover, the compactness of $N$ also implies that $\bar{\phi}(N \times I)$ is closed inside $M \times I$. On the other hand, since $\bar{\phi}$ is injective then the section $\bar{\alpha} \in \Gamma\left(\bar{\phi}^{*} A_{\tilde{\mathcal{G}}}\right)$ can be regarded as a section of $\left.A_{\tilde{\mathcal{G}}}\right|_{\bar{\phi}(N \times I)}$. Therefore, the section $\bar{\alpha}$ can be extended to a section $\alpha$ of all $A_{\mathcal{G} \times I}$. Choose the extension in such a way that its support is contained in an open subset $U \times I \subset M \times I$, where $U \subset M$ is an open subset containing $\bar{\phi}_{0}(N)$ with compact closure $\bar{U}$. Note that this extended section has the form $\alpha(x, \epsilon)=\alpha^{\epsilon}(x)$, with $\alpha^{\epsilon} \in \Gamma\left(A_{\mathcal{G}}\right)$ extending $\bar{\alpha}^{\epsilon} \in \Gamma\left(\bar{\phi}_{\epsilon}^{*} A_{\mathcal{G}}\right)$.

In this way, each $X_{\epsilon}=\delta_{\phi_{\epsilon}}\left(\bar{\alpha}^{\epsilon}\right)$ is the restriction of the vector field $Z_{\epsilon}:=\delta_{\mathcal{G}}\left(\alpha^{\epsilon}\right) \in C_{\text {def }}^{1}(\mathcal{G})$ to $\phi_{\epsilon}(\mathcal{H}) \subset \mathcal{G}$. In fact,

$$
\begin{aligned}
X_{\epsilon}(h)=\delta_{\phi_{\epsilon}}\left(\bar{\alpha}^{\epsilon}\right)_{(h)} & =r_{\phi_{\epsilon}(h)}\left(\bar{\alpha}^{\epsilon}(t(h))\right)+l_{\phi_{\epsilon}(h)}\left(d i\left(\bar{\alpha}^{\epsilon}(s(h))\right)\right) \\
& =r_{\phi_{\epsilon}(h)}\left(\alpha^{\epsilon}\left(\bar{\phi}_{\epsilon}(t(h))\right)\right)+l_{\phi_{\epsilon}(h)}\left(\operatorname{di}\left(\alpha^{\epsilon}\left(\bar{\phi}_{\epsilon}(s(h))\right)\right)\right) \\
& =r_{\phi_{\epsilon}(h)}\left(\alpha^{\epsilon}\left(t \circ \phi_{\epsilon}(h)\right)\right)+l_{\phi_{\epsilon}(h)}\left(\operatorname{di}\left(\alpha^{\epsilon}\left(s \circ \phi_{\epsilon}(h)\right)\right)\right) \\
& =\delta_{\mathcal{G}}\left(\alpha^{\epsilon}\right)_{\left(\phi_{\epsilon}(h)\right)} .
\end{aligned}
$$

Now, consider the time-dependent vector field $\vec{\alpha}:=\left\{\vec{\alpha}^{\epsilon}\right\}$ on $\mathcal{G}$, and denote by $\psi^{t_{1}, t_{0}}$ its (time-dependent) flow. Due to the compactness of $\bar{U}$ and the vanishing of the sections outside $U$, the flow $\psi^{\epsilon, 0}$ is defined on all $M$ for $\epsilon$ samll enough. Let $\sigma_{\epsilon}$ be the family of bisections of $\mathcal{G}$ given by, by $\sigma_{\epsilon}(x):=\psi^{\epsilon, 0}(x)$ for $x \in M$, and consider the associated deformation $\left\{I_{\sigma_{\epsilon}} \circ \phi_{0}\right\}_{\epsilon}$ of $\phi_{0}$. We will show now that $\phi_{\epsilon}=I_{\sigma_{\epsilon}} \circ \phi_{0}$, for $\epsilon$ small enough, which will completes the proof.

In fact, by Lemma 2.4.2, the deformation $\left\{I_{\sigma_{\epsilon}} \circ \phi_{0}\right\}_{\epsilon}$ has exact associated cocycles. Namely,

$$
\begin{aligned}
Y_{\lambda}(h) & :=\left.\frac{d}{d \epsilon}\right|_{\epsilon=\lambda} I_{\sigma_{\epsilon}}\left(\phi_{0}(h)\right) \\
& =r_{I_{\sigma_{\lambda}}\left(\phi_{0}(h)\right)}\left(\alpha_{\varphi_{\lambda}\left(t\left(\phi_{0}(h)\right)\right)}^{\lambda}\right)+l_{I_{\sigma_{\lambda}}\left(\phi_{0}(h)\right)}\left(\operatorname{di}\left(\alpha_{\varphi_{\lambda}\left(s\left(\phi_{0}(h)\right)\right)}^{\lambda}\right)\right) \\
& =\delta_{I_{\sigma_{\lambda}} \circ \phi_{0}}\left(\hat{\alpha}^{\lambda}\right)_{(h)},
\end{aligned}
$$


where $\hat{\alpha}^{\lambda}=\left(\varphi_{\lambda} \circ \bar{\phi}_{0}\right)^{*} \alpha^{\lambda} \in \Gamma_{N}\left(\left(\varphi_{\lambda} \circ \bar{\phi}_{0}\right)^{*} A_{\mathcal{G}}\right)$ and, as before, $\varphi_{\lambda}$ is the base map associated to $I_{\sigma_{\lambda}}$. Hence, $Y_{\lambda}$ is the restriction of $\delta_{\mathcal{G}}\left(\alpha^{\lambda}\right)$ to $\left(I_{\sigma_{\lambda}} \circ \phi_{0}\right)(\mathcal{H})$ :

$$
\delta_{\mathcal{G}}\left(\alpha^{\lambda}\right)_{\left(I_{\sigma_{\lambda}} \circ \phi_{0}\right)(h)}=r_{I_{\sigma_{\lambda}}\left(\phi_{0}(h)\right)}\left(\alpha_{t\left(I_{\sigma_{\lambda}} \circ \phi_{0}(h)\right)}^{\lambda}\right)+l_{I_{\sigma_{\lambda}}\left(\phi_{0}(h)\right)}\left(d i\left(\alpha_{s\left(I_{\sigma_{\lambda}} \circ \phi_{0}(h)\right)}\right)\right) .
$$

Summarizing, by (2.5), $X_{\lambda}$ can be viewed as a restriction of $Z_{\lambda}$; and similarly, by (2.6), $Y_{\lambda}$ as a restriction of $Z_{\lambda}$. In other words, (2.5) and (2.6) tell us that $\left\{\epsilon \mapsto \phi_{\epsilon}(h)\right\}$ and $\left\{\epsilon \mapsto I_{\sigma_{\epsilon}} \circ \phi_{0}(h)\right\}$ are integral curves of the time-dependent vector field, $\left\{Z_{\epsilon}\right\}_{\epsilon}$, passing through $\phi_{0}(h) \in \mathcal{G}$ at time $\epsilon=0$. Therefore, $\phi_{\epsilon}(h)=I_{\sigma_{\epsilon}}(\phi(h))$ for all $\epsilon$ small enough.

Remark 2.5.4 In the previous proof we could have extended the section $\bar{\alpha}$ to another $\alpha^{\prime} \neq \alpha$, getting a family of bisections $\left\{\sigma_{\epsilon}^{\prime}\right\} \neq\left\{\sigma_{\epsilon}\right\}$. With this other family we would arrive at the same conclusion because they agree when restricted to $\bar{\phi}_{0}(N)$, i.e., $\sigma_{\epsilon}(x)=\sigma_{\epsilon}^{\prime}(x)$, for all $x \in \bar{\phi}_{0}(N)$. In fact, their values over $\bar{\phi}_{0}(N)$ are determined by the elements $\bar{\alpha}_{\epsilon}$, as expression 2.4 shows. Therefore, the proof does not depend on the choice of the extension $\alpha$.

For Lie subgroupoids we also have a result analogous to that of Theorem above. Before stating it we make a definition in the same spirit as remark 2.5.1.

Let $\left\{\mathcal{H}_{\epsilon}\right\}_{\left\{\phi_{\epsilon}\right\}}$ be a deformation of $\mathcal{H} \subset \mathcal{G}$. Note that, by definition, the subgroupoid deformation complex in degree zero for each $\mathcal{H}_{\epsilon} \subset \mathcal{G}$ is $C_{\text {def }}^{0}\left(\mathcal{H}_{\epsilon} \subset \mathcal{G}\right)=\frac{\Gamma_{N}\left(\varphi_{\epsilon}^{*} A_{\mathcal{G}}\right)}{\Gamma_{N}\left(\varphi_{\epsilon}^{*} A_{\mathcal{H}}\right)}$. In this way,

Remark 2.5.5 Assume $\left\{\left[\tilde{\alpha}^{\epsilon}\right]\right\}_{\epsilon \in I}$ is a family of 0-cochains, $\left[\tilde{\alpha}^{\epsilon}\right] \in C_{\text {def }}^{0}\left(\mathcal{H}_{\epsilon} \subset \mathcal{G}\right)$. We say that $\left\{\left[\tilde{\alpha}^{\epsilon}\right]\right\}_{\epsilon \in I}$ is a smooth family if there exist representing $\hat{\alpha}_{\epsilon} \in\left[\tilde{\alpha}^{\epsilon}\right]$ such that $\left\{\hat{\alpha}_{\epsilon}\right\}_{\epsilon}$ is a smooth family of cochains in the sense of remark 2.5.1.

Theorem 2.5.6 Let $\left\{\tilde{\mathcal{H}}_{\epsilon}\right\}_{\epsilon} \stackrel{\left\{\phi_{\epsilon}\right\}}{\rightarrow} \mathcal{G}$ be a s-constant deformation of $\mathcal{H} \subset \mathcal{G}$, with $\mathcal{H}$ compact. If each associated cocycle $X_{\epsilon}=\left[\tilde{X}_{\epsilon}\right]$ is exact and equal to $\bar{\delta}_{\phi_{\epsilon}}\left(\left[\tilde{\alpha}^{\epsilon}\right]\right)$, with $\left\{\left[\tilde{\alpha}^{\epsilon}\right]\right\}_{\epsilon}$ a smooth family of cochains, then $\left\{\mathcal{H}_{\epsilon}\right\}_{\epsilon}$ is trivial.

Proof. If $X_{\epsilon}=\left[\tilde{X}_{\epsilon}\right]=\bar{\delta}_{\phi_{\epsilon}}\left(\left[\tilde{\alpha}^{\epsilon}\right]\right)$ in a smooth way, by the remark above, we have

$$
\tilde{X}_{\lambda}(h)=\delta_{\phi_{\lambda}}\left(\tilde{\alpha}^{\lambda}\right)_{(h)}-\left(\left(\phi_{\lambda}\right)_{*} T_{\lambda}\right)_{(h)} \text {, for some } T_{\lambda} \in C_{d e f}^{1}\left(\tilde{\mathcal{H}}_{\lambda}\right) \text {, for any } \lambda \text {. }
$$

Moreover, smoothness of $\left\{\tilde{\alpha}_{\epsilon}\right\}_{\epsilon}$ implies smoothness of the time-dependent vector field $T:=$ $\left\{T_{\epsilon}\right\}_{\epsilon}$ defined on $\tilde{\mathcal{H}}_{0}=\mathcal{H}$. Then, by applying $\delta_{\phi_{\lambda}}$ to the expression above, we get

$$
-\left(\phi_{\lambda}\right)_{*} \xi_{\lambda}=-\left(\phi_{\lambda}\right)_{*}\left(\delta_{\tilde{\mathcal{H}}_{\lambda}} T_{\lambda}\right)
$$

Or equivalently, by injectivity of $\left(\phi_{\lambda}\right)_{*}$,

$$
\delta_{\lambda} T_{\lambda}=\xi_{\lambda} ;
$$


which says that the (time-dependent) flow of $T=\left\{T_{\epsilon}\right\}_{\epsilon}$ gives rise to Lie groupoid isomorphisms $F_{\epsilon}: \tilde{\mathcal{H}}_{0} \longrightarrow \tilde{\mathcal{H}}_{\epsilon}, \epsilon \in J \subset I$ (Proposition 2.1.6).

Now, define $\psi_{\epsilon}:=\phi_{\epsilon} \circ F_{\epsilon}$. We will show that $\left\{\left(\tilde{\mathcal{H}}_{0}, \psi_{\epsilon}\right)\right\}_{\epsilon \in J}$ is a trivial deformation of $\mathcal{H} \subset \mathcal{G}$, completing the proof (the family $\left\{F_{\epsilon}\right\}_{\epsilon}$ will give us the equivalence between $\left\{\mathcal{H}_{\epsilon}\right\}_{\psi_{\epsilon}}$ and $\left.\left\{\mathcal{H}_{\epsilon}\right\}_{\phi_{\epsilon}}\right)$.

In fact, note that $\left\{\psi_{\epsilon}\right\}_{\epsilon}$ is a deformation of the inclusion morphism $\iota: \mathcal{H} \longrightarrow \mathcal{G}$, and looking at the associated cocycles, $\tilde{X}_{\lambda}^{\prime} \in C_{d e f}^{1}\left(\psi_{\lambda}\right)$, we have

$$
\begin{aligned}
\tilde{X}_{\lambda}^{\prime}(h) & =\tilde{X}_{\lambda}\left(F_{\lambda}(h)\right)+\left(\left(\phi_{\lambda}\right)_{*} T_{\lambda}\right)_{\left(F_{\lambda}(h)\right)} \\
& =\delta_{\phi_{\lambda}}\left(\tilde{\alpha}^{\lambda}\right)_{\left(F_{\lambda}(h)\right)} \\
& =\delta_{\phi_{\lambda} \circ F_{\lambda}}\left(\bar{\alpha}^{\lambda}\right)_{(h)}=\delta_{\psi_{\lambda}}\left(\bar{\alpha}^{\lambda}\right)_{(h)}, \text { where } \bar{\alpha}^{\lambda}:=F_{\lambda}^{*} \tilde{\alpha}^{\lambda} \in C_{d e f}^{0}\left(\psi_{\lambda}\right) .
\end{aligned}
$$

Hence, by Theorem 2.5.2, we get $\psi_{\epsilon}=I_{\sigma_{\epsilon}} \circ \iota$, that is, $\left\{\psi_{\epsilon}\right\}_{\epsilon}$ is trivial.

Looking again to examples 2.2.6, note that another point of view of triviality of deformations can be considered. For instance, a family of elements in $A u t(\mathcal{G})$ determines a deformation of a given morphism $\phi_{0}: \mathcal{H} \longrightarrow \mathcal{G}$ and a Lie subgroupoid $\mathcal{H} \subset \mathcal{G}$. In this direction, we want now to adapt the main results above to the context of all automorphisms of $\mathcal{G}$. That is, to use $A u t(\mathcal{G})$ to define trivial deformations, instead of just the subgroup of inner automorphisms. In this sense, a trivial deformation of $\phi_{0}$ will be a deformation of the form $\left\{\phi_{\epsilon}=F_{\epsilon} \circ \phi_{0}\right\}_{\epsilon}$, where $\left\{F_{\epsilon}\right\}_{\epsilon}$ is a smooth family of elements in $A u t(\mathcal{G})$, with $F_{0}=I d_{\mathcal{G}}$; and similarly we define trivial deformations of Lie subgroupoids.

For the following result we use the notations of remark 2.2.2.

Proposition 2.5.7 Let $(\phi, \bar{\phi})$ be a deformation of $\left(\phi_{0}, \bar{\phi}_{0}\right)$, with $\bar{\phi}$ injective and $\mathcal{G}$ compact. If the map in cohomology $\phi^{*}: H_{\text {def }}^{1}(\mathcal{G} \times I) \rightarrow H_{\text {def }}^{1}(\phi)$ is surjective, the deformation $\phi$ is trivial with respect to $\operatorname{Aut}(\mathcal{G})$.

Proof. Since $\phi^{*}$ is surjective, there exist a cocycle $\hat{Z} \in C_{\text {def }}^{1}(\mathcal{G} \times I)\left(=\mathfrak{X}_{s \times p r_{I}-\operatorname{proj}}(\mathcal{G} \times I)\right)$ and a cochain $\bar{\alpha} \in C_{\text {def }}^{0}(\phi)$ such that $\tilde{X}-\phi^{*}(\hat{Z})=\delta_{\phi}(\bar{\alpha})$. Then, by extending $\bar{\alpha}$ to a section of $A_{\mathcal{G} \times I}$, there exists $\bar{Z} \in C_{\text {def }}^{1}(\mathcal{G} \times I)_{c l}$ (i.e. $\bar{Z}(g, \epsilon)=\left(Z_{\epsilon}(g), f(s(g), \epsilon)\right)$ for some smooth family $\left\{Z_{\epsilon}\right\}_{\epsilon} \subset C_{d e f}^{1}(\mathcal{G})_{c l}$, and some $\left.f \in C^{\infty}(M \times I)\right)$ which satisfies

$$
\frac{d}{d \epsilon} \phi_{\epsilon}(h)=\tilde{X}_{\epsilon}(h)=Z_{\epsilon}\left(\phi_{\epsilon}(h)\right)
$$

Hence, if $F_{\epsilon}:=\psi^{\epsilon, 0}$ are the automorphisms of $\mathcal{G}$ coming from the flow of the timedependent vector field on $\mathcal{G}, Z:=\left\{Z_{\epsilon}\right\}_{\epsilon}$, we get $F_{\epsilon}\left(\phi_{0}(h)\right)=\phi_{\epsilon}(h)$. That is, the deformation is trivial. 
A similar result for Lie subgroupoids also holds. Consider the projection between complexes $\pi: C_{d e f}^{*}(\phi) \rightarrow C_{d e f}^{*}(\phi(\mathcal{H} \times I) \subset \mathcal{G} \times I)$.

Theorem 2.5.8 Let $\left\{\mathcal{H}_{\epsilon}\right\}_{\left\{\phi_{\epsilon}\right\}}=\left\{\mathcal{H}_{\epsilon}\right\}_{\phi}$ be a s-constant deformation of $\mathcal{H} \subset \mathcal{G}$, with $\mathcal{H}$ and $\mathcal{G}$ compact. If the map $H_{\text {def }}^{1}(\mathcal{G} \times I) \stackrel{\pi \circ(\phi)^{*}}{\rightarrow} H_{\text {def }}^{1}(\phi(\mathcal{H} \times I) \subset \mathcal{G} \times I)$ is surjective, the deformation $\left\{\mathcal{H}_{\epsilon}\right\}_{\phi}$ is trivial with respect to $\operatorname{Aut}(\mathcal{G})$.

Proof. The proof follows an analogue idea to that of Theorem 2.5.6. Let $[\bar{X}] \in C_{\text {def }}^{1}(\phi(\mathcal{H} \times$ $I) \subset \mathcal{G} \times I)$ be the cocycle defined by the deformation $\left\{\mathcal{H}_{\epsilon}\right\}_{\phi}$, that is, $\bar{X}(g, \epsilon):=\left(\bar{X}_{\epsilon}(g), \epsilon\right)$ where $\left[\bar{X}_{\epsilon}\right] \in C_{d e f}^{1}\left(\mathcal{H}_{\epsilon} \subset \mathcal{G}\right)$ is the family of cocycles corresponding to the deformation. From surjectivity of $\pi \circ \phi^{*}$ it follows that there exists $\bar{Z} \in C_{d e f}^{1}(\mathcal{G} \times I)_{c l}, \bar{Z}(g, \epsilon)=\left(Z_{\epsilon}(g), f(s(g), \epsilon)\right)$ (note that $Z_{\epsilon} \in C_{d e f}^{1}\left(\mathcal{G}_{\epsilon}\right)_{c l}$ ), such that

$$
\left[(\phi)^{*} \bar{Z}\right]-[\bar{X}]=\bar{\delta}_{\phi}([\bar{\alpha}]) ; \bar{\alpha} \in C_{d e f}^{0}(\phi),
$$

i.e.

$$
(\phi)^{*} \bar{Z}-\bar{X}-(\phi)_{*} \bar{T}=\delta_{\phi}(\bar{\alpha}) ; \quad \bar{T} \in C_{d e f}^{1}(\tilde{\mathcal{H}}) \quad\left(\bar{T}(h, \epsilon)=\left(T_{\epsilon}(h), r(s(h), \epsilon)\right),\right.
$$

which implies,

$$
\left(\phi_{\epsilon}\right)^{*} Z_{\epsilon}-\bar{X}_{\epsilon}-\left(\phi_{\epsilon}\right)_{*} T_{\epsilon}=\delta_{\phi_{\epsilon}}\left(\bar{\alpha}^{\epsilon}\right), \forall \epsilon \in I
$$

where $\bar{\alpha}(x, \epsilon)=\left(\bar{\alpha}^{\epsilon}(x), \epsilon\right)$.

Then, as in proof of Theorem 2.5.6, by extending $\bar{\alpha}$ to a section $\alpha$ of $A_{\mathcal{G} \times I}, \alpha(x, \epsilon)=$ $\left(\alpha_{\epsilon}(x), \epsilon\right)$, we get that $\delta_{\phi_{\epsilon}}\left(\bar{\alpha}^{\epsilon}\right)$ can be viewed as the restriction of the field $W_{\epsilon}:=\delta\left(\alpha^{\epsilon}\right) \epsilon$ $C_{d e f}^{1}(\mathcal{G})_{c l}$.

In other words, equation (2.7) becomes

$$
\bar{X}_{\epsilon}=\left(\phi_{\epsilon}\right)^{*} \hat{Z}_{\epsilon}-\left(\phi_{\epsilon}\right)_{*} T_{\epsilon} ; \quad \hat{Z}_{\epsilon}:=Z_{\epsilon}-W_{\epsilon} \in C_{d e f}^{1}(\mathcal{G})_{c l}
$$

Applying $\delta_{\phi_{\epsilon}}$ to $(2.8)$

$$
-\left(\phi_{\epsilon}\right)_{*} \xi_{\epsilon}=\delta_{\phi_{\epsilon}} \bar{X}_{\epsilon}=-\left(\phi_{\epsilon}\right)_{*} \delta_{\epsilon} T_{\epsilon}
$$

Thus, by injectivity of $\left(\phi_{\epsilon}\right)_{*}$, the vector field $T \in \mathfrak{X}(\tilde{\mathcal{H}}), T(h, \epsilon)=\left(T_{\epsilon}(h), 0\right)+\left.\frac{\partial}{\partial \epsilon}\right|_{(h, \epsilon)}$, on $\tilde{\mathcal{H}}$ is multiplicative. Take $\left\{G_{\epsilon}: \tilde{\mathcal{H}}_{0} \rightarrow \tilde{\mathcal{H}}_{\epsilon}\right\}$ the family of isomorphisms induced by the flow of $T$, and define $\psi_{\epsilon}:=\phi_{\epsilon} \circ G_{\epsilon}$. We claim that $\left\{\left(\tilde{\mathcal{H}}_{0}, \psi_{\epsilon}\right)\right\}_{\epsilon}$ is a trivial deformation of $\mathcal{H} \subset \mathcal{G}$, which completes the proof. In fact, note that $\left\{\psi_{\epsilon}\right\}_{\epsilon}$ is a deformation of the inclusion morphism. Thus, looking at the associated cocycles $\tilde{X}_{\epsilon} \in C_{d e f}^{1}\left(\psi_{\epsilon}\right)$, we have

$$
\begin{aligned}
\tilde{X}_{\epsilon}(h):=\frac{d}{d \epsilon} \psi_{\epsilon}(h)=\bar{X}_{\epsilon}\left(G_{\epsilon}(h)\right)+\left[\left(\phi_{\epsilon}\right)_{*} T_{\epsilon}\right]_{\left(G_{\epsilon}(h)\right)} & =\left(\left(\phi_{\epsilon}\right)^{*} \hat{Z}_{\epsilon}\right)_{\left(G_{\epsilon}(h)\right)} \\
& =\hat{Z}_{\epsilon}\left(\psi_{\epsilon}(h)\right),
\end{aligned}
$$


where we use (2.8) in the third equality.

Therefore, from previous proposition, $\psi_{\epsilon}=F_{\epsilon} \circ \iota$, where $\left\{F_{\epsilon}\right\}$ are the automorphisms of $\mathcal{G}$ induced by the flow of the multiplicative vector field on $\mathcal{G},\left\{\hat{Z}_{\epsilon}\right\}_{\epsilon}$. Thus, the family $\left\{F_{\epsilon}\right\}_{\epsilon}$ gives us the equivalence between the deformations $\left\{\mathcal{H}_{\epsilon}\right\}_{\psi_{\epsilon}}$ and $\left\{\mathcal{H}_{\epsilon}\right\}_{\phi_{\epsilon}}$. That is, the deformation of $\mathcal{H} \subset \mathcal{G}$ given by $\left\{\left(\tilde{\mathcal{H}}_{\epsilon}, \phi_{\epsilon}\right)\right\}_{\epsilon}$ is trivial (w.r.t. $\operatorname{Aut}(\mathcal{G})$ ).

\subsection{Simultaneous deformations}

Following the spirit developed so far in studying deformations of morphisms and subgroupoids (definitions, deformation complex and Mosert's argument), we can now study the key facts of the most general case of deformations: given the triple of objects $\mathcal{H} \stackrel{\phi}{\longrightarrow} \mathcal{G}$, where $\phi$ is a morphism of groupoids, we want to deform each of the structures at the same time, keeping the compatibility between them. That is, we want a family of triples $\left\{\mathcal{H}_{\epsilon} \stackrel{\phi_{\epsilon}}{\longrightarrow} \mathcal{G}_{\epsilon}\right\}_{\epsilon \in I}$, where $\left\{\mathcal{H}_{\epsilon}\right\}_{\epsilon \in I}$ and $\left\{\mathcal{G}_{\epsilon}\right\}_{\epsilon \in I}$ are deformations of $\mathcal{H}$ and $\mathcal{G}$ respectively, and $\left\{\phi_{\epsilon}\right\}_{\epsilon \in I}$ is a smooth family of morphisms. In this way, we have the natural notion of equivalence of deformations.

Definition 2.6.1 (Equivalent deformations)

Let $\left\{\mathcal{H}_{\epsilon} \stackrel{\phi_{\epsilon}}{\longrightarrow} \mathcal{G}_{\epsilon}\right\}_{\epsilon}$ and $\left\{\mathcal{H}_{\epsilon}^{\prime} \stackrel{\psi_{\epsilon}}{\longrightarrow} \mathcal{G}_{\epsilon}^{\prime}\right\}_{\epsilon}$ be two deformations of the triple $\mathcal{H} \stackrel{\phi}{\longrightarrow} \mathcal{G}$. We say that they are equivalent if the deformations of $\mathcal{H}$ and $\mathcal{G}$ are equivalent in such a way that the corresponding deformations of morphisms are related by those equivalences, up to inner automorphisms. More explicitely, an equivalence is a family of triples $\left\{F_{\epsilon}, G_{\epsilon}, \sigma_{\epsilon}\right\}_{\epsilon \in I}$ where $\left\{F_{\epsilon}: \mathcal{H}_{\epsilon} \longrightarrow \mathcal{H}_{\epsilon}^{\prime}\right\}_{\epsilon}$ and $\left\{G_{\epsilon}: \mathcal{G}_{\epsilon} \longrightarrow \mathcal{G}_{\epsilon}^{\prime}\right\}_{\epsilon}$ are smooth families of isomorphisms and $\left\{\sigma_{\epsilon}\right.$ : $M \longrightarrow \mathcal{G}\}_{\epsilon \in I}$ is a smooth family of maps with $\sigma_{\epsilon}$ a bisection of the groupoid $\mathcal{G}_{\epsilon}$ for every $\epsilon$, such that they satify

$$
G_{\epsilon}^{-1} \circ \psi_{\epsilon} \circ F_{\epsilon}=I_{\sigma_{\epsilon}} \circ \phi_{\epsilon}
$$

for all $\epsilon$ small enough.

In this sense, a deformation $\left\{\mathcal{H}_{\epsilon} \stackrel{\phi_{\epsilon}}{\longrightarrow} \mathcal{G}_{\epsilon}\right\}_{\epsilon}$ of $\mathcal{H} \stackrel{\phi}{\longrightarrow} \mathcal{G}$ is said to be trivial if $I_{\sigma_{\epsilon}} \circ \phi=$ $G_{\epsilon}^{-1} \circ \phi_{\epsilon} \circ F_{\epsilon}$ for $\epsilon$ small enough, where $\left\{F_{\epsilon}: \mathcal{H} \longrightarrow \mathcal{H}_{\epsilon}\right\}_{\epsilon}$ and $\left\{G_{\epsilon}: \mathcal{G} \longrightarrow \mathcal{G}_{\epsilon}\right\}_{\epsilon}$ are families of isomorphisms of groupoids and $\sigma_{\epsilon}$ is a smooth family of bisections of $\mathcal{G}$.

\section{Deformation complex and Moser's trick}

Consider the diagram of cochain maps explained in Remark 2.3.1. Out of this data, we construct the complex which controls the deformations of the triple $\mathcal{H} \stackrel{\phi}{\longrightarrow} \mathcal{G}$ as follows. Take the mapping-cone complex associated to the cochain map $\phi_{*}$,

$$
M C^{*}\left(\phi_{*}\right)=C_{d e f}^{*+1}(\mathcal{H}) \oplus C_{d e f}^{*}(\phi)
$$

with differential $\delta_{M C\left(\phi_{*}\right)}(c, Y)=\left(\delta_{\mathcal{H}} c, \phi_{*} c-\delta_{\phi} Y\right)$. Note now that the map $\phi^{*}$ in Remark 2.3.1 induces a cochain map $\tilde{\phi}^{*}: C_{d e f}^{*}(\mathcal{G}) \longrightarrow M C^{*}\left(\phi_{*}\right)$ putting zero in the first compo- 
nent, $\tilde{\phi}^{*}: c \longmapsto\left(0,(-1)^{\operatorname{deg}(c)} c\right)$. Take now the mapping-cone associated to $\tilde{\phi}^{*}$, getting the deformation complex of the triple,

$$
\begin{aligned}
C_{\text {def }}^{*+1}(\mathcal{H}, \phi, \mathcal{G}) & :=M C^{*}\left(\tilde{\phi^{*}}\right)=M C^{*}\left(\phi_{*}\right) \oplus C_{\text {def }}^{*+1}(\mathcal{G}) \\
& =C_{\text {def }}^{*+1}(\mathcal{H}) \oplus C_{\text {def }}^{*}(\phi) \oplus C_{\text {def }}^{*+1}(\mathcal{G})
\end{aligned}
$$

with differential $\delta(c, X, \bar{c})=\left(-\delta_{\mathcal{H}} c, \delta_{\phi}(X)-\phi_{*} c+(-1)^{\operatorname{deg}(\bar{c})} \phi^{*} \bar{c}, \delta_{\mathcal{G}} \bar{c}\right)$.

In this way, given a deformation $\left\{\mathcal{H}_{\epsilon} \stackrel{\phi_{\epsilon}}{\longrightarrow} \mathcal{G}_{\epsilon}\right\}_{\epsilon}$ of $\{\mathcal{H} \stackrel{\phi}{\longrightarrow} \mathcal{G}\}$, by computations similar to those in Proposition 2.4.1 and Proposition 2.4.4, we get that $\left(\xi_{\mathcal{H}},-X, \xi_{\mathcal{G}}\right)$ is a 2-cocycle in $C_{\text {def }}^{*}(\mathcal{H}, \phi, \mathcal{G})$, where $\xi_{\mathcal{H}}$ and $\xi_{\mathcal{G}}$ are the respective deformation cocycles for $\mathcal{H}$ and $\mathcal{G}$, and $X=\left.\frac{d}{d \epsilon}\right|_{\epsilon=0} \phi_{\epsilon}$ is the usual cochain (Section 2.4) associated to a deformation of morphisms. In fact, that $\left(\xi_{\mathcal{H}},-X, \xi_{\mathcal{G}}\right)$ is a cocycle follows by applying $\left.\frac{d}{d \epsilon}\right|_{\epsilon=0}$ to the compatibility of $\left\{\mathcal{H}_{\epsilon} \stackrel{\phi_{\epsilon}}{\longrightarrow} \mathcal{G}_{\epsilon}\right\}_{\epsilon}$ :

$$
\begin{aligned}
& \left.\frac{d}{d \epsilon}\right|_{\epsilon=0} \phi_{\epsilon}\left(\bar{m}_{\mathcal{H}_{\epsilon}}(g h, h)\right)=\left.\frac{d}{d \epsilon}\right|_{\epsilon=0} \bar{m}_{\mathcal{G}_{\epsilon}}\left(\phi_{\epsilon}(g h), \phi_{\epsilon}(h)\right) \\
& \Longleftrightarrow \quad X(g)+\phi_{*} \xi_{\mathcal{H}}(g, h)=\xi_{\mathcal{G}}(\phi(g), \phi(h))+d \bar{m}_{\mathcal{G}}(X(g h), X(h)) \\
& \Longleftrightarrow \quad \delta_{\phi}(X)+\phi_{*} \xi_{\mathcal{H}}-\phi^{*} \xi_{\mathcal{G}}=0 .
\end{aligned}
$$

The fact that the corresponding cohomology class of $\left(\xi_{\mathcal{H}},-X, \xi_{\mathcal{G}}\right)$ only depends on the equivalence class of the deformation is also an analogous computation. Thus, we pass now to establish the main result concerning this type of most general deformations.

Theorem 2.6.2 Let $\left\{\mathcal{H}_{\epsilon} \stackrel{\left(\phi_{\epsilon}, \bar{\phi}_{\epsilon}\right)}{\longrightarrow} \mathcal{G}_{\epsilon}\right\}_{\epsilon}$ be a deformation of $(\mathcal{H} \stackrel{\phi}{\longrightarrow} \mathcal{G})$, with $\mathcal{H}$ and $\mathcal{G}$ compact, and $\bar{\phi}_{\epsilon}$ injective for every $\epsilon$. If the family of associated cocycles $\left\{\left(\xi_{\mathcal{H}_{\epsilon}},-X_{\epsilon}, \xi_{\mathcal{G}_{\epsilon}}\right) \in\right.$ $\left.C_{\text {def }}^{2}\left(\mathcal{H}_{\epsilon}, \phi_{\epsilon}, \mathcal{G}_{\epsilon}\right)\right\}_{\epsilon}$ is transgressed by a smooth family of cochains $\left\{\left(Y_{\epsilon}, \tilde{\alpha}_{\epsilon}, Z_{\epsilon}\right) \in C_{\text {def }}^{1}\left(\mathcal{H}_{\epsilon}, \phi_{\epsilon}, \mathcal{G}_{\epsilon}\right)\right\}_{\epsilon}$, then the deformation $\left\{\mathcal{H}_{\epsilon} \stackrel{\phi_{\epsilon}}{\longrightarrow} \mathcal{G}_{\epsilon}\right\}_{\epsilon}$ is trivial.

Proof. Exactness of the family of cocycles amounts to

$$
\begin{aligned}
\xi_{\mathcal{H}_{\epsilon}} & =-\delta_{\mathcal{H}_{\epsilon}} Y_{\epsilon} ; \\
-X_{\epsilon} & =\delta_{\phi_{\epsilon}}\left(\tilde{\alpha}_{\epsilon}\right)-\phi_{*} Y_{\epsilon}-\phi^{*} Z_{\epsilon} ; \\
\xi_{\mathcal{G}_{\epsilon}} & =\delta_{\mathcal{G}_{\epsilon}} Z_{\epsilon} .
\end{aligned}
$$

By the first and third equation, if $\varphi_{\epsilon}$ and $\psi_{\epsilon}$ are the time-dependent flows starting at zero of the vector fields $\left\{-Y_{\epsilon}\right\}_{\epsilon}$ and $\left\{Z_{\epsilon}\right\}_{\epsilon}$ respectively, then they define the equivalences with the corresponding constant deformations of Lie groupoids. We claim that these equivalences can 
be used to prove the triviality of $\left\{\mathcal{H}_{\epsilon} \stackrel{\phi_{\epsilon}}{\longrightarrow} \mathcal{G}_{\epsilon}\right\}_{\epsilon}$. In fact, by using Theorem 2.5.2, we will show that the family of morphisms $\left\{f_{\epsilon}:=\psi_{\epsilon}^{-1} \circ \phi_{\epsilon} \circ \varphi_{\epsilon}\right\}_{\epsilon}$ is a trivial deformation in the sense of the definition at the beginning of Section 2.2. Note that such a family satisfies,

$$
\begin{aligned}
\frac{d}{d \epsilon} f_{\epsilon}(h) & =\left(\frac{d}{d \epsilon} \psi_{\epsilon}^{-1}\right)\left(\phi_{\epsilon} \circ \varphi_{\epsilon}(h)\right)+d \psi_{\epsilon}^{-1}\left(X_{\epsilon}\left(\varphi_{\epsilon}(h)\right)\right)-d\left(\psi_{\epsilon}^{-1} \circ \phi_{\epsilon}\right)\left(Y_{\epsilon}\left(\varphi_{\epsilon}(h)\right)\right) \\
& =-d \psi_{\epsilon}^{-1}\left(Z_{\epsilon}\left(\phi_{\epsilon} \circ \varphi_{\epsilon}(h)\right)\right)+d \psi_{\epsilon}^{-1}\left(X_{\epsilon}\left(\varphi_{\epsilon}(h)\right)\right)-d \psi_{\epsilon}^{-1}\left[d \phi_{\epsilon}\left(Y_{\epsilon}\left(\varphi_{\epsilon}(h)\right)\right)\right] \\
& =d \psi_{\epsilon}^{-1}\left[X_{\epsilon}\left(\varphi_{\epsilon}(h)\right)-\left(\left(\phi_{\epsilon}\right)_{*} Y_{\epsilon}\right)_{\left(\varphi_{\epsilon}(h)\right)}-\left(\phi_{\epsilon}^{*} Z_{\epsilon}\right)_{\left(\varphi_{\epsilon}(h)\right)}\right] \\
& =-d \psi_{\epsilon}^{-1}\left(\delta_{\phi_{\epsilon}}\left(\tilde{\alpha}_{\epsilon}\right)\left(\varphi_{\epsilon}(h)\right)\right) \quad(\text { by transgression equations above }) \\
& =-d \psi_{\epsilon}^{-1}\left(\delta_{\phi_{\epsilon} \circ \varphi_{\epsilon}}\left(\bar{\alpha}_{\epsilon}\right)(h)\right) \quad\left(\bar{\alpha}_{\epsilon}=\varphi_{\epsilon}^{*} \tilde{\alpha}_{\epsilon}\right) \\
& =-\delta_{\psi_{\epsilon}^{-1} \circ \phi_{\epsilon} \circ \varphi_{\epsilon}}\left(\alpha_{\epsilon}\right)(h) \quad\left(\alpha_{\epsilon}=-\left(\psi_{\epsilon}^{-1}\right)_{*} \bar{\alpha}_{\epsilon}\right) \\
& =\delta_{f_{\epsilon}}\left(\alpha_{\epsilon}\right)(h),
\end{aligned}
$$

where the second equality follows from the fact that $\left(\frac{d}{d \epsilon} \psi_{\epsilon}^{-1}\right)\left(\psi_{\epsilon}(h)\right)+d \psi_{\epsilon}^{-1}\left(Z_{\epsilon}\left(\psi_{\epsilon}(h)\right)\right)=0$ (apply $\frac{d}{d \epsilon}$ to $\psi_{\epsilon}^{-1} \circ \psi_{\epsilon}=I d$ ). Therefore, by Theorem 2.5.2, $\psi_{\epsilon}^{-1} \circ \phi_{\epsilon} \circ \varphi_{\epsilon}=I_{\sigma_{\epsilon}} \circ \phi$, for $\epsilon$ small enough, as claimed.

\subsection{Particular cases and relation between (sub)complexes}

In view that the complex $C_{\text {def }}^{*}(\mathcal{H}, \phi, \mathcal{G})$ concerns the most general type of deformations of the three structures $(\mathcal{H}, \phi, \mathcal{G})$, in this section we consider particular cases of deformations of the triple and we look at their relation with some subcomplexes of $C_{d e f}^{*}(\mathcal{H}, \phi, \mathcal{G})$. We begin by the simplest case.

\subsection{1 $\mathcal{H}$ and $\mathcal{G}$ fixed}

In this case, we get a deformation of a Lie groupoid morphism. Of course, a deformation $\left\{\phi_{\epsilon}\right\}_{\epsilon}$ of $\phi$ can be viewed as a deformation $\left\{\mathcal{H} \stackrel{\phi_{\epsilon}}{\longrightarrow} \mathcal{G}\right\}_{\epsilon}$ of the triple $(\mathcal{H}, \phi, \mathcal{G})$. This fact is expressed, in cohomological terms, by the injection $C_{d e f}^{*}(\phi) \longrightarrow C_{d e f}^{*}(\mathcal{H}, \phi, \mathcal{G})$

$$
X \mapsto(0,-X, 0)
$$

Moreover, this map takes the infinitesimal cocycle of $\left\{\phi_{\epsilon}\right\}_{\epsilon}$ to the infinitesimal cocycle of $\left\{\mathcal{H} \stackrel{\phi_{\epsilon}}{\longrightarrow} \mathcal{G}\right\}_{\epsilon}$. Therefore, in this case, the relevant subcomplex controlling deformations of this type is given by $\{0\} \oplus C_{d e f}^{*}(\phi) \oplus\{0\}$. 


\subsection{2 $\mathcal{G}$ fixed}

In this case, the relevant subcomplex is $C_{\text {def }}^{*+1}(\mathcal{H}) \oplus C_{\text {def }}^{*}(\phi) \oplus\{0\}$. In fact, it is not hard to see that a deformation like $\left\{\left(\mathcal{H}_{\epsilon}, \phi_{\epsilon}, \mathcal{G}\right)\right\}_{\epsilon}$ is governed by the mapping-cone complex $M C^{*}\left(\left(\phi_{0}\right)_{*}\right)$ (see $\left.(2.9)\right)$ (associate the cocycle $\left.\left(\xi_{\mathcal{H}},-X\right) \in C_{\text {def }}^{2}(\mathcal{H}) \oplus C_{\text {def }}^{1}(\phi)\right)$. Thus the (injective) chain map $M C^{*}\left(\phi_{*}\right) \longrightarrow C_{\text {def }}^{*}(\mathcal{H}, \phi, \mathcal{G})$

$$
(c, X) \mapsto(-1)^{\operatorname{deg}(c)}(c, X, 0)
$$

shows that the subcomplex $C_{d e f}^{*+1}(\mathcal{H}) \oplus C_{d e f}^{*}(\phi) \oplus\{0\}$ controls the deformations of the triple when we fix the groupoid $\mathcal{G}$.

Important examples of this type of deformations are given by deformations of Lie subgroupoids $\left\{H_{\epsilon} \subset \mathcal{G}\right\}_{\phi_{\epsilon}}$. Therefore, the injection (2.10) above reflects, in cohomological terms, the fact that a deformation of subgroupoids $\left.\left\{\mathcal{H}_{\epsilon}\right\}_{\{} \phi_{\epsilon}\right\}$ induces the deformation $\left\{\left(\mathcal{H}_{\epsilon} \stackrel{\phi_{e}}{\longrightarrow} \mathcal{G}\right)\right\}_{\epsilon}$ of the triple $(\mathcal{H}, \iota, \mathcal{G})$. In terms of the complex $M C^{*}\left(\phi_{*}\right)$ above, we obtain the following proposition whose proof is similar to that of Theorem 2.6.2.

Proposition 2.7.1 Let $\left\{\tilde{\mathcal{H}}_{\epsilon}\right\}_{\epsilon} \stackrel{\left\{\phi_{\epsilon}\right\}}{\longrightarrow} \mathcal{G}$ be an s-constant deformation of $\mathcal{H} \subset \mathcal{G}$, with $\mathcal{H}$ compact. If the family of associated cocycles $\left\{\left(\xi_{\mathcal{H}_{\epsilon}},-X_{\epsilon}\right) \in C_{d e f}^{2}\left(\mathcal{H}_{\epsilon}\right) \oplus C_{d e f}^{1}\left(\phi_{e}\right)\right\}_{\epsilon}$ is transgressed by an smooth family of cochains $\left\{\left(T_{\epsilon}, \alpha_{\epsilon}\right)\right\}_{\epsilon}$, the deformation $\left\{\mathcal{H}_{\epsilon} \subset \mathcal{G}\right\}_{\epsilon}$ is trivial.

Note the analogy of the statement above with that of Theorem 2.5.6. In fact, by using the fact that every $\left(\phi_{\epsilon}\right)_{*}$ is injective, it is not hard to see that the condition of $\left\{\left[\bar{X}_{\epsilon}\right] \in C_{d e f}^{1}\left(\mathcal{H}_{\epsilon} \subset\right.\right.$ $\mathcal{G})\}_{\epsilon}$ being transgressed by an smooth family is equivalent to the one of $\left\{\left(\xi_{\mathcal{H}_{\epsilon}},-\bar{X}_{\epsilon}\right)\right\}_{\epsilon}$ being transgressed by an smooth family of cochains (this is not true in general for any deformation $\left\{\mathcal{H}_{\epsilon} \stackrel{\phi_{e}}{\mathcal{G}}\right\}_{\epsilon}$ of the triple $(\mathcal{H}, \phi, \mathcal{G})$ : the smoothness of the family of primitives of $\left(\xi_{\mathcal{H}_{\epsilon}},-\bar{X}_{\epsilon}\right)$ would not be guaranteed just by assuming the smoothness of the family of primitives of $\left.\left[\bar{X}_{\epsilon}\right]\right)$. In this sense, Theorem 2.5.6 and Proposition 2.7.1 above prove the same thing, with the same assumptions, in terms of two different complexes. We prove now that these two complexes have the same cohomology.

Proposition 2.7.2 Let $\iota: \mathcal{H} \hookrightarrow \mathcal{G}$ be the inclusion map of the subgroupoid $\mathcal{H}$. The short exact sequence (2.2) determined by the deformation complex of subgroupoids induces an isomorphism, $\mathcal{F}: H_{\text {def }}^{*}(\mathcal{H} \subset \mathcal{G}) \longrightarrow H^{*}\left(M C\left(\iota_{*}\right)\right)$, between the deformation cohomology of $\mathcal{H} \subset \mathcal{G}$ as subgroupoid and the cohomology of the mapping-cone complex of the cochain-map $\iota_{*}: C_{d e f}^{*}(\mathcal{H}) \longrightarrow C_{d e f}^{*}(i)$

Proof. In order to define $\mathcal{F}$, we look at its action on cocycles. Let $[c] \in C_{d e f}^{k}(\mathcal{H} \subset \mathcal{G})$ be a cocycle. By the exactness of

$$
0 \rightarrow C_{\text {def }}^{*}(\mathcal{H}) \stackrel{\iota_{*}}{\longrightarrow} C_{\text {def }}^{*}(\iota) \longrightarrow C_{\text {def }}^{*}(\mathcal{H} \subset \mathcal{G}) \rightarrow 0
$$


in $C_{\text {def }}^{*}(\mathcal{H} \subset \mathcal{G})$, there exists an element in $C_{\text {def }}^{k}(\iota)$ which projects to $[c]$; assume it is $c$. Thus $\delta_{\phi}(c) \in C_{\text {def }}^{k+1}(\iota)$ projects to zero in $C_{\text {def }}^{k+1}(\mathcal{H} \subset \mathcal{G})$. Therefore, $\delta_{\phi}(c)=\iota_{*}(\xi)$, for an unique element $\xi \in C_{d e f}^{k+1}(\mathcal{H})$. In this way, we define $\mathcal{F}$ by,

$$
\mathcal{F}(\overline{[c]}):=[(\xi,-c)] \in H^{k}\left(M C^{*}\left(\iota_{*}\right)\right)
$$

The fact that $(\xi,-c) \in C_{d e f}^{k+1}(\mathcal{H}) \oplus C_{d e f}^{k}(\iota)=M C^{k}\left(\iota_{*}\right)$ is a cocycle follows from the injectivity of $\iota_{*}$. To check that $\mathcal{F}$ is well-defined and injective is a straightforward computation. An inverse for $\mathcal{F}$ is given by $\mathcal{I}([(\xi, c)]):=\overline{[-c]}$, which is well-defined and injective (a straightforward computation again).

Remark 2.7.3 Following the arguments of the previous proof, one has in fact a general property for any injective chain map $\Phi: \mathcal{A} \longrightarrow \mathcal{B}$ between complexes: there is an isomorphism between the cohomology groups of the cokernel complex of $\Phi$ and the ones of the mapping-cone complex of $\Phi$.

Thus, in view of Proposition 2.7.1 and Proposition 2.7.2, we can regard the complex $M C^{*}\left(\iota_{*}\right)$ as another complex controlling deformations of subgroupoids.

\subsection{General deformations}

In this section we show how the correspondence 'deformations-cocycles', explained in Section 2.4, for strict deformations of Lie subgroupoids also apply for general deformations of Lie subgroupoids (remark 2.2.5). That is, we associate a cohomology class to a general deformation of subgroupoids.

In view that this process is similar to that described in Crainic et al. (2015a) for general deformations of Lie groupoids, we indicate here the main steps for the case of Lie subgroupoids.

Let

$$
(\tilde{H} \underset{\tilde{t}}{\stackrel{\tilde{s}}{\longrightarrow}} \tilde{N} \stackrel{\pi}{\longrightarrow} I) \stackrel{\phi}{\longrightarrow}\left(\mathcal{G} \times I \frac{s \times i d_{I}}{t \times i d_{I}} M \times I \stackrel{p r_{I}}{\longrightarrow} I\right)
$$

be a general deformation of $\mathcal{H} \subset \mathcal{G}$ as subgroupoid. Take $\tilde{X} \in \mathfrak{X}(\tilde{H})$ a tranverse vector field for $\tilde{H}$ (i.e., $\tilde{X}$ projects by $\tilde{s}$ to a vector field on $\tilde{N}$ which in turn projects to the vector field $\partial / \partial \epsilon \in \mathfrak{X}(I))$. Then, if we denote by $p r_{\mathcal{G}}: \mathcal{G} \times I \longrightarrow \mathcal{G}$ the projection to $\mathcal{G}$ and by $\tilde{H}_{0}$ the fiber of $\tilde{H}$ over 0 , the element $\bar{X} \in C_{d e f}^{*}(i)$ defined by

$$
\bar{X}:=\left.\left(\left(p r_{\mathcal{G}}\right)_{*}(\phi)_{*} \tilde{X}\right)\right|_{\tilde{H}_{0}}
$$

induces the cocycle $X:=[\bar{X}] \in C_{d e f}^{1}(\mathcal{H} \subset \mathcal{G})$. In fact, by (Crainic et al. (2015a), Prop. 5.12), $\delta_{\tilde{H}}(\tilde{X}) \in C_{\text {def }}^{2}(\tilde{H})$ restricted to $\tilde{H}_{0}$ is tangent to $\tilde{H}_{0} \subset \tilde{H}$, therefore $\delta_{\phi}\left(\phi_{*} \tilde{X}\right)=\phi_{*}\left(\delta_{\tilde{H}}(\tilde{X})\right) \in$ $C_{d e f}^{2}(\phi)$ when restricted to $\tilde{H}_{0} \subset \tilde{H}$ is tangent to $\iota\left(\tilde{H}_{0}\right) \subset \mathcal{G}$. Thus, due to the fact that 
$\left.\delta_{\phi}\left(\phi_{*} \tilde{X}\right)\right|_{\tilde{H}_{0}}=\left(\delta_{\iota}\left(\left.\left(\left(p r_{\mathcal{G}}\right)_{*} \phi_{*} \tilde{X}\right)\right|_{\tilde{H}_{0}}\right), 0\right)$, we get that $\delta_{\iota}(\bar{X}) \in C_{d e f}^{2}(\iota)$ is tangent to $\iota\left(\tilde{H}_{0} \subset\right.$ $\mathcal{G})$; that is, $X=[\bar{X}]$ is a cocycle in $C_{d e f}^{1}(\mathcal{H} \subset \mathcal{G})$. The corresponding cohomology class of $X$ is called the associated cohomology class of the deformation $(\tilde{H}, \phi)$. That this class does not depend on the choice of the tranverse vector field is proved in a similar way to that of (iii) in Proposition 5.12 of Crainic et al. (2015a). 


\section{Chapter 3}

\section{Application: Rigidity of Lie group morphisms and Lie subgroups}

This chapter aims to use the deformation theory developed in the previous work in the context of the Lie groups theory. This will give us, under compactness conditions, rigidity results for Lie group morphisms and Lie subgroups in relation with those made in Nijenhuis and Richardson Jr (1967) and Coppersmith (1977). For that we will express several of the previous results in the language of Lie groups, which provides a more direct analogy with the arguments of the classical Moser's trick in symplectic geometry. In particular, this will indicate that for the case of Lie group morphisms and Lie subgroups, the space of cochains of the Lie group complex is the analogue to the space of forms on $M$ (the space cochains of the de Rham complex), and the deformation cocycles are the analogue to the cocycles $\frac{d}{d \epsilon} \omega_{\epsilon} \in \Omega_{d R}^{2}(M)$ appearing in the classical Moser's theorem. The last section of this chapter explains the rigidity results and compares them with some related works existing in the literature.

\subsection{Lie group cohomology}

In this section we briefly recall the Lie group complex associated to a representation $V$ of a Lie group $G$, and describe the representations useful for our purposes. Having in mind the classical Moser's theorem in symplectic geometry, in our approach, the corresponding Lie group cohomology can be seen as the analogue to the de Rham cohomology, they will play the same role as the de Rham cohomology groups for deformations of symplectic forms on a manifold, as explained in McDuff and Salamon (1998). As in the previous sections, we study deformation problems in an infinitesimal way. In this sense, to each deformation, we associate certain cocycles which preserves the infinitesimal information, and look at its corresponding cohomology classes. With this in mind, we now describe the relevant cohomology theory for us: the Lie group cohomology. 
Let $G$ be a Lie group and $\rho: G \rightarrow G L(V)$ be a representation of $G$. The cochain complex of $G$ with coefficients in $V$ is defined as follows: the cochains $C^{k}(G, V)$ of degree $k$ are given by the smooth functions of $G^{k}$ with values in $V, C^{\infty}\left(G^{k}, V\right)$, with differential $\delta_{\rho}: C^{k}(G, V) \rightarrow C^{k+1}(G, V)$ described by

$$
\begin{aligned}
\delta_{\rho} c\left(g_{1}, \ldots g_{k+1}\right):=\rho\left(g_{1}\right) c\left(g_{2}, \ldots, g_{k+1}\right) & +\sum_{i=1}^{k}(-1)^{i} c\left(g_{1}, \ldots g_{i} g_{i+1}, \ldots g_{k+1}\right) \\
& +(-1)^{k+1} c\left(g_{1}, \ldots, g_{k}\right) .
\end{aligned}
$$

For any representation $\rho$ of $G$, we have $\delta_{\rho}^{2}=0$; the resulting cohomology is denoted by $H_{\rho}^{k}(G, V)$.

For this chapter, the following examples will be useful for us.

Example 3.1.1 Take $V=\mathfrak{g}$, the Lie algebra associated to $G$, along with the adjoint representation of $G, A d: G \longrightarrow G L(\mathfrak{g})$. We denote the differential by $\delta$, and the cohomology by $H^{*}(G, \mathfrak{g})$.

Example 3.1.2 Take $V=\mathfrak{g}$. If $\phi: H \rightarrow G$ is a morphism, $\rho=A d \circ \phi: H \rightarrow G L(\mathfrak{g})$ is a representation of $H$. In this case, we denote the differential by $\delta_{\phi}$ and the cohomology by $H_{\phi}^{*}(H, \mathfrak{g})$.

Example 3.1.3 Let $H \subset G$ be a Lie subgroup of $G$, take $V=\mathfrak{g} / \mathfrak{h}$, where $\mathfrak{h} \subset \mathfrak{g}$ is the Lie subalgebra associated to $H$. We have a representation of $H$ on $\mathfrak{g} / \mathfrak{h}$ induced by the representation of $H$ on $\mathfrak{g}$ of previous example (with $\phi=\iota: H \hookrightarrow G$ ). In this case, we denote the differential by $\delta$ (again) and the cohomology by $H^{*}(H, \mathfrak{g} / \mathfrak{h})$.

Remark 3.1.4 Note that, by putting together the previous two examples, if $\phi: H \rightarrow G$ is a morphism and $\mathfrak{h}$ is the Lie algebra of $H$, one also obtains a complex with differential and cohomology that we denote by $\bar{\delta}_{\phi}$ and $H_{\phi}^{k}(H, \mathfrak{g} / \tilde{\mathfrak{h}})$ respectively, where $\tilde{\mathfrak{h}}$ is the Lie subalgebra of $\phi(H)$.

Remark 3.1.5 Observe that, with the same notation of the previous remark, there is a natural cochain-map between these complexes:

$$
\phi_{*}: C^{*}(H, \mathfrak{h}) \rightarrow C_{\phi}^{*}(H, \tilde{\mathfrak{h}}) \subset C_{\phi}^{*}(H, \mathfrak{g}) .
$$

\subsection{Deformations of morphisms and Lie subgroups}

For clarity, in this section we adapt the definition of deformation of Lie groupoids, morphisms and Lie subgroupoids to the context of Lie groups. We start by considering the key facts of deformation of Lie groups.

Let $G$ be a Lie group with multiplication $m: G \times G \rightarrow G$. 
Definition 3.2.1 A deformation of the Lie group $G,\left\{\left(G, m_{\epsilon}\right)\right\}_{\epsilon \in I}$, is a smooth family of maps $\left\{m_{\epsilon}: G \times G \rightarrow G\right\}$ with $m_{0}=m$ (i.e. a deformation of the map $m$ ), such that every $\left(G, m_{\epsilon}\right)$ is a Lie group. We denote the Lie group $\left(G, m_{\epsilon}\right)$ by $\tilde{G}_{\epsilon}$. The deformation such that $m_{\epsilon}=m_{0}$ for all $\epsilon$ is called the constant deformation of $G$.

Example 3.2.2 If $\left\{F_{\epsilon}: G \rightarrow G\right\}$ is a smooth family of bijective functions with $F_{0}$ an automorphism of $G$, one obtains a deformation $\left\{m_{\epsilon}\right\}$ by making $m_{\epsilon}(g, h):=F_{\epsilon}^{-1}\left(m\left(F_{\epsilon}(g), F_{\epsilon}(h)\right)\right)$. In other words, this deformation is determined by the flow of a time-dependent vector field (the one induced by the family $\left.\left\{F_{\epsilon}\right\}_{\epsilon}\right)$. This type of deformation is called a trivial deformation of the Lie group (see definition below).

Definition 3.2.3 (Equivalence of deformations) Two deformations of $G,\left\{\tilde{G}_{\epsilon}\right\}$ and $\left\{\tilde{G}_{\epsilon}^{\prime}\right\}$ are called equivalent if there exists a smooth family, $\left\{F_{\epsilon}\right\}$, of isomorphisms $F_{\epsilon}: \tilde{G}_{\epsilon} \rightarrow \tilde{G}_{\epsilon}^{\prime}$ such that $F_{0}=I d_{G}$. The deformation $\left\{\tilde{G}_{\epsilon}\right\}$ is said to be trivial if it is equivalent to the constant deformation. The Lie group $G$ is called rigid if any deformation of $G$ is trivial.

Remark 3.2.4 If $\left\{\tilde{G}_{\epsilon}\right\}$ is a deformation of $G$, one can always assume that the identity of $G$ remains fixed by the deformation. (We can always build an equivalent deformation which keeps the identity fixed).

There is a natural cochain map $\Phi$ which relates the deformation cohomology of $G$ to the group cohomology of the adjoint representation of $G$. Namely $\Phi: C_{d e f}^{k}(G) \longrightarrow C_{A d}^{k}(G, \mathfrak{g})$,

$$
\Phi(c)\left(g_{1}, \ldots, g_{k}\right)=r_{g_{1}^{-1}}\left(c\left(g_{1}, \ldots, g_{k}\right)\right)
$$

Note that $\Phi$ is an isomorphism.

Now we pass to give an infinitesimal description of deformations of Lie groups, by following the general principle of deformation theory: associate to every deformation a deformation cocycle in an appropriate cohomology. In this case, such a cocycle is a rewriting of that of deformation of Lie groupoids determined by the isomorphism $\Phi$. Namely, given $\left\{\tilde{G}_{\epsilon}\right\}$ a deformation of the Lie group $G$, its deformation cocycle is:

$$
\xi_{0}(g, h):=-r_{m_{0}(g, h)}^{-1}\left(\left.\frac{d}{d \epsilon}\right|_{\epsilon=0} m_{\epsilon}(g, h)\right) \in T_{e} G \equiv \mathfrak{g} .
$$

By its definition, $\xi_{0}$ has the infinitesimal information of the deformation close to $G_{0}$, and it is one of the main ingredients to study the rigidity question for Lie groups, as shown below. To see that $\xi_{0}$ is in fact a 2-cocycle it suffices to take derivative at $\epsilon=0$ of the associativity property of the multiplication $m_{\epsilon}$. Similarly, one can consider the 2-cocycles, $\xi_{\epsilon}$, in time different from $\epsilon=0$.

The rigidity question for Lie groups is based on the following proposition whose proof we sketch in order to motivate the proofs of the rigidity results of morphisms and Lie subgroups below. 
Proposition 3.2.5 Crainic et al. (2015a) Let $\left\{\tilde{G}_{\epsilon}\right\}$ be a deformation of the compact Lie group $\left(G, m_{0}\right)$. Consider the (smooth) family of deformation cocycles $\left\{\xi_{\epsilon} \in C^{2}\left(\tilde{G}_{\epsilon}, \mathfrak{g}\right)\right\}$. If there exists a smooth family, $\left\{X_{\epsilon} \in C^{1}\left(\tilde{G}_{\epsilon}, \mathfrak{g}\right)\right\}_{\epsilon}$, of primitives of $\left\{\xi_{\epsilon}\right\}$, i.e., elements such that $\delta_{\epsilon} X_{\epsilon}=\xi_{\epsilon}$, the deformation is trivial.

Proof. Given the family $\left\{X_{\epsilon}: G \cong \tilde{G}_{\epsilon} \rightarrow \mathfrak{g}\right\}$, we can construct a family of vector fields on $G,\left\{\bar{X}_{\epsilon}:=r_{g}^{\epsilon}\left(X_{\epsilon}(g)\right)\right\}$, where $r^{\epsilon}$ is the right translations on $\tilde{G}_{\epsilon}$. The family of isomorphisms $F_{\epsilon}: G \longrightarrow G_{\epsilon}$ arisen as the flow of the time-dependent vector field $\left\{\bar{X}_{\epsilon}\right\}_{\epsilon \in I}$ gives rise to the equivalence of the deformations.

Remark 3.2.6 Note that, in the previous proof, the cohomological condition $\delta_{\epsilon} X_{\epsilon}=\xi_{\epsilon}$ is which guarantees that the induced flow is by isomorphisms of Lie groups.

Therefore, by using the homotopy operator of the Lie group complex (which exists if the Lie group is compact) is obtained the following

Corollary 3.2.7 (?, Thm. 7.1)

Every compact Lie group is rigid.

We now define deformations of Lie group morphisms and Lie subgroups. Given $H, G$ Lie groups and $\phi: H \rightarrow G$ a Lie group morphism, we call the family of maps $\left\{\phi_{\epsilon}\right\}_{\epsilon \in I}$ a deformation of $\phi$ if this is a smooth family of morphisms $\phi_{\epsilon}: H \rightarrow G$ with $\phi_{0}=\phi$. Clearly, if $\left\{\epsilon \mapsto g_{\epsilon}\right\}$ is a smooth curve in $G$ with $g_{0}=e \in G$ then, by conjugating $\phi$ by every $g_{\epsilon}$, one obtains a deformation of $\phi, \phi_{\epsilon}:=I_{g_{\epsilon}} \circ \phi$. This is called a trivial deformation of $\phi$. Also, we will say that two deformations of $\phi,\left\{\phi_{\epsilon}\right\}$ and $\left\{\psi_{\epsilon}\right\}$, are equivalent if there exist a smooth curve $\left\{\epsilon \mapsto g_{\epsilon}\right\}$ in $G$, with $g_{0}=e$, such that $\psi_{\epsilon}=I_{g_{\epsilon}} \circ \phi_{\epsilon}$, for all $\epsilon$. The morphism $\phi$ is called rigid if any deformation of $\phi$ is trivial.

The definition for Lie subgroups is as follows. Let $H \subset(G, m)$ be a Lie subgroup, then $\left(H,\left.m\right|_{H}\right)$ is a Lie group, where $\left.m\right|_{H}: H \times H \rightarrow H$.

Definition 3.2.8 A deformation of $H \subset G$ as Lie subgroup is a deformation of $\left.m\right|_{H}$ as Lie group, $\left\{\tilde{H}_{\epsilon}\right\}$, together with a smooth family of injective morphisms $\left\{\phi_{\epsilon}: \tilde{H}_{\epsilon} \rightarrow G\right\}$ with $\phi_{0}=\iota: H \hookrightarrow G$. We will denote this deformation by $\left\{H_{\epsilon}\right\}_{\phi}$ or by $\left\{H_{\epsilon}\right\}$ when there is no confusion. The deformation such that $\tilde{H}_{\epsilon}=H$ and $\phi_{\epsilon}=\iota$ will be called the constant deformation of $H$, i.e., $H_{\epsilon}=H$ for all $\epsilon$.

Definition 3.2.9 (Equivalence of deformations of Lie subgroups) Given two deformations $\left\{H_{\epsilon}\right\}$ and $\left\{H_{\epsilon}^{\prime}\right\}$ of $H \subset G$ as Lie subgroup. We will say they are equivalent if there exist a smooth curve $g_{\epsilon}$ in $G$, with $g_{0}=e$, such that $H_{\epsilon}^{\prime}=I_{g_{\epsilon}}\left(H_{\epsilon}\right)$ for every $\epsilon \in I$. The deformation is said to be trivial if it is equivalent to the constant deformation. The subgroup $H$ is called rigid if every deformation of $H$ is trivial. 
Remark 3.2.10 Note that, by the injectivity of $\phi_{\epsilon}$, if two deformations $\left\{H_{\epsilon}\right\}_{\phi_{\epsilon}}$ and $\left\{H_{\epsilon}^{\prime}\right\}_{\psi_{\epsilon}}$ are equivalent there exist a unique family of isomorphisms $\left\{F_{\epsilon}: H_{\epsilon} \rightarrow H_{\epsilon}^{\prime}\right\}_{\epsilon}$ such that

$$
\psi_{\epsilon} \circ F_{\epsilon}=I_{g(\epsilon)} \circ \phi_{\epsilon}
$$

and since every $\psi_{\epsilon}$ is an immersion, this family is smooth. (Recall that injective morphism of Lie groups is also an immersion).

We now apply the 'general principle' of deformation theory to deformations of morphisms and subgroups.

\subsubsection{Relation with deformation complexes and associated cocycles}

Analogous to the deformation complex of a Lie group $G$, if $\phi_{0}: H \longrightarrow G$ is a morphism of Lie groups, there exists a natural cochain-map $\Psi: C_{d e f}^{*}\left(\phi_{0}\right) \stackrel{\cong}{\longrightarrow} C_{\phi_{0}}^{*}(H, \mathfrak{g})$ given by $\Psi(c)\left(h_{1}, \ldots, h_{k}\right)=r_{\phi_{0}\left(h_{1}\right)}^{-1}\left(c\left(h_{1}, \ldots, h_{k}\right)\right)$. Then if $\left\{\phi_{\epsilon}\right\}_{\epsilon \in I}$ is a deformation of $\phi_{0}$, we have the following 1-cocycle in $C_{\phi_{0}}^{*}(H, \mathfrak{g})$ associated to the deformation

$$
\tilde{X}_{0}(h):=\left.r_{\phi_{0}(h)}^{-1} \frac{d}{d \epsilon}\right|_{\epsilon=0} \phi_{\epsilon}(h)
$$

which is the image by $\Psi$ of the deformation 1-cocycle associated to $\left\{\phi_{\epsilon}\right\}_{\epsilon}$. The element $\tilde{X}_{0}$ will be also called the infinitesimal cocycle associated to the deformation $\left\{\phi_{\epsilon}\right\}$. Hence, Proposition 2.4.1 is translated as follows.

Proposition 3.2.11 Let $\left\{\phi_{e}\right\}_{\epsilon}$ be a deformation of $\phi_{0}$. The corresponding cohomology class in $H_{\phi_{0}}^{1}(H, \mathfrak{g})$ of the cocycle $\tilde{X}_{0}$ defined above, depends only on the equivalence class of the deformation.

For Lie subgroups the situation is similar: since the cochain-map $\Psi$ defined above (making $\left.\phi_{0}=\iota: H \hookrightarrow G\right)$ restricts to $\left.\Psi\right|_{C_{d e f}^{*}(H)}: C_{d e f}^{*}(H) \longrightarrow C^{*}(H, \mathfrak{h})$, the map $\Psi$ induces the isomorphism of complexes $\bar{\Psi}: C_{d e f}^{*}(H \subset G) \longrightarrow \frac{C_{\iota}^{*}(H, \mathfrak{g})}{C^{*}(H, \mathfrak{h})}$; and this latter complex is clearly related to $C^{*}(H, \mathfrak{g} / \mathfrak{h})$ by the isomorphism $\mathcal{F}: \frac{C_{l}^{*}(H, \mathfrak{g})}{C^{*}(H, \mathfrak{h})} \longrightarrow C^{*}(H, \mathfrak{g} / \mathfrak{h})$,

$$
\mathcal{F}([c])\left(h_{1}, \ldots, h_{k}\right)=\left[c\left(h_{1}, \ldots, h_{k}\right)\right]_{\mathfrak{g} / \mathfrak{h}} .
$$

Therefore if $\left\{H_{\epsilon}\right\}_{\phi}$ is a deformation of $H \subset G$, the deformation cocycle of $\left\{H_{\epsilon}\right\}_{\phi}$ has image $($ by $\mathcal{F} \circ \bar{\Psi}) X_{0} \in C^{1}(H, \mathfrak{g} / \mathfrak{h})$ given by $X_{0}(h)=\left[\tilde{X}_{0}(h)\right]_{\mathfrak{g} / \mathfrak{h}}$, where

$$
\tilde{X}_{0}(h):=\left.r_{\iota(h)}^{-1} \frac{d}{d \epsilon}\right|_{\epsilon=0} \phi_{\epsilon}(h) \quad \in \mathfrak{g}, \quad\left(\tilde{X}_{0} \in C_{\iota}^{1}(H, \mathfrak{g})\right)
$$

The cocycle $X_{0}$ will be also called the infinitesimal cocycle associated to the deformation $\left\{H_{\epsilon}\right\}_{\phi}$ of $H \subset G$. Thus, proposition 2.4.4 turns, 
Proposition 3.2.12 Let $\left\{H_{\epsilon}\right\}_{\phi}$ be a deformation of $H \subset G$. The corresponding cohomology class in $H_{\iota}^{1}(H, \mathfrak{g} / \mathfrak{h})$ of the cocycle $X_{0}$, above described, depends only on the equivalence class of the deformation.

\subsubsection{Rigidity}

In order to prove the rigidity results for Lie group morphisms as an application of the deformation theory of morphisms of groupoids, we write the Theorem 2.5.2 in the language of Lie groups theory.

Proposition 3.2.13 Let $\left\{\phi_{\epsilon}\right\}$ be a deformation of $\phi_{0}: H \rightarrow G$. If every associated 1-cocycle $\tilde{X}_{\epsilon}$ is exact and equal to $\delta_{\phi_{\epsilon}}\left(u^{\epsilon}\right)$, with $\left\{u^{\epsilon}\right\}_{\epsilon}$ a smooth family of elements in $\mathfrak{g}$, then the deformation $\left\{\phi_{\epsilon}\right\}_{\epsilon}$ is trivial.

Theorem 3.2.14 (Rigidity of morphisms)

Let $\phi: H \longrightarrow G$ be a morphism of Lie groups. If $H$ is compact, $\phi$ is rigid.

Proof. Let $\left\{\phi_{\epsilon}\right\}$ any deformation of $\phi$. If $H$ is compact, each $H_{\phi_{\epsilon}}^{1}(H, \mathfrak{g})$ vanishes. One primitive of $\tilde{X}_{\epsilon}$ is given by $u^{\epsilon}=-\int_{H} \tilde{X}_{\epsilon}(h) d h \in \mathfrak{g}$, where the integral is taken w.r.t. the Haar's measure of $H$. Thus, since $\tilde{X}_{\epsilon}$ is a smooth family $\left(\tilde{X}_{\epsilon}: H \rightarrow \mathfrak{g}\right)$, the family of elements $\left\{u^{\epsilon}\right\}$ of $\mathfrak{g}$ is smooth. Now, apply the previous proposition.

Remark 3.2.15 In Lee (1974) was already studied a little different notion of rigidity of morphisms of Lie groups. There, the author considers the action of $G$ on $\operatorname{Hom}(H, G)$ (the set of continuous homomorphisms) given by $g \cdot \phi:=I_{g} \circ \phi$ and says that $\phi$ is rigid if the orbit $O_{\phi}$ of $\phi$ is an open subset of $\operatorname{Hom}(H, G)$ with the compac-open topology. Thus, such a notion of rigidity of $\phi$ implies that any deformation of $\phi$ is of the form $I_{g_{\epsilon}} \circ \phi$, for $g_{\epsilon}$ a curve in $G$. However this curve $g_{\epsilon}$ is not necessarily smooth. Therefore, this notion of rigidity does not necessarily imply the ours. Conversely, if $\phi$ is rigid in the sense of our notion, still that any small curve in $\operatorname{Hom}(H, G)$ starting at $\phi$ is inside the orbit of $\phi$, we can not guarantee that $\phi$ is rigid in the sense of Lee (1974).

Theorem 3.2.16 (Rigidity of Lie subgroups)

Every compact Lie subgroup is rigid.

Proof. Let $H \subset G$ be a compact Lie subgroup of $G$ and $\left\{H_{\epsilon}\right\}_{\phi}$ be a deformation of $H \subset G$. Consider the family of 1-cochains associated to the deformation,

$$
\tilde{X}_{\epsilon}(h)=r_{\phi_{\epsilon}(h)}^{-1}\left(\left.\frac{d}{d \lambda}\right|_{\lambda=\epsilon} \phi_{\lambda}(h)\right) \in \mathfrak{g}, \quad\left(\tilde{X}_{\epsilon} \in C_{\phi_{\epsilon}}^{1}\left(\tilde{H}_{\epsilon}, \mathfrak{g}\right)\right) .
$$

Note that $\left\{\tilde{X}_{\epsilon}: H \rightarrow \mathfrak{g}\right\}$ is a smooth family of functions. Now, since $H$ is compact, proof of vanishing of cohomology says that, for $k>0$, there exist an homotopy operator $h^{\epsilon}$ : 
$C_{\phi_{\epsilon}}^{k}\left(H_{\epsilon}, \mathfrak{g}\right) \rightarrow C_{\phi_{\epsilon}}^{k-1}(H, \mathfrak{g})$,

$$
h^{\epsilon}(c)\left(h_{1}, \ldots, h_{k-1}\right):=\int_{H_{\epsilon}} A d_{\left(\phi_{\epsilon}(h)\right)} c\left(h, h_{1}, \ldots, h_{k-1}\right) d h
$$

where the integral is computed by taking the Haar's measure defined on $H_{\epsilon}$. Then, since $\left\{\tilde{H}_{\epsilon}\right\}_{\epsilon}$ is a smooth family, the family of Haar's measures also varies smoothly, in the sense that $\int_{H_{\epsilon}} f$, for $f \in C^{\infty}(H)$, is a smooth function w.r.t. $\epsilon$. Hence, $\left\{u^{\epsilon}\right\}:=\left\{h^{\epsilon}\left(\tilde{X}_{\epsilon}\right)\right\}$ is a smooth family of elements in $\mathfrak{g}$. Thus,

$$
\tilde{X}_{\epsilon}=\delta_{\phi_{\epsilon}}\left(u^{\epsilon}\right)-\tilde{T}_{\epsilon}
$$

where $\tilde{T}_{\epsilon}=h^{\epsilon}\left(-\left(\phi_{\epsilon}\right)_{*} \xi_{\epsilon}\right) \in\left(\phi_{\epsilon}\right)_{*} C^{1}\left(\tilde{H}_{\epsilon}, \mathfrak{h}\right)$. So, define $T_{\epsilon} \in C^{1}\left(\tilde{H}_{\epsilon}, \mathfrak{h}\right)$ by $\tilde{T}_{\epsilon}=:\left(\phi_{\epsilon}\right)_{*} T_{\epsilon}$. By applying $\delta_{\phi_{\epsilon}}$, we get $-\xi_{\epsilon}=\delta_{\tilde{H}_{\epsilon}} T_{\epsilon}$. Then, by proposition (3.2.5), $\left\{T_{\epsilon}\right\}$ induces a smooth family of isomorphisms $F_{\epsilon}: \tilde{H}_{0} \rightarrow \tilde{H}_{\epsilon}$ with $F_{0}=I d_{H}$. Define the deformation of the inclusion $H \hookrightarrow G, \psi_{\epsilon}:=\phi_{\epsilon} \circ F_{\epsilon}$. We claim that $\left\{\psi_{\epsilon}\right\}_{\epsilon}$ is a trivial deformation. Indeed, its associated 1-cocycles, $\tilde{X}_{\epsilon}^{\prime}$, are

$$
\begin{aligned}
\tilde{X}_{\epsilon}^{\prime}(h) & =\tilde{X}_{\epsilon}\left(F_{\epsilon}(h)\right)+\left(\phi_{\epsilon}\right)_{*} T_{\epsilon}\left(F_{\epsilon}(h)\right) \\
& =\delta_{\phi_{\epsilon}}\left(u^{\epsilon}\right)_{\left(F_{\epsilon}(h)\right)}=\delta_{\phi_{\epsilon} \circ F_{\epsilon}}\left(u^{\epsilon}\right)_{(h)} \\
& =\delta_{\psi_{\epsilon}}\left(u^{\epsilon}\right)_{(h)} .
\end{aligned}
$$

Therefore, by proposition 3.2.13, the deformation $\left\{\psi_{\epsilon}\right\}$ is trivial. This means, $\phi_{\epsilon} \circ F_{\epsilon}=I_{g_{\epsilon}} \circ \iota$; obtaining thus the triviality of $\left\{H_{\epsilon}\right\}_{\epsilon}$.

Remark 3.2.17 In Coppersmith (1977) was already considered the question of rigidity of Lie subgroups, the author uses some analytic and algebraic tools to prove a more general notion of rigidity. Indeed, by assuming that the factor group $H / H^{0}$ is finitely generated (here $H^{0}$ is the connected component of $H$ at the identity), the rigidity result of Lie subgroups, as established in the previous theorem, can be seen as a particular case of Theorem 1 in Coppersmith (1977). Thus Theorem 3.2.16 above is implied by Theorem 1 in loc. cit., however our proof provides a simpler and more geometric proof of (our type of) rigidity but in turn it needs an additional condition of compactness on the statement.

Theorem 3.2.18 (Triviality of morphisms up to the action of Aut $(G)$ ) Let $\left\{\phi_{e}\right\}$ be a deformation of $\phi_{0}$ and $\tilde{X}_{\epsilon}$ its associated 1-cocycles. Assume that there exist $Z_{\epsilon} \in C^{1}(G, \mathfrak{g})_{\text {cl }}$ such that $\phi_{\epsilon}^{*} Z_{\epsilon}=\tilde{X}_{\epsilon}$ (i.e. $\left.Z_{\epsilon}\right|_{\phi_{\epsilon}(H)}=\tilde{X}_{\epsilon}$ ). If $\left\{r\left(Z_{\epsilon}\right)\right\}_{\epsilon}$ is a smooth and complete (time-dependent) vector field on $G$, then $\left\{\phi_{\epsilon}\right\}$ is trivial. 
Proof. This follows directly from the next fact,

$$
\begin{aligned}
\frac{d}{d \epsilon} \phi_{\epsilon}(h)=r_{\phi_{e}(h)}\left(\tilde{X}_{\epsilon}(h)\right) & =r_{\phi_{e}(h)}\left(Z_{\epsilon}\left(\phi_{\epsilon}(h)\right)\right) \\
& =\left(r\left(Z_{\epsilon}\right)\right)_{\left(\phi_{\epsilon}(h)\right)} .
\end{aligned}
$$

Thus $\left\{\epsilon \mapsto \phi_{\epsilon}(h)\right\}$ is an integral curve of $\left\{r\left(Z_{\epsilon}\right)\right\}_{\epsilon}$. So, if $\psi^{\epsilon_{2}, \epsilon_{1}}$ is the flow of $\left\{r\left(Z_{\epsilon}\right)\right\}, F_{\epsilon}:=\psi^{\epsilon, 0}$ are automorphisms of $G$ satisfying $F_{\epsilon}(\iota(h))=\phi_{\epsilon}(h)$. This is, $\left\{\phi_{\epsilon}\right\}$ is trivial up to the action of $\operatorname{Aut}(G)$. 


\section{Chapter 4}

\section{Deformation of symplectic groupoids}

The aim of this chapter is to introduce the study of deformation of geometries over Lie groupoids. In the case of symplectic structures, for instance, we can think of it as the deformation (over the same manifold) of both the groupoid and symplectic structures keeping a compatibility condition (the one reflecting the multiplicativity of the symplectic structure). In this sense, such a notion has a intuitively simple formulation (See Definition 4.2.1). We will work on this concept in an infinitesimal way by using the 'principle of deformation theory', that is, we get a cohomology theory controlling the deformations of symplectic groupoids, and use it to get an analogue of the classical Moser's theorem in symplectic geometry for the context of symplectic groupoids. For that, we first recall briefly some facts of VB-groupoids which will help us to understand the cohomology mentioned above.

\subsection{VB-groupoids}

Intuitively, a VB-groupoid can be thought of as a groupoid object in the category of vector bundles. They provide alternative ways to look at the representation theory and the deformation theory of Lie groupoids. For instance, the deformation complex of Lie groupoids (Section 2.1) can be seen as a complex naturally associated to the cotangent groupoid when regarded as a VB-groupoid, called the VB-complex (See Crainic et al. (2015a), del Hoyo and Ortiz (2016) and Remark 4.1.6 below). This point of view will be useful for us in following section. Thus we expose now briefly the notion of VB-groupoid, show some examples and describe the necessary tools about VB-groupoids which will be useful for us. For a more detailed description we refer the reader to (Mackenzie (2005a), Gracia-Saz and Mehta (2010b) and Bursztyn et al. (2016)).

Definition 4.1.1 A VB-groupoid $(\Gamma, E, \mathcal{G}, M)$ is a structure of two Lie groupoids and two 
vector bundles as in the diagram

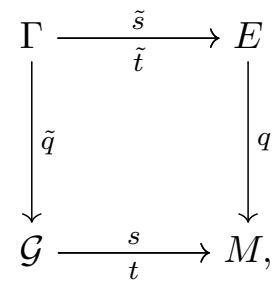

where the vertical directions are vector bundle structures and the horizontal ones are Lie groupoids, such that the structure maps of the groupoid $\Gamma$ (source, target, identity, multiplication, inversion) are vector bundle morphisms over the corresponding structure maps of the groupoid $\mathcal{G}$.

Remark 4.1.2 Note that the multiplication $m_{\Gamma}: \Gamma^{(2)} \longrightarrow \Gamma$ makes sense as a vector bundle morphism when it is considered the induced vector bundle structure of $\Gamma^{(2)}$ over $\mathcal{G}^{(2)}$ (guaranteed from the fact that the 'double source map' $(\tilde{q}, \tilde{s}): \Gamma \longrightarrow \mathcal{G}_{s} \times E$ is a surjective submersion (appendix A in Li-Bland and Ševera (2011)).

In this setting a morphism of VB-groupoids $\left(\phi_{\Gamma}, \phi_{E}, \phi_{\mathcal{G}}, \phi_{M}\right):(\Gamma, E, \mathcal{G}, M) \longrightarrow\left(\Gamma^{\prime}, E^{\prime}, \mathcal{G}^{\prime}, M^{\prime}\right)$ is a morphism $\left(\phi_{\Gamma}, \phi_{E}\right)$ between the Lie groupoids $\Gamma \rightrightarrows E$ and $\Gamma^{\prime} \rightrightarrows E^{\prime}$ preserving the vector bundle structures, i.e, such that $\phi_{\Gamma}$ and $\phi_{E}$ are vector bundle morphisms covering the maps $\phi_{\mathcal{G}}: \mathcal{G} \longrightarrow \mathcal{G}^{\prime}$ and $\phi_{M}: M \longrightarrow M^{\prime}$, respectively. Observe that, by restricting $\phi_{\Gamma}$ to the zero section, $\phi_{\mathcal{G}}$ turns out to be a Lie groupoid morphism.

Example 4.1.3 (Tangent VB-groupoid)

Given a Lie groupoid $\mathcal{G} \rightrightarrows M$ with source, target and multiplication maps $s, t$ and $m$, by applying the tangent functor one gets the tangent groupoid $T \mathcal{G} \rightrightarrows T M$ with structure maps $T s, T t, T m$ and so on. This tangent groupoid is further a VB-groupoid over $\mathcal{G} \rightrightarrows M$ (with respect to the tangent projections).

Remark 4.1.4 Note that in the previous example one has the following short exact sequences of vector bundles over $\mathcal{G}$,

$$
s^{*}\left(A_{\mathcal{G}}\right) \stackrel{-l \circ T i}{\longrightarrow} T \mathcal{G} \stackrel{(T t)^{!}}{\longrightarrow} t^{*}(T M)
$$

and

$$
t^{*}\left(A_{\mathcal{G}}\right) \stackrel{r}{\longrightarrow} T \mathcal{G} \stackrel{(T s) !}{\longrightarrow} s^{*}(T M)
$$

where $r$ and $l$ are the right and left multiplication on vector tangent to the $s$-fibers and $t$-fibers of $\mathcal{G}$, respectively; and $(T s)^{!}$and $(T t)^{!}$are the maps induced by $T$ s and $T t$ with image on the corresponding pullback bundles. 
Example 4.1.5 (Cotangent groupoid) As noticed in Coste et al. (1987), given a Lie groupoid $\mathcal{G}$ its cotangent bundle inherits a groupoid structure over the dual of the Lie algebroid of $\mathcal{G}$,

$$
T^{*} \mathcal{G} \rightrightarrows A_{\mathcal{G}}^{*}
$$

with source and target maps induced, respectively, from the dual of the exact sequences (4.2) and (4.3). Explicitly, for $\alpha_{g} \in T_{g}^{*} \mathcal{G}$ and $a \in \Gamma\left(A_{\mathcal{G}}\right)$,

$$
\left\langle\tilde{s}\left(\alpha_{g}\right), a_{s(g)}\right\rangle=-\left\langle\alpha_{g}, l_{g} \circ \operatorname{Ti}(a)\right\rangle
$$

and

$$
\left\langle\tilde{t}\left(\alpha_{g}\right), a_{t(g)}\right\rangle=\left\langle\alpha_{g}, r_{g}(a)\right\rangle .
$$

With multiplication determined by

$$
\left\langle\tilde{m}\left(\alpha_{g}, \beta_{h}\right), T m\left(v_{g}, w_{h}\right)\right\rangle=\left\langle\alpha_{g}, v_{g}\right\rangle+\left\langle\beta_{h}, w_{h}\right\rangle,
$$

for $\left(v_{g} \cdot w_{h}\right) \in(T \mathcal{G})^{(2)}$.

\subsubsection{VB-groupoid cohomology}

VB-groupoids have a special cohomology induced from their own groupoid structure which, additionally, takes into account the vector bundle structure. The corresponding complex is called the VB-groupoid complex and it was defined by Gracia-Saz and Mehta in Gracia-Saz and Mehta (2010b). It turns out to be isomorphic (non-canonically) to the deformation complex of Lie groupoids (Remark 4.1.6), providing another interpretation of the deformation complex of a groupoid; the definition is as follows.

Let $\Gamma$ be a VB-groupoid. The differentiable complex of $\Gamma$ (as Lie groupoid) has a natural subcomplex $C_{\text {lin }}^{*}(\Gamma)$ given by the fiberwise linear cochains of $\Gamma$. The VB-groupoid complex $C_{V B}^{*}(\Gamma)$ of $\Gamma$, is the subcomplex of $C_{\text {lin }}^{*}(\Gamma)$ determined by the left-projectabe elements of $C_{\text {lin }}^{*}(\Gamma)$, that is, the elements satisfying the following two conditions

1. $c\left(0_{g_{1}}, \gamma_{g_{2}}, \ldots, \gamma_{g_{k}}\right)=0$,

2. $c\left(0_{g} \cdot \gamma_{g_{1}}, \gamma_{g_{2}}, \ldots, \gamma_{g_{k}}\right)=c\left(\gamma_{g_{1}}, \gamma_{g_{2}}, \ldots, \gamma_{g_{k}}\right)$.

This complex has important applications in the theory of representations (up to homotopy) of Lie groupoids, in particular, it yields an interesting interpretation of the adjoint representation of a Lie groupoid, this latter turns out to be closely related to the complex of the tangent VB-groupoid (Gracia-Saz and Mehta (2010b)). But what is important for us, in this thesis, is the following notorious fact which concerns its relation with the deformation complex of Lie groupoids. 
Remark 4.1.6 A straightforward computation shows that the deformation complex of a Lie groupoid $\mathcal{G}$ is isomorphic to the VB-groupoid complex of its cotangent VB-groupoid, $C_{V B}^{*}\left(T^{*} \mathcal{G}\right)$ (del Hoyo and Ortiz (2016), Prop. 4.5). The isomorphism is given by $C_{d e f}^{*}(\mathcal{G}) \longrightarrow C_{V B}^{*}\left(T^{*} \mathcal{G}\right)$, $c \mapsto c^{\prime}$ with

$$
c^{\prime}\left(\eta_{g_{1}}, \ldots, \eta_{g_{k}}\right)=\left\langle\eta_{g_{1}}, c\left(g_{1}, \ldots, g_{k}\right)\right\rangle .
$$

\subsection{Deformations}

The notion of deformation of symplectic groupoids can be formulated in a natural way based on the the concept of deformation of Lie groupoids explained in Definition 2.1.2. We just add the condition of this latter deformation to be by symplectic groupoids.

Definition 4.2.1 (Strict deformations of symplectic groupoids)

Let $(\mathcal{G}, \omega)$ be a symplectic groupoid, $\left\{\mathcal{G}_{\epsilon}\right\}_{\epsilon}=\left\{\left(\mathcal{G}, \bar{m}_{\epsilon}\right)\right\}_{\epsilon}$ be a strict deformation of $\mathcal{G}$ and $\left\{\omega_{\epsilon} \in \Omega_{c l}^{2}(\mathcal{G})\right\}_{\epsilon}$ be a deformation of $\omega$ by symplectic forms. We say that the family $\left\{\left(\mathcal{G}_{\epsilon}, \omega_{\epsilon}\right)\right\}_{\epsilon}$ is a strict deformation of $(\mathcal{G}, \omega)$ if $\left\{\left(\mathcal{G}_{\epsilon}, \omega_{\epsilon}\right)\right\}_{\epsilon}$ is a family of symplectic groupoids. Analogously, by considering s-constant deformations of the Lie groupoid structure, it is defined an s-constant deformation of symplectic groupoids. The deformation such that $\left(\bar{m}_{\epsilon}, \omega_{\epsilon}\right)=(\bar{m}, \omega)$ for all $\epsilon$ will be called the constant deformation of $(\mathcal{G}, \omega)$.

Examples of deformations can be easily constructed (at least) in two simple ways.

Examples 4.2.2 Consider $(\mathcal{G}, \omega)$ a symplectic groupoid with $(M, \pi)$ the Poisson structure induced on the base $M$ of $\mathcal{G}$.

1. (Diffeomorphisms) Let $\left\{\phi_{\epsilon}: \mathcal{G} \longrightarrow \mathcal{G}\right\}$ be a family of diffeomorphisms with $\phi_{0}=I d_{\mathcal{G}}$. For every $\epsilon$, induce the groupoid structure $\mathcal{G}_{\epsilon}:=\left(\mathcal{G}, \bar{m}_{\epsilon}\right)$ in such a way that $\phi_{\epsilon}: \mathcal{G}_{\epsilon} \longrightarrow \mathcal{G}$ is an isomorphism. Thus $\left\{\mathcal{G}_{\epsilon}, \phi_{\epsilon}^{*} \omega\right\}_{\epsilon}$ turns out to be a deformation of $(\mathcal{G}, \omega)$.

2. (Gauge transformations) Let $\left\{\alpha_{\epsilon} \in \Omega_{c l}^{2}(M)\right\}_{\epsilon}$ be a smooth family of closed 2-forms on the base of $\mathcal{G}$ with $\alpha_{0}=0$ and assume that $\mathcal{G}$ is compact. The family $\left\{\left(\mathcal{G}, \Omega_{\alpha_{\epsilon}}\right)\right\}_{\epsilon}$, where $\Omega_{\alpha_{\epsilon}}=\omega+s^{*} \alpha_{\epsilon}-t^{*} \alpha_{\epsilon}$, is a deformation of $(\mathcal{G}, \omega)$, for $\epsilon$ small enough. Note in particular that $\left(\mathcal{G}, \Omega_{\alpha_{\epsilon}}\right)$ is the symplectic groupoid integrating the Poisson structure $\pi^{\alpha_{\epsilon}}$ on $M$ (the gauge transformation of $\pi$ by $\alpha_{\epsilon}$ ).

The previous two examples are just particular cases of what we call trivial deformations of $(\mathcal{G}, \omega)$. More generally, we have the following notion of equivalence of deformations.

Definition 4.2.3 (Equivalent deformations) Given two deformations $\left\{\left(\mathcal{G}_{\epsilon}, \omega_{\epsilon}\right)\right\}$ and $\left\{\left(\mathcal{G}_{\epsilon}^{\prime}, \omega_{\epsilon}^{\prime}\right)\right\}$ of the symplectic groupoid $(\mathcal{G}, \omega)$. We say that they are equivalent if there exists a smooth family of isomorphisms $\left\{F_{\epsilon}: \mathcal{G}_{\epsilon} \rightarrow \mathcal{G}_{\epsilon}^{\prime}\right\}$, with $F_{0}=I d_{\mathcal{G}}$, and a smooth family of 2-forms on $M$, 
$\left\{\alpha_{\epsilon}\right\}_{\epsilon}$, with $\alpha_{0}=0$, such that

$$
F_{\epsilon}^{*} \omega_{\epsilon}^{\prime}=\omega_{\epsilon}+\delta_{\epsilon}\left(\alpha_{\epsilon}\right), \text { for every } \epsilon \in I,
$$

where $\delta_{\epsilon}\left(\alpha_{\epsilon}\right)=s_{\epsilon}^{*}\left(\alpha_{\epsilon}\right)-t_{\epsilon}^{*}\left(\alpha_{\epsilon}\right)$. The deformation is said to be trivial if it is equivalent to the constant deformation.

\subsection{Deformation complex of (symplectic) groupoids}

In this section we revisit the deformation complex of Lie groupoids for the case of symplectic groupoids. If $(\mathcal{G}, \omega)$ is a symplectic groupoid we use the corresponding symplectic form to look at the deformation complex $C_{d e f}^{*}(\mathcal{G})$ as a subcomplex of $\left(\Omega^{1}\left(\mathcal{G}^{(*)}\right), \delta\right)$, the complex of 1 -forms on the nerve of $\mathcal{G}$ with differential coming from the simplicial structure of the nerve. Such a point of view will be of importance to get an infinitesimal interpretation of deformations of symplectic groupoids (the infinitesimal cocycles).

Consider the deformation complex $C_{d e f}^{*}(\mathcal{G})$ viewed as the VB-groupoid complex $C_{V B}^{*}\left(T^{*} \mathcal{G}\right)$ of $T^{*} \mathcal{G}$ (Remark 4.1.6). Denote by $\hat{C}^{*}\left(T^{*} \mathcal{G}\right)$ such a VB-complex. In particular, $\hat{C}^{*}\left(T^{*} \mathcal{G}\right) \subset$ $C_{\text {lin }}^{*}\left(T^{*} \mathcal{G}\right)$, then by using the groupoid isomorphism $\omega^{b}: T \mathcal{G} \rightarrow T^{*} \mathcal{G}$ (Lemma 3.6 in Bursztyn et al. (2009)), we get a subcomplex $\hat{C}^{*}(T \mathcal{G}) \subset C_{\text {lin }}^{*}(T \mathcal{G})$. Indeed, due to the fact that $\omega^{b}$ is also a vector bundle isomorphism, such a subcomplex consists in fact of linear cochains of $T \mathcal{G}$. Furthermore since, for each integer $k,(T \mathcal{G})^{(k)} \cong T \mathcal{G}^{(k)}$, the elements of $C_{\text {lin }}^{k}(T \mathcal{G})$ can be viewed as the fiberwise linear functions on $T \mathcal{G}^{(k)}$, i.e., as elements of $C_{\text {lin }}^{\infty}\left(T \mathcal{G}^{(k)}\right) \cong \Omega^{1}\left(\mathcal{G}^{(k)}\right)$. Also, this identification of $C_{\text {lin }}^{*}(T \mathcal{G})$ with $\Omega^{*}(\mathcal{G})$ is compatible with the differentials of the complexes involved. In fact, the differential in $C_{\text {lin }}^{*}(T \mathcal{G})$ is determined by the tangent prolongation of the structure maps of the nerve of $\mathcal{G}$ (it is the alternate sum of the dual of the face maps of the nerve of $T \mathcal{G})$, that is, by $T s, T t, T p r_{1}, T p r_{2}, T m, \ldots$, while the differential of $\Omega^{1}\left(\mathcal{G}^{(*)}\right)$ comes from the simplicial structure of the nerve of $\mathcal{G}$ as the alternate sum of the pullback of 1 -forms by the structure maps of the nerve of $\mathcal{G}$, so these two differentials reflect the two different ways to look at the pullback of 1 -forms by a function $f: M \longrightarrow N$ between two manifolds: as the usual pullback of forms $f^{*} \alpha$ and as the pullback of functions $(T f)^{*}: C_{l i n}^{\infty}(T N) \longrightarrow C_{l i n}^{\infty}(T M)$ by $T f$. With this setting, we denote by $\hat{\Omega}^{1}\left(\mathcal{G}^{(k)}\right)$ the subcomplex corresponding to $\hat{C}^{k}(T \mathcal{G})$ inside $\Omega^{1}\left(\mathcal{G}^{(k)}\right)$. In other words, we identify the deformation complex of $\mathcal{G}$ with a subcomplex of $\Omega^{1}\left(\mathcal{G}^{(*)}\right)$ :

Proposition 4.3.1 Let $(\mathcal{G}, \omega)$ be a symplectic groupoid and consider the cochain complex $\Omega^{1}\left(\mathcal{G}^{(*)}\right)$ of 1-forms on the nerve of $\mathcal{G}$. The deformation complex $C_{\text {def }}^{*}(\mathcal{G})$ of $\mathcal{G}$ is isomorphic to the subcomplex $\hat{\Omega}^{1}\left(\mathcal{G}^{(*)}\right)$ of $\Omega^{1}\left(\mathcal{G}^{(*)}\right)$.

Explicitly, under this identification, the complex $\hat{\Omega}^{1}(\mathcal{G})$ is completely described by translating the conditions of the VB-groupoid complex to $\Omega^{1}\left(\mathcal{G}^{(*)}\right)$. Indeed, let $\alpha \in \Omega^{1}\left(\mathcal{G}^{(k)}\right)$ be a 1-form on $\mathcal{G}^{(k)}$. We say that $\alpha$ is in $\hat{\Omega}^{1}\left(\mathcal{G}^{(k)}\right)$ if the following two conditions are satisfied. 
1. $\alpha \in \Gamma_{\mathcal{G}^{(k)}}\left(T^{*} \mathcal{G}^{(k)}\right)$ comes from a section $\hat{\alpha}$ of the bundle $p r_{1}^{*} T^{*} \mathcal{G} \rightarrow \mathcal{G}^{(k)}$, where $p r_{1}$ : $\mathcal{G}^{(k)} \rightarrow \mathcal{G}$ is the projection on the first coordinate. That is, there exists a necessarily unique section $\hat{\alpha}$ such that the left triangle below commutes

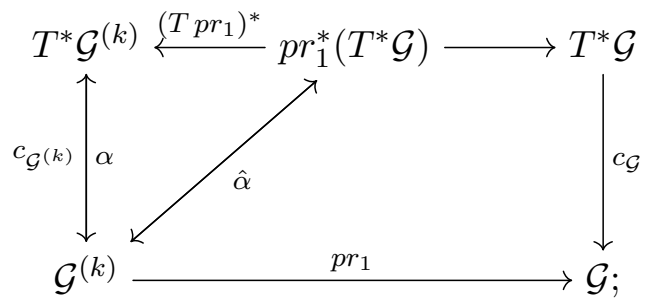

2. $\alpha$ is left-invariant by the action of $\mathcal{G}$ on $\mathcal{G}^{(k)}$ acting on the first coordinate.

In terms of elements, these identifications are

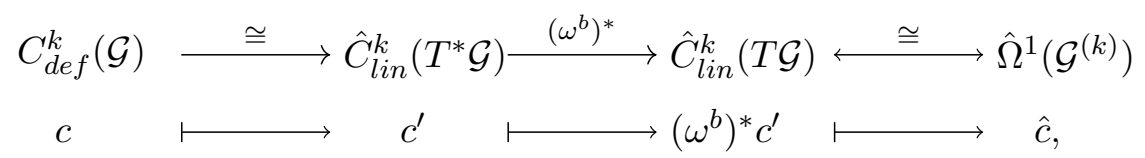

where $c^{\prime}\left(\eta_{g_{1}}, \ldots, \eta_{g_{k}}\right):=\left\langle\eta_{g_{1}}, c\left(g_{1}, \ldots, g_{k}\right)\right\rangle$ and, by construction,

$$
\begin{aligned}
\hat{c}\left(v_{g_{1}}, \ldots, v_{g_{k}}\right) & =\left(\omega^{b}\right)^{*} c^{\prime}\left(v_{g_{1}}, \ldots, v_{g_{k}}\right) \\
& =c^{\prime}\left(\omega^{b}\left(v_{g_{1}}\right), \ldots, \omega^{b}\left(v_{g_{k}}\right)\right) \\
& =-\left\langle\omega^{b}\left(c\left(g_{1}, \ldots, g_{k}\right)\right), v_{g_{1}}\right\rangle \\
& =-\left[\left(d p r_{1}\right)^{*}\left(\omega^{b}\left(c\left(g_{1}, \ldots, g_{k}\right)\right)\right)\right]\left(v_{g_{1}}, \ldots, v_{g_{k}}\right) .
\end{aligned}
$$

In other words, $c \in C_{d e f}^{k}(\mathcal{G})$ corresponds with the section $-\omega^{b} \circ c: \mathcal{G}^{(k)} \rightarrow p r_{1}^{*} T^{*} \mathcal{G}$ of condition (1) above. Condition (2) holds for $\hat{c}$ because it amounts to $c$ being $s$-projectable.

Thus, in particular, if $\left\{\left(\mathcal{G}_{\epsilon}, \omega_{\epsilon}\right)\right\}_{\epsilon}$ is an $s$-constant deformation of $(\mathcal{G}, \omega)$, under the identification above, the deformation cocycle $\xi_{0}$ of $\left\{\mathcal{G}_{\epsilon}\right\}_{\epsilon}$ corresponds to a 1-form $\zeta_{0} \in \Omega^{1}\left(\mathcal{G}^{(2)}\right)$. Observe also that for $X \in C_{d e f}^{1}(\mathcal{G})$, the corresponding 1-form on $\mathcal{G}$ is $-i_{X} \omega=-\omega^{b}(X)$.

Remark 4.3.2 Note that, in the identification above, we only use the multiplicativity and non-degeneracy conditions of $\omega$. Then, in particular, such an identification also holds for twisted symplectic groupoids.

\subsection{Associated cocycle and Moser's trick}

We now show the existing relation between deformations of symplectic groupoids and the Bott-Shulman complex, $\Omega^{*}\left(\mathcal{G}_{0}^{(*)}\right)$, which tells us how its total cohomology controls such 
deformations. Then we state a result analogue to the classical Moser's theorem in the context of symplectic groupoids. For simplicity we consider only $s$-constant deformations of symplectic groupoids.

Denote by $H_{B S}^{*}(\mathcal{G})$ the cohomology of the total complex associated to the Bott-Shulman double complex; this is the double complex $\left(\Omega^{q}\left(\mathcal{G}^{(p)}\right), \delta, d\right)$ whose horizontal differentials $\delta$ : $\Omega^{q}\left(\mathcal{G}^{(\bullet)}\right) \longrightarrow \Omega^{q}\left(\mathcal{G}^{(\bullet+1)}\right)$ are determined by the simplicial structure of the nerve of $\mathcal{G}$ and vertical differentials $d: \Omega^{\bullet}\left(\mathcal{G}^{(p)}\right) \longrightarrow \Omega^{\bullet+1}\left(\mathcal{G}^{(p)}\right)$ given by the de Rham differential of forms. For $\left\{\left(\mathcal{G}_{\epsilon}, \omega_{\epsilon}\right)\right\}$ a deformation of $(\mathcal{G}, \omega)$, we define the associated 3-cochain, $\eta_{0} \in \operatorname{Tot}^{3}\left(\Omega^{\bullet}\left(\mathcal{G}_{0}^{(\bullet)}\right)\right)$ by

$$
\eta_{0}:=\zeta_{0}-\dot{\omega}_{0} \in \Omega^{1}\left(\mathcal{G}^{(2)}\right) \oplus \Omega^{2}(\mathcal{G})
$$

Due to the following proposition, we also call the element $\eta_{0}$ the infinitesimal cocycle associated to the deformation $\left\{\left(\mathcal{G}_{\epsilon}, \omega_{\epsilon}\right)\right\}_{\epsilon}$.

Proposition 4.4.1 The 3-cochain $\eta_{0}:=\zeta_{0}-\dot{\omega}_{0} \in \operatorname{Tot}^{3}\left(\Omega^{*}\left(\mathcal{G}_{0}^{(*)}\right)\right)$, defined above, is a 3cocycle. The corresponding cohomology class in $H_{B S}^{3}\left(\mathcal{G}_{0}\right)$ depends only on the equivalence class of the deformation.

In order to prove this proposition we first need some lemmas:

Lemma 4.4.2 Denote by $\mathcal{G}^{[2]}$ the domain of the division map of $\mathcal{G}$, i.e., the space of pairs of arrows with the same source. The $k$-form $\omega \in \Omega^{k}(\mathcal{G})$ is multiplicative if, and only if,

$$
\bar{m}^{*} \omega=p_{1}^{*} \omega-p_{2}^{*} \omega
$$

where $p_{i}: \mathcal{G}^{[2]} \longrightarrow \mathcal{G}$ are the projections on $\mathcal{G}$ and $i: \mathcal{G} \longrightarrow \mathcal{G}$ is the inversion of $\mathcal{G}$

Proof. We just need to show that equation (4.5) is equivalent to equation

$$
m^{*} \omega=p r_{1}^{*} \omega+p r_{2}^{*} \omega
$$

where $p r_{i}: \mathcal{G}^{(2)} \longrightarrow \mathcal{G}$ are the projections from the space of composable arrows to $\mathcal{G}$. For this, we use the diffeomorphism $\psi: \mathcal{G}^{[2]} \longrightarrow \mathcal{G}^{(2)},(p, q) \longmapsto(p, i(q))$. Indeed, by applying $\psi^{*}$ to equation (4.6) and recalling that $i^{*} \omega=-\omega$ (Bursztyn et al. (2004), Lemma 3.1), we get equation (4.5).

Lemma 4.4.3 Let $\left\{\left(\mathcal{G}, \bar{m}_{\epsilon}\right)\right\}_{\epsilon \in I}$ be an s-constant deformation of $\mathcal{G}$. Define for every $\epsilon \in I$ the diffeomorphism $\bar{M}_{\epsilon}: \mathcal{G}^{[2]} \longrightarrow \mathcal{G}^{[2]},(p, q) \longmapsto\left(\bar{m}_{\epsilon}(p, q), i_{\epsilon}(q)\right)$ and the map $\psi_{\epsilon}: \mathcal{G}^{[2]} \longrightarrow$ $\mathcal{G}_{\epsilon}^{(2)},(p, q) \longmapsto\left(p, i_{\epsilon}(q)\right)$. These families of maps satisfy the following conditions:

1.

(i) $\bar{m}_{\epsilon} \circ \bar{M}_{\epsilon}^{-1}=p_{1}$

$$
=\left(p r_{1}\right)_{\epsilon} \circ \psi_{\epsilon}
$$


(ii) $p_{1} \circ \bar{M}_{\epsilon}^{-1}=m_{\epsilon} \circ \psi_{\epsilon}$;

$$
\text { (iii) } \begin{aligned}
p_{2} \circ \bar{M}_{\epsilon}^{-1} & =i_{\epsilon} \circ p_{2} \\
& =\left(p r_{2}\right)_{\epsilon} \circ \psi_{\epsilon} .
\end{aligned}
$$

2. If $\left\{V_{\epsilon}\right\}_{\epsilon}$ denotes the time-dependent vector field on $\mathcal{G}^{[2]}$ associated to the family of diffeomorphisms $\left\{\bar{M}_{\epsilon}\right\}_{\epsilon}$ (i.e., $\left.\frac{d}{d \tau}\right|_{\tau=\epsilon} \bar{M}_{\tau}(p, q)=V_{\epsilon}\left(\bar{M}_{\epsilon}(p, q)\right)$ ), then

$$
V_{\epsilon}(p, q)=\left(\xi_{\epsilon}\left(p, i_{\epsilon}(q)\right),\left.\frac{d}{d \tau}\right|_{\tau=\epsilon} i_{\tau}\left(i_{\epsilon}(q)\right)\right)
$$

\section{Proof.}

1. This is straightforward once that $\bar{M}_{\epsilon}^{-1}(p, q)=\left(m_{\epsilon}\left(p, i_{\epsilon}(q)\right), i_{\epsilon}(q)\right)$.

2. Note that $V_{\epsilon}(p, q)=\left.\frac{d}{d \tau}\right|_{\tau=\epsilon}\left(\bar{M}_{\tau} \circ \bar{M}_{\epsilon}^{-1}\right)(p, q)$, thus the proof follows by applying $\left.\frac{d}{d \tau}\right|_{\tau=\epsilon}$ to $\left(\bar{M}_{\tau} \circ \bar{M}_{\epsilon}^{-1}\right)(p, q)=\left(\bar{m}_{\tau}\left(m_{\epsilon}\left(p, i_{\epsilon}(q)\right), i_{\epsilon}(q)\right), i_{\tau}\left(i_{\epsilon}(q)\right)\right)$ (observe that such a derivative in the first component is exactly the groupoid deformation cocycle).

\section{Proof of proposition 4.4 .1}

The proof of the first part follows from differentiating the multiplicativity condition, as stated in equation (4.5), of the family $\left\{\omega_{\epsilon}\right\}_{\epsilon}$. In fact, by taking derivative with respect to $\epsilon$ and reordering, we get

$$
\left.\frac{d}{d \epsilon}\right|_{\epsilon=\lambda} \bar{m}_{\epsilon}^{*} \omega_{\epsilon}=\left(-\bar{m}_{\lambda}^{*}\left(\left.\frac{d}{d \epsilon}\right|_{\epsilon=\lambda} \omega_{\epsilon}\right)+p_{1}^{*}\left(\left.\frac{d}{d \epsilon}\right|_{\epsilon=\lambda} \omega_{\epsilon}\right)-p_{2}^{*}\left(\left.\frac{d}{d \epsilon}\right|_{\epsilon=\lambda} \omega_{\epsilon}\right)\right) .
$$

Now, to deal with the expression in the left hand side, note that if $\left\{\bar{M}_{\epsilon}: \mathcal{G}^{[2]} \longrightarrow \mathcal{G}^{[2]}\right\}_{\epsilon}$ is the family of diffeomorphisms defined in lemma 4.4 .3 by $\bar{M}_{\epsilon}(p, q)=\left(\bar{m}_{\epsilon}(p, q), i_{\epsilon}(q)\right)$, then $\left.\frac{d}{d \epsilon}\right|_{\epsilon=\lambda} \bar{m}_{\epsilon}^{*} \omega_{\lambda}$ is equal to $\left.\frac{d}{d \epsilon}\right|_{\epsilon=\lambda} \bar{M}_{\epsilon}^{*} p r_{1}^{*} \omega_{\lambda}$ which is equivalent to $\left.\frac{d}{d \epsilon}\right|_{\epsilon=\lambda} \bar{M}_{\lambda}^{*}\left(\bar{M}_{\epsilon} \circ \bar{M}_{\lambda}^{-1}\right)^{*} p r_{1}^{*} \omega_{\lambda}$. In this way, by making $\varphi^{\epsilon, \lambda}:=\bar{M}_{\epsilon} \circ \bar{M}_{\lambda}^{-1}$ (which, by construction of $\left\{V_{\epsilon}\right\}_{\epsilon}$, is its time-dependent (low), we have

$$
\begin{aligned}
\left.\frac{d}{d \epsilon}\right|_{\epsilon=\lambda} \bar{M}_{\lambda}^{*}\left(\bar{M}_{\epsilon} \circ \bar{M}_{\lambda}^{-1}\right)^{*} p_{1}^{*} \omega_{\lambda} & =\left.\bar{M}_{\lambda}^{*} \frac{d}{d \epsilon}\right|_{\epsilon=\lambda}\left(\varphi^{\epsilon, \lambda}\right)^{*} p_{1}^{*} \omega_{\lambda} \\
& =\bar{M}_{\lambda}^{*} L_{V_{\lambda}}\left(p_{1}^{*} \omega_{\lambda}\right)
\end{aligned}
$$

That is, by item (1) of lemma 4.4.3, equation (4.7) becomes 


$$
\begin{aligned}
L_{V_{\lambda}}\left(p_{1}^{*} \omega_{\lambda}\right) & =\left(\bar{M}_{\lambda}^{-1}\right)^{*}\left(-\bar{m}_{\lambda}^{*}\left(\left.\frac{d}{d \epsilon}\right|_{\epsilon=\lambda} \omega_{\epsilon}\right)+p_{1}^{*}\left(\left.\frac{d}{d \epsilon}\right|_{\epsilon=\lambda} \omega_{\epsilon}\right)-p_{2}^{*}\left(\left.\frac{d}{d \epsilon}\right|_{\epsilon=\lambda} \omega_{\epsilon}\right)\right) \\
& =-\psi_{\lambda}^{*}\left(p r_{1}\right)_{\lambda}^{*}\left(\left.\frac{d}{d \epsilon}\right|_{\epsilon=\lambda} \omega_{\epsilon}\right)+\psi_{\lambda}^{*} m_{\lambda}^{*}\left(\left.\frac{d}{d \epsilon}\right|_{\epsilon=\lambda} \omega_{\epsilon}\right)-\psi_{\lambda}^{*}\left(p r_{2}\right)_{\lambda}^{*}\left(\left.\frac{d}{d \epsilon}\right|_{\epsilon=\lambda} \omega_{\epsilon}\right) \\
& =-\psi_{\lambda}^{*} \delta^{\lambda}\left(\left.\frac{d}{d \epsilon}\right|_{\epsilon=\lambda} \omega_{\epsilon}\right) .
\end{aligned}
$$

Also, since $\omega_{\lambda}$ is a closed form, by Cartan's formula we have,

$$
\begin{aligned}
\left(\psi_{\lambda}^{-1}\right)^{*} L_{V_{\lambda}}\left(p_{1}^{*} \omega_{\lambda}\right) & \left.=d\left(\left(\psi_{\lambda}^{-1}\right)^{*}\left(V_{\lambda}\right\lrcorner p_{1}^{*} \omega_{\lambda}\right)\right) \\
& \left.=d\left[\left(\psi_{\lambda}\right)_{*} V_{\lambda}\right\lrcorner\left(\psi_{\lambda}^{-1}\right)^{*} p_{1}^{*} \omega_{\lambda}\right] \\
& \left.=d\left[\left(\psi_{\lambda}\right)_{*} V_{\lambda}\right\lrcorner\left(p r_{1}\right)_{\lambda}^{*} \omega_{\lambda}\right] \\
& =-d \zeta_{\lambda},
\end{aligned}
$$

where the last equality follows from identification (4.4) in Section 4.3. Namely,

$$
\begin{aligned}
\left.\left(\left(\psi_{\lambda}\right)_{*} V_{\lambda}\right\lrcorner\left(p r_{1}\right)_{\lambda}^{*} \omega_{\lambda}\right)_{(g, h)} & =\left.\left(d p r_{1}\right)_{\lambda}^{*}\right|_{(g, h)}\left[\left.\omega_{\lambda}^{b}\right|_{g}\left(d\left(p r_{1}\right)_{\lambda} \circ\left(d \psi_{\lambda}\right)_{\left(g, i_{\lambda}(h)\right)}\left(V_{\lambda}\left(g, i_{\lambda}(h)\right)\right)\right)\right] \\
& =\left.\left(d p r_{1}\right)_{\lambda}^{*}\right|_{(g, h)}\left[\left.\omega_{\lambda}^{b}\right|_{g}\left(\xi_{\lambda}(g, h)\right)\right] \\
& \left.=-\zeta_{\lambda}(g, h) \quad \text { by identification }(4.4)\right) .
\end{aligned}
$$

Therefore, we conclude that

$$
d \zeta_{\lambda}=\delta^{\lambda}\left(\left.\frac{d}{d \epsilon}\right|_{\epsilon=\lambda} \omega_{\epsilon}\right)
$$

This completes the first part by taking $\lambda=0$.

Take now $\left\{\left(\mathcal{G}_{\epsilon}^{\prime}, \omega_{\epsilon}^{\prime}\right)\right\}$ an equivalent deformation of $(\mathcal{G}, \omega)$, and denote by $\eta_{0}^{\prime}$ its infinitesimal cocycle. The exactness of $\eta_{0}-\eta_{0}^{\prime}$ follows from taking derivatives at $\epsilon=0$ of $F_{\epsilon}^{*} \omega_{\epsilon}^{\prime}=\omega_{\epsilon}+\delta_{\epsilon}\left(\alpha_{\epsilon}\right)$ (recall that $\left.\alpha_{0}=0\right)$. That is,

$$
F_{0}^{*}\left(d i_{Z_{0}} \omega_{0}^{\prime}+\dot{\omega}_{0}^{\prime}\right)=\dot{\omega}_{0}+\delta_{0}\left(\dot{\alpha}_{0}\right)
$$

where $\left\{Z_{\epsilon}\right\}_{\epsilon}$ is the time-dependent vector field associated to the family $\left\{F_{\epsilon}\right\}_{\epsilon}$. Thus,

$$
\dot{\omega}_{0}^{\prime}-\dot{\omega}_{0}=-d i_{Z_{0}} \omega_{0}^{\prime}+\delta_{0}\left(\dot{\alpha}_{0}\right)
$$

On the other hand, by considering the groupoid deformation cocycles, since every $F_{\epsilon}$ : $\mathcal{G}_{\epsilon} \rightarrow \mathcal{G}_{\epsilon}^{\prime}$ is an isomorphism for every $\epsilon, \xi_{0}^{\prime}-\xi_{0}=\delta_{0}^{\text {def }}\left(Z_{0}\right)$. Thus, in terms of forms, this 
amounts to $\zeta_{0}^{\prime}-\zeta_{0}=\delta_{0}\left(-i_{Z_{0}} \omega\right)$ (identification (4.4)).

Therefore, the equation above becomes

$$
\begin{aligned}
\eta_{0}-\eta_{0}^{\prime} & =-\dot{\omega}_{0}+\zeta_{0}-\left(-\dot{\omega}_{0}^{\prime}+\zeta_{0}^{\prime}\right) \\
& =-d i_{Z_{0}} \omega+\delta_{0}\left(\dot{\alpha}_{0}\right)+\delta_{0}\left(i_{Z_{0}} \omega\right) \\
& =D_{B S}^{0}\left(\dot{\alpha}_{0}+i_{Z_{0}} \omega\right)
\end{aligned}
$$

Corollary 4.4.4 The 3-cocycle associated to the trivial deformation vanishes in cohomology.

Remark 4.4.5 Note that, since a deformation $\left\{\left(\mathcal{G}_{\epsilon}, \omega_{\epsilon}\right)\right\}$ of $\left(\mathcal{G}_{0}, \omega_{0}\right)$ can be seen as a deformation of $\left(\mathcal{G}_{\lambda}, \omega_{\lambda}\right)$ for any $\lambda \in I$, the corresponding $\eta_{\lambda} \in T o t^{3}\left(\Omega^{*}\left(\mathcal{G}_{\lambda}^{(*)}\right)\right)$ is also a cocycle. Further, in the case of a trivial deformation we can say even more: every $\eta_{\lambda}$ is exact. In fact, this follows from noting that if $\left\{\left(\mathcal{G}_{\epsilon}, \omega_{\epsilon}\right)\right\}$ is a trivial deformation of $\left(\mathcal{G}_{0}, \omega_{0}\right),\left\{\left(\mathcal{G}_{\epsilon}, \omega_{\epsilon}\right)\right\}$ also can be regarded as a trivial deformation of $\left(\mathcal{G}_{\lambda}, \omega_{\lambda}\right)$ for any $\lambda \in I$.

The following theorem shows that, under appropriate regularity and compactness conditions, the converse statement of the previous remark is also true.

Theorem 4.4.6 (Triviality of deformations)

Let $\left\{\left(\mathcal{G}_{\epsilon}, \omega_{\epsilon}\right)\right\}_{\epsilon}$ be an s-constant deformation of a compact symplectic groupoid $(\mathcal{G}, \omega)$. If the family of cocycles $\eta_{\epsilon}:=\zeta_{\epsilon}-\dot{\omega}_{\epsilon}$ is transgressed by a smooth family of cochains, then $\left\{\left(\mathcal{G}_{\epsilon}, \omega_{\epsilon}\right)\right\}_{\epsilon}$ is a trivial deformation.

Proof. Since $\mathcal{G}$ is compact, $H_{\text {diff }}^{2}\left(\mathcal{G}_{\epsilon}\right)=0$ for all $\epsilon$, thus to say that the family $\left\{\eta_{\epsilon}\right\}_{\epsilon}$ is transgressed by a smooth family of 2 -cochains amounts to having

$$
\dot{\omega}_{\tau}-\zeta_{\tau}=D_{B S}^{\tau}\left(\chi_{\tau}+\tilde{\alpha}_{\tau}\right)
$$

with $\left\{\chi_{\tau} \in \Omega^{1}\left(\mathcal{G}_{\tau}\right)\right\}_{\tau}$ and $\left\{\tilde{\alpha}_{\tau} \in \Omega^{2}(M)\right\}_{\tau}$ smooth families of forms.

Then, we get $d \tilde{\alpha}_{\tau}=0, \dot{\omega}_{\tau}=-d\left(\chi_{\tau}\right)+\delta_{\tau}\left(\tilde{\alpha}_{\tau}\right)$ and $\delta_{s}\left(-\chi_{\tau}\right)=\zeta_{\tau}$, which amounts to $\delta_{\text {def }}^{\tau}\left(X_{\tau}\right)=\xi_{\tau}$, with $\chi_{\tau}=\iota_{X_{\tau}} \omega_{\tau}$. Thus, denoting by $\left(\phi_{\epsilon}, \varphi_{\epsilon}\right)$ the flow of the time-dependent field $\left\{X_{\epsilon}\right\}_{\epsilon}$ (starting at time zero), by taking $\hat{\alpha}_{\tau}:=\varphi_{\tau}^{*} \tilde{\alpha}_{\tau}$, and $\bar{\alpha}_{\tau}$ such that $\hat{\alpha}_{\tau}=\frac{d}{d \tau} \bar{\alpha}_{\tau}$, we get

$$
\dot{\omega}_{\tau}=-d\left(\iota_{X_{\tau}} \omega_{\tau}\right)+\delta_{\tau}\left(\left(\varphi_{\tau}^{-1}\right)^{*} \frac{d}{d s} \bar{\alpha}_{\tau}\right)
$$

which is,

$$
\left.\frac{d}{d \epsilon}\right|_{\epsilon=\tau}\left[\phi_{\epsilon}^{*} \omega_{\epsilon}-\delta_{0}\left(\bar{\alpha}_{\epsilon}\right)\right]=0
$$


Therefore,

$$
\phi_{\epsilon}^{*} \omega_{\epsilon}-\delta_{0}\left(\alpha_{\epsilon}\right)=\omega, \text { for } \alpha_{\epsilon}=\bar{\alpha}_{0}-\bar{\alpha}_{\epsilon} .
$$

That is, the deformation $\left\{\left(\bar{m}_{\epsilon}, \omega_{\epsilon}\right)\right\}$ is trivial.

Having in mind to get an analogue to the classical Moser's theorem in the context of symplectic groupoids, we now explain a variation of the concepts introduced in this chapter.

Note that, given a deformation $\left\{\left(\mathcal{G}_{\epsilon}, \omega_{\epsilon}\right)\right\}_{\epsilon}$ of $(\mathcal{G}, \omega)$, the family of elements $\eta_{\epsilon}=\zeta_{\epsilon}-\dot{\omega}_{\epsilon} \epsilon$ $\Omega^{1}\left(\mathcal{G}_{\epsilon}^{(2)}\right) \oplus \Omega^{2}\left(\mathcal{G}_{\epsilon}\right)$ is also a cocycle in the total complex of the double complex $B_{\epsilon}^{p, q}:=$ $\left(\Omega^{q}\left(\mathcal{G}_{\epsilon}^{p}\right)\right)_{q \geqslant 0, p \geqslant 1}$. More generally, we can consider this "new" total complex and, by analogous arguments to the ones exposed in this chapter, to develope an slight variation of the deformation theory of symplectic groupoids, which "forgets" of the base manifold of the groupoid $\mathcal{G}$ and yields a more closely related statement to the classical Moser's trick in symplectic geometry. Indeed, if we assume that trivial deformations of symplectic groupoids are (only) those deformations of the form given by the first item in example 4.2.2, and that two deformations are equivalent if the symplectic groupoids of the deformations are symplectomorphic by a smooth family of isomorphisms of the groupoids (that is, take $\alpha_{\epsilon}=0$ for all $\epsilon$ in definition 4.2.3) then we get the following groupoid-version of the classical Moser's trick.

Proposition 4.4.7 Let $\left\{\left(\mathcal{G}_{\epsilon}, \omega_{\epsilon}\right)\right\}_{\epsilon}$ be an s-constant deformation of a compact symplectic groupoid $(\mathcal{G}, \omega)$. Then, the family of cocycles $\eta_{\epsilon}:=\zeta_{\epsilon}-\dot{\omega}_{\epsilon} \in \operatorname{Tot}^{2}\left(B_{\epsilon}^{p, q}\right)$ is transgressed by a smooth family of cochains if and only if the deformation $\left\{\left(\mathcal{G}_{\epsilon}, \omega_{\epsilon}\right)\right\}_{\epsilon}$ is given by $\left(\phi_{\epsilon}\right)^{*} \omega_{\epsilon}=\omega_{0}$ for a smooth family of isomorphisms of groupoids $\phi_{\epsilon}:\left(\mathcal{G}, \bar{m}_{0}\right) \longrightarrow\left(\mathcal{G}, \bar{m}_{\epsilon}\right)$. 


\section{Chapter 5}

\section{Cohomological relation in a symplectic groupoid}

Let $(M, \pi)$ be a Poisson manifold and $\left(T^{*} M\right)_{\pi}$ its underlying Lie algebroid. In the context of deformation of Lie algebroids, Crainic and Moerdijk in (Crainic and Moerdijk (2004), section 4.4) define a map $i: H_{\pi}(M) \rightarrow H_{\text {def }}\left(\left(T^{*} M\right)_{\pi}\right)$ between the Poisson cohomology and the deformation cohomology of the algebroid $\left(T^{*} M\right)_{\pi}$, which are the cohomologies controlling deformations of Poisson structures and Lie algebroids respectively. In this sense, the main result of this chapter consists in the construction of a map $i_{\mathcal{G}}$ which can be thought of as the global counterpart of the map $i$. For that we first recall the notion of VB-algebroid and some related concepts which will be necessary to prove the main statements of this chapter. Also, we recall the deformation complex of Lie algebroids in order to review the map $i$.

\subsection{VB-algebroids}

Just as Lie algebroids are thought of as the infinitesimal counterpart of Lie groupoids, VB-groupoids have an infinitesimal analogue: VB-algebroids. These objects were defined by Mackenzie as $\mathcal{L} \mathcal{A}$-vector bundles in Mackenzie (1998) and then reinterpretated by Gracia-Saz and Mehta in Gracia-Saz and Mehta (2010a); and similar to the role of VB-groupoids for deformation and representation theories of Lie groupoids, VB-algebroids provide alternative interpretations of the deformation cohomology of Lie algebroids (see Remark 5.2.1) and of the representation theory of Lie algebroids Gracia-Saz and Mehta (2010a). Roughly speaking, VB-algebroids are vector bundles in the category of Lie algebroids. In this way, such a concept is supported in the more fundamental notion of double vector bundle (a vector bundle in the category of vector bundles), which we now proceed to define. 


\subsubsection{Double vector bundles}

In the same way that a vector bundle can be viewed as a Lie groupoid (with the addition map as the multiplication), a double vector bundle (DVB) can be regarded just as an example of a VB-groupoid (making the Lie groupoids involved in 4.1 to come from vector bundle structures). So, with this in mind, the following definition becomes natural.

Definition 5.1.1 A double vector bundle $(D, E, A, M)$, or simply $D$, is a commutative square of four vector bundles

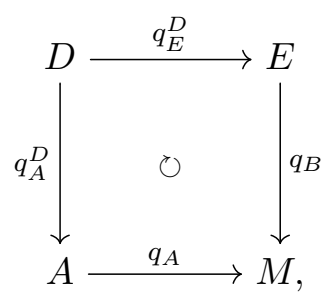

( $D$ has two vector bundle structures, on bases $E$ and $A$, which are themselves vector bundles over $M)$, such that $q_{E}^{D}$ and the addition map of $D \stackrel{q_{E}^{D}}{\longrightarrow} E,+_{E}: D \oplus_{E} D \longrightarrow D$, are vector bundle morphisms over $q_{A}$ and the addition of $A,+: A \oplus_{M} A \longrightarrow A$, respectively.

Remark 5.1.2 Making the conditions in definition above more explicit, one easily sees that $D$, as in diagram 5.1 , is a double vector bundle if

1. $q_{E}^{D}$ is a morphism of vector bundles,

2. $q_{A}^{D}$ is a morphism of vector bundles, and

3. $\left(d_{1}+{ }_{A} d_{3}\right)+{ }_{E}\left(d_{2}+{ }_{A} d_{4}\right)=\left(d_{1}+{ }_{E} d_{2}\right)+{ }_{A}\left(d_{3}+{ }_{E} d_{4}\right)$,

for quadruples $d_{1}, \ldots, d_{4} \in D$ such that $q_{E}^{D}\left(d_{1}\right)=q_{E}^{D}\left(d_{2}\right), q_{E}^{D}\left(d_{3}\right)=q_{E}^{D}\left(d_{4}\right), q_{A}^{D}\left(d_{1}\right)=q_{A}^{D}\left(d_{3}\right)$ and $q_{A}^{D}\left(d_{2}\right)=q_{A}^{D}\left(d_{4}\right)$. (Item (2) follows directly from the commutativity of $+_{E}$ with the bundle projections, and (3) expresses the linearity condition of the morphism $\left.+_{E}\right)$. The equation in (3) is also referred to as the interchange law.

In the vertical bundle structure on $D$ with base $A, \tilde{D}_{A}$, we denote by $\tilde{0}^{A}: A \longrightarrow D, a \longmapsto$ $\tilde{0}_{a}^{A}$ the zero-section; similarly, in the horizontal vector bundle structure on $D$ over $E, \tilde{D}_{E}$, we write $\tilde{0}^{E}: E \longrightarrow D, b \longmapsto \tilde{0}_{b}^{E}$. And the two vector bundles $A$ and $E$ over $M$, with zero-sections $0^{A}$ and $0^{E}$, respectively, are called the side bundles of (5.1).

Definition 5.1.3 (Morphisms of DVB)

$A$ morphism of double vector bundles $\left(\phi, \phi_{E}, \phi_{A}, \phi_{M}\right):(D, E, A, M) \longrightarrow\left(D^{\prime}, E^{\prime}, A^{\prime}, M^{\prime}\right)$ consists of maps $\phi: D \longrightarrow D^{\prime}, \phi_{E}: E \longrightarrow E^{\prime}, \phi_{A}: A \longrightarrow A^{\prime}, \phi_{M}: M \longrightarrow M^{\prime}$ such that each of $\left(\phi, \phi_{E}\right),\left(\phi, \phi_{A}\right),\left(\phi_{E}, \phi_{M}\right)$ and $\left(\phi_{A}, \phi_{M}\right)$ are morphisms of the corresponding vector 
bundles.

If $M=M^{\prime}, E=E^{\prime}$ and $\phi_{E}=i d_{E}$, one says that $\phi$ preserves $E$. If, further, $A=A^{\prime}$ and $\phi_{A}=i d_{A}$ one says that $\phi$ preserves the side bundles.

Besides the two side bundles of $D$, there is an important third vector bundle over $M$ related to $(D, E, A, M)$ : the core bundle, $C$, which is defined as the intersection of the kernels of $q_{E}^{D}$ and $q_{A}^{D}$. Thus, to avoid future confusions, when looking at $C$ as a vector subbundle of $D$, we denote an element $c \in C$ by $\bar{c}$. The core-bundle fits into the following exact sequence of vector bundles over $A$.

$$
0 \rightarrow q_{A}^{*} C \stackrel{\tau_{A}}{\longrightarrow} \tilde{D}_{A} \stackrel{\left(q_{E}^{D}\right)^{!}}{\longrightarrow} q_{A}^{*} E \rightarrow 0
$$

where $\left(q_{E}^{D}\right)^{!}$is the induced projection on $q_{A}^{*} E$ and $\tau_{A}(a, c)=\tilde{0}_{a}^{A}+{ }_{E} \bar{c}$ (observe that it makes sense because (i) $\tilde{0}_{a}^{A}$ and $\bar{c} \in D$ are in the same $q_{E}^{D}$-fiber over $0_{q_{A}(a)}^{E}$ and (ii) $q_{A}^{D}$ is a morphism, thus $\tau_{A}(a, c) \in \tilde{D}_{A}$ is over $\left.a \in A\right)$. This sequence is called the core sequence of $D$ over $A$. And analogously, there is a core sequence of $D$ over $E$.

One interesting thing about these core sequences is that, for instance in (5.2), a section of $C$ induces a section in $\Gamma_{A}(D)$ of $\tilde{D}_{A}$. In fact, $c \in \Gamma(C)$ defines $c^{A} \in \Gamma_{A}(D)$ by

$$
c^{A}(a):=\tau\left(a, c_{q_{A}(a)}\right)=\tilde{0}_{a}^{A}+{ }_{E} \bar{c}_{q_{A}(a)} .
$$

The section $c^{A}$ is called the core section over $A$ corresponding to $c$. And analogously, with the core sequence over $E$, one gets core sections over $E$.

Example 5.1.4 (Tangent prolongation double vector bundle)

1. Consider the vector bundle $(E \stackrel{q}{\longrightarrow} M)$. Applying the tangent functor to the structure maps of $E$ (projection, addition, zero-section) yields the DVB

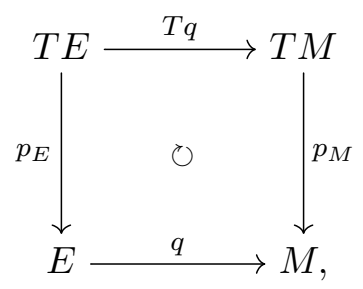

with core $E$. The core sections over $E$ and $T M$ corresponding to $\alpha \in \Gamma(E)$ are respectively:

$\alpha^{\uparrow} \in \Gamma_{E}(T E): \quad \alpha^{\uparrow}(e)=\tilde{0}_{e}^{E}+T M \overline{\alpha\left(p_{E}(e)\right)}=\left.\frac{d}{d \epsilon}\right|_{\epsilon=0}\left(e+\epsilon \alpha\left(p_{E}(e)\right)\right)$ (the vertical lift of $\left.\alpha\right) ;$

and

$$
\hat{\alpha} \in \Gamma_{T M}(T E), \quad \hat{\alpha}\left(v_{x}\right)=\tilde{0}_{v_{x}}^{T M}+_{E} \overline{\alpha(x)} \in T_{0_{x}^{E}} E .
$$


Note that in this context $\tilde{0}^{T M}=T\left(0^{E}\right)$, and $\overline{\alpha(x)}=\alpha^{\uparrow}\left(0_{x}^{E}\right)$.

2. Consider $F: E \longrightarrow E^{\prime}$ a morphism of vector bundles over $f: M \longrightarrow M^{\prime}$. The tangent prolongation of $F$ induces the morphism of vector bundles $(T F, T f, F, f): T E \longrightarrow T E^{\prime}$.

Example 5.1.5 (Dual DVB) By dualizing the core exact sequence over A (5.2), we can induce a double vector bundle

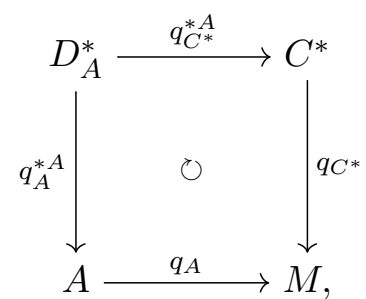

with core $E^{*}$, where $D_{A}^{*}$ denotes the dual over $A$, and $q_{A}^{* A}: D_{A}^{*} \longrightarrow C^{*}$ comes from the dual of $\tau_{A}$ :

$$
\left\langle q_{C^{*}}^{* A}\left(\eta_{a}\right), c\right\rangle=\left\langle\eta_{a}, \tau_{A}(a, c)\right\rangle
$$

for $\eta_{a}:\left(q_{A}^{D}\right)^{-1}(a) \stackrel{\text { linear }}{\longrightarrow} \mathbb{R}$ and $c \in C_{q_{A}(a)}$. The addition map $+_{C^{*}}: D_{A}^{*} \oplus_{C^{*}} D_{A}^{*} \longrightarrow D_{A}^{*}$ is defined in such a way that the natural pairing $\langle\rangle:, D_{A}^{*} \oplus \tilde{D}_{A} \longrightarrow \mathbb{R}$ is linear with respect to the vector bundle structure over $C^{*} \oplus_{M} E$, i.e.,

$$
\left\langle\eta_{a}+C^{*} \eta_{a^{\prime}}^{\prime}, d_{a}+{ }_{E} d_{a^{\prime}}^{\prime}\right\rangle=\left\langle\eta_{a}, d_{a}\right\rangle+\left\langle\eta_{a^{\prime}}^{\prime}, d_{a^{\prime}}^{\prime}\right\rangle
$$

Note that $\eta_{a}+C^{*} \eta_{a}^{\prime}$ is determined by the expression above due to the fact that any element in $\left(\tilde{D}_{A}\right)_{a+a^{\prime}}$ can be written as the sum of elements $d \in\left(\tilde{D}_{A}\right)_{a}$ and $d^{\prime} \in\left(\tilde{D}_{A}\right)_{a^{\prime}}$. It is not hard to see that $+_{C^{*}}$ given in this form is well-defined. The zero above $\kappa \in C_{x}^{*}$, denoted by $\tilde{0}_{\kappa}^{* A}$ $\left(\tilde{0}_{\kappa}^{* A}:\left(\tilde{D}_{A}\right)_{0_{x}^{A}}=\left[\operatorname{Ker}\left(q_{A}^{D}\right) !\right]_{0_{x}^{A}} \stackrel{\text { linear }}{\longrightarrow} \mathbb{R}\right)$, is defined by

$$
\left\langle\tilde{0}_{\kappa}^{* A}, \tilde{0}_{e}^{E}+{ }_{A} \bar{c}\right\rangle=\langle\kappa, c\rangle, \text { for } e \in E_{x}, c \in C_{x}
$$

Analogously, one can take the dual of the core exact sequence over E, inducing a DVB $\left(D_{E}^{*}, E, C^{*}, M\right)$ with core $A^{*}$. See Mackenzie (2005a) or Mackenzie (2005b), for further details.

Remark 5.1.6 We now note that, similarly to the dual of a morphism of vector bundles over the same base covering the identity, it is defined a dual of a morphism of DVBs which have one same side bundle. If $\left(\phi, \phi_{E}, i d_{A}, \phi_{M}\right):(D, E, A, M) \longrightarrow\left(D^{\prime}, E^{\prime}, A, M\right)$ is a morphism of DVB preserving $A$, by dualizing $\phi$ as a morphism of vector bundles over $A$, one obtains a morphism of double vector bundles preserving $A\left(\phi_{A}^{*}, \phi_{C}^{*}, i d_{A}, \phi_{M}\right):\left(D_{A}^{\prime *},\left(C^{\prime}\right)^{*}, A, M\right) \longrightarrow$ $\left(D_{A}^{*}, C^{*}, A, M\right)$ with core morphism $\phi_{E}^{*}:\left(E^{\prime}\right)^{*} \longrightarrow E^{*}$. 


\subsubsection{Isomorphisms of duals of DVBs}

As example 5.1.5 shows, there are two different ways to dualize a DVB; one vertical dualization and another one horizontal. An interesting fact is that the horizontal dual of the dual DVB (5.3), $\left(\left(D_{A}^{*}\right)_{C^{*}}^{*}, C^{*}, E, M\right)$, turns out to be a DVB with the same side bundles and core as $\left(D_{E}^{*}, E, C^{*}, M\right)$. In fact, they are isomorphic double vector bundles. Such an isomorphism, $Z_{E}: D_{E}^{*} \longrightarrow\left(D_{A}^{*}\right)_{C^{*}}^{*}$, is the one induced by a natural pairing, $|\cdot, \cdot|$, existing between the vertical and horizontal duals $D_{A}^{*}$ and $D_{E}^{*}$ as vector bundles over $C^{*}$,

$$
\left|\eta_{a}, \theta_{e}\right|=\left\langle\eta_{a}, d\right\rangle_{A}-\left\langle\theta_{e}, d\right\rangle_{E}
$$

$\eta_{a} \in D_{A}^{*}, \theta_{e} \in D_{E}^{*}$ with $q_{C^{*}}^{* A}\left(\eta_{a}\right)=q_{C^{*}}^{* E}\left(\theta_{e}\right)$ and $d \in D$ any element such that the canonical pairings in the RHS make sense.

Of course, this pairing also yields the isomorphism $D_{A}^{*} \stackrel{Z_{A}}{\cong}\left(D_{E}^{*}\right)_{C^{*}}^{*}$, which induces the identity on the core bundles $E^{*}$ and on the sides $C^{*}$, and is $-i d_{A}$ on the sides $A$, (Mackenzie (2005a), Corollary 9.2.4). This shows that taking the dual over $C^{*}$ interchanges the vertical and horizontal duals of $(D, E, A, M)$. Or equivalently, that alternation of vertical and horizontal duals interchanges the duals (e.g. horizontal dual followed by vertical one is the (flip of the) vertical one).

\subsubsection{Reversal isomorphism}

Examples 5.1.4 and 5.1.5 show us two important ways to get double vector bundles (by tangent prolongation and dualizing). In fact, by considering dualization of (honest) vector bundles also, there exists a certain compatibility between these processes: dualization commutes with tangent prolongation (up to a canonical isomorphism). Indeed, the tangent lift of the canonical pairing, $\langle,\rangle_{A}$, between $A$ and $A^{*}$ over $M$ induces the tangent pairing $\langle\langle,\rangle\rangle_{A}$ between $T A$ and $T\left(A^{*}\right)$ over $T M-$ for $\left(v_{a^{*}}, w_{a}\right) \in T A^{*} \oplus_{T M} T A,\left\langle\left\langle v_{a^{*}}, w_{a}\right\rangle\right\rangle_{A}:=\left.\frac{d}{d \epsilon}\right|_{\epsilon=0}\langle\gamma(\epsilon), \alpha(\epsilon)\rangle_{A}$ where $\gamma(\epsilon)$ and $\alpha(\epsilon)$ are curves on $A^{*}$ and $A$ determining $v_{a^{*}}$ and $w_{a}$, respectively, with $p_{A^{*}}(\gamma(\epsilon))=p_{A}(\alpha(\epsilon))$ - yielding the isomorphism $I_{A}: T A^{*} \longrightarrow(T A)_{T M}^{*}$, which is also an isomorphism of DVBs, between the tangent prolongation of the dual of $A$ and the (horizontal) dual of the tangent prolongation of $A$. This isomorphism, $I_{A}$, preserves the sides and core bundles, and is often called the internalization map (Mackenzie (2005a), Prop. 9.3.2).

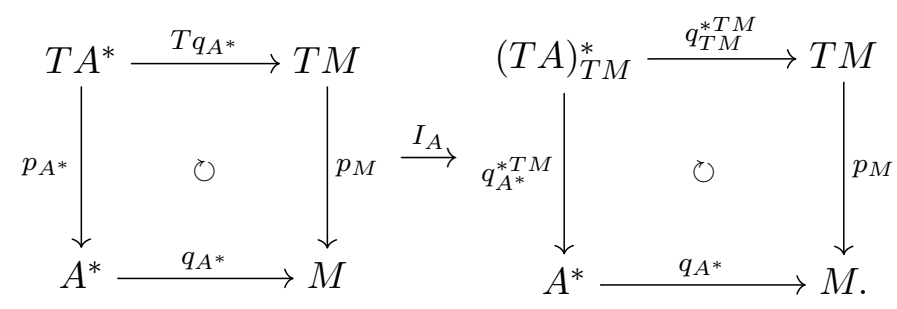


Consider now the tangent prolongation DVB of $A \longrightarrow M$. The natural pairing existing between its (horizontal and vertical) duals induces the isomorphism

$$
Z_{A}: T^{*} A=(T A)_{A}^{*} \longrightarrow\left((T A)_{T M}^{*}\right)_{A^{*}}^{*}
$$

Composing with the dual of $I_{A}$ over $A^{*}$ the following isomorphism of DVBs is obtained

$$
\left(I_{A}\right)_{A^{*}}^{*} \circ Z_{A}: T^{*} A \longrightarrow T^{*} A^{*}
$$

which (like $Z_{A}$ ) induces $-i d_{A}$ on the side bundles $A$ and preserves the cores, $T^{*} M$, and sides, $A^{*}$. Therefore (to preserve the side bundles) it is defined the map

$$
R_{A}=\left(\left(I_{A}\right)_{A^{*}}^{*} \circ Z_{A}\right)^{-1} \circ\left(-A^{*} i d_{T^{*} A^{*}}\right): T^{*} A^{*} \longrightarrow T^{*} A,
$$

which is an isomorphisms of DVBs preserving the sides and inducing $-i d_{T^{*} M}$ on the cores. The map $R_{A}$ is called the reversal isomorphism.

Alternatively, it is possible to describe this map in a simple way by using local coordinates. If $\left(x^{i}, \zeta_{d}\right)$ are (fibered) local coordinates of $A^{*} \longrightarrow M$, one writes the coordinates of $T^{*} A^{*}$ as $\left(x^{i}, \zeta_{d}, p_{i}, u^{d}\right)$, where $\left(p_{i}\right)$ and $\left(u^{d}\right)$ describe, respectively, a point in $T_{x}^{*} M$ and in $A_{x}$ (the dual of the tangent space to the fiber of $A^{*} \longrightarrow M$ over $\left.x\right)$. And analogously, $T^{*} A$ is described locally by the coordinates $\left(x^{i}, u^{d}, p_{i}, \zeta_{d}\right)$. In this coordinates $R_{A}$ has the form

$$
R_{A}\left(x^{i}, \zeta_{d}, p_{i}, u^{d}\right)=\left(x^{i}, u^{d},-p_{i}, \zeta_{d}\right)
$$

Note that if we would define $R_{A}$ locally by (5.5), it is easy to see that $R_{A}$ glues well to determine the reversal isomorphism globally, on $T^{*} A^{*}$.

The following propositions tell us about the naturality properties that the several previous maps satisfy. Before that, for clarity of the statements, we make a convention about the dual of morphisms of vector bundles and DVBs.

Remark 5.1.7 Consider $A$ and $B$ two vector bundles over $M_{1}$ and $M_{2}$, respectively, and $\Phi: A \longrightarrow B$ a morphism covering the diffeomorphism $f: M_{1} \longrightarrow M_{2}$. In this context, there is a natural induced isomorphism (the pointwise dual of $\Phi$ ) between the duals of $B$ and $A$ covering $f^{-1}$ which we denote by $\Phi_{f^{-1}}^{\star}: B^{*} \longrightarrow A^{*}$. And analogously, we can consider the dual of a morphism of double vector bundles which have one isomorphic side bundle. For instance, if $f: A \longrightarrow B$ is an isomorphism, we have the dual of the DVB morphism $T f: T A \longrightarrow T B$ over the isomorphism $f$, denoted by $(T f)_{f^{-1}}^{\star}: T^{*} B \longrightarrow T^{*} A$.

Proposition 5.1.8 Consider $A$ and $B$ two vector bundles over $M$.

1. $I_{A}$ is compatible with morphisms of vector bundles: If $f: A \longrightarrow B$ is a morphism of vector bundles covering the identity, the internalization maps make the following 
diagram commute

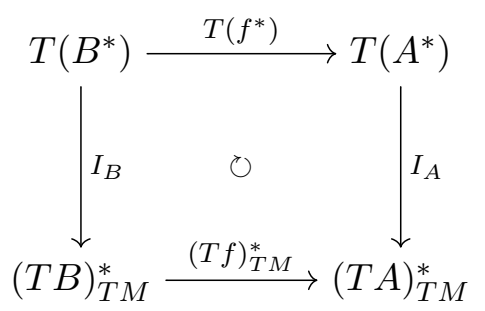

2. $Z_{A}$ is compatible with isomorphisms of vector bundles: If $f: A \longrightarrow B$ is an isomorphism of vector bundles, we have the following commutative diagram

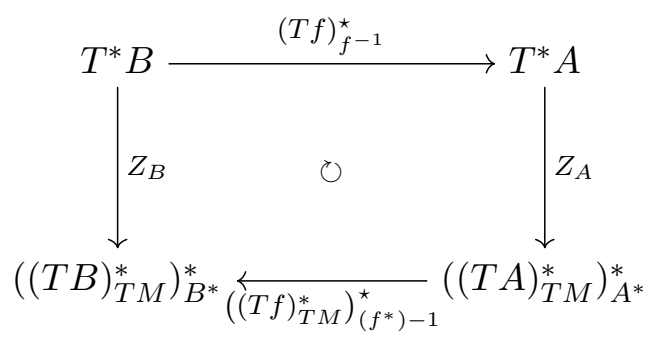

\section{Proof.}

1. Follows from looking at the corresponding tangent pairings. Note that, if $v_{b^{*}} \in T_{b^{*}} B^{*}$,

$$
I_{A}\left(T f^{*}\left(v_{b^{*}}\right)\right)=\left\langle\left\langle T f^{*}\left(v_{b^{*}}\right), \cdot\right\rangle\right\rangle_{A}:(T A)_{T M} \stackrel{\text { linear }}{\longrightarrow} \mathbb{R},
$$

where $(T A)_{T M}$ refers to the vector bundle structure of $T A$ over $T M$. Then, if $v_{b^{*}}=$ $\left.\frac{d}{d \epsilon}\right|_{\epsilon=0} \gamma(\epsilon)$ and $X_{a}=\left.\frac{d}{d \epsilon}\right|_{\epsilon=0} \alpha(\epsilon) \in T_{a} A$,

$$
\begin{aligned}
I_{A}\left(T f^{*}\left(v_{b^{*}}\right)\right)\left(X_{a}\right) & =\left.\frac{d}{d \epsilon}\right|_{\epsilon=0}\left\langle f^{*}(\gamma(\epsilon)), \alpha(\epsilon)\right\rangle_{A} \\
& =\left.\frac{d}{d \epsilon}\right|_{\epsilon=0}\langle\gamma(\epsilon), f(\alpha(\epsilon))\rangle_{B} \\
& =\left\langle\left\langle v_{b^{*}}, T f\left(X_{a}\right)\right\rangle\right\rangle_{B} \\
& =\left\langle\left\langle v_{b^{*}}, \cdot\right\rangle\right\rangle_{B}\left(T f\left(X_{a}\right)\right) \\
& =\left[(T f)_{T M}^{*}\left(I_{B}\left(v_{b^{*}}\right)\right)\right]\left(X_{a}\right)
\end{aligned}
$$

2. It suffices to compare the pairings introduced in subsection 5.1.2: $|\cdot, \cdot|_{A}: T^{*} A \oplus_{T M}$ $(T A)_{T M}^{*} \longrightarrow \mathbb{R}$ and $|\cdot, \cdot|_{B}: T^{*} B \oplus_{T M}(T B)_{T M}^{*} \longrightarrow \mathbb{R}$. In fact, commutativity of (5.7) amounts to have $\left|(T f)_{f^{-1}}^{\star}(\beta),(T f)_{T M}^{*}\left(\mathfrak{F}_{B}\right)\right|_{A}=\left|\beta, \mathfrak{F}_{B}\right|_{B}$, for $\left(\beta, \mathfrak{F}_{B}\right) \in T^{*} B \oplus_{T M}$ 
$(T B)_{T M}^{*}$. And this follows from noting that

$$
\begin{aligned}
\left|(T f)_{f^{-1}}^{\star}(\beta),(T f)_{T M}^{*}\left(\mathfrak{F}_{B}\right)\right|_{A} & =\left\langle(T f)_{f^{-1}}^{\star}(\beta), v\right\rangle_{A}-\left\langle(T f)_{T M}^{*}\left(\mathfrak{F}_{B}\right), v\right\rangle_{T M} \\
& =\langle\beta, T f(v)\rangle_{B}-\left\langle\mathfrak{F}_{B}, T f(v)\right\rangle_{T M} \\
& =\left|\beta, \mathfrak{F}_{B}\right|_{B}
\end{aligned}
$$

for $v \in T A$ such that the relevant pairings are well defined.

The map $R_{A}$ also satisfies several interesting properties (e.g. it is an antisymplectomorphism with respect to the canonical symplectic structures on the cotangent bundles (Mackenzie (2005a), Thm 9.5.2)), we remark some of them which will be useful for later use in this chapter. The first one, which is direct by using the previous proposition, describes the naturality of $R_{A}$ with respect to isomorphisms of vector bundles. Another property is in Proposition 5.1.15 below.

Proposition 5.1.9 Consider $A$ and $B$ two vector bundles over $M$. If $f: A \longrightarrow B$ is an isomorphism covering the identity, the following diagram commutes

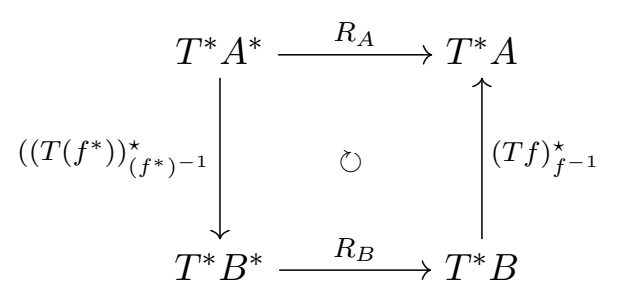

Proof. The commutativity follows from previous proposition by dualizing the internalization maps, indeed we get

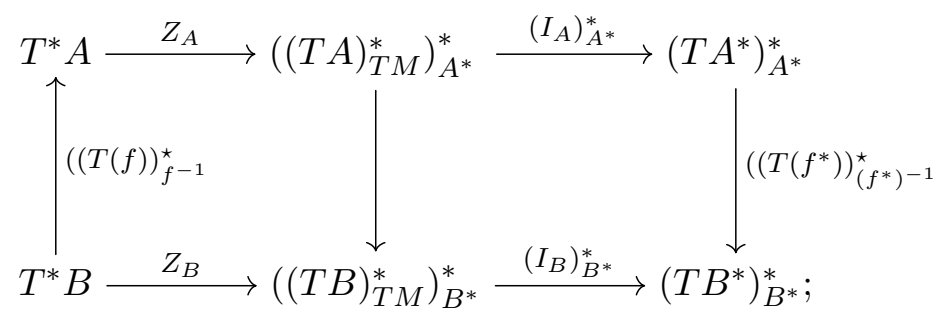

which implies diagram (5.8) (right vertical map is a DVB morphism, therefore commutes with scalar multiplication by -1$)$.

\subsubsection{Linear sections}

Consider a DVB as in (5.1). Beside the core sections of $D$ over $A, \Gamma_{c}(D, A)$, induced from the core exact sequence (5.2), there is another special type of sections of $\tilde{D}_{A}$, called the linear 
sections of $D$ over $A$, whose set is denoted by $\Gamma_{l}(D, A)$.

Definition 5.1.10 A section $X \in \Gamma(D, A)$ is called linear if it is a vector bundle morphism from $A \longrightarrow M$ to $D \longrightarrow E$.

Example 5.1.11 In example 5.1.4, if $X \in \Gamma(E)$ is a section of $E$, its tangent prolongation $T(X):=d(X) \in \Gamma(T E, T M)$ is a linear section of $T E$ over $T M$. Linear sections of $T E$ over $E$ are called linear vector fields of $E$.

An interesting fact about linear and core sections is that they span the other sections of $D$ over $A$ Mackenzie (2011). This follows essentially from taking a special splitting of the core exact sequence (5.2). Note first that if $\sigma:\left(q_{A}^{D}\right)^{*} E \longrightarrow \tilde{D}_{A}$ is a splitting of (5.2) and $\beta \in \Gamma(E, M)$ is a section of $E$, then $\sigma \circ\left(q_{A}^{*} \beta\right)$ is a linear section of $D$ over $A$ if, and only if, $\sigma$ is (also) linear when viewed as a morphism of vector bundles over the base $E$; that is, if and only if $\sigma$ is a splitting in the setting of double vector bundles. The local existence of such splittings is guaranteed by using the local form of DVB (over open neighborhoods in $M$ ) showed by Grabowski and Rotkiewicz Grabowski and Rotkiewicz (2009), and a partition of unity argument proves that such a splitting exists globally. With this in mind, if $X \in \Gamma(D, A)$ is any section of $D$ over $A, Y=X-{ }_{A} \sigma\left(\left(q_{E}^{D}\right)^{!} X\right)$ is a section of $A$ which projects to zero by $\left(q_{E}^{D}\right)^{!}$, thus $Y=\tau_{A}(\tilde{Y})$, where $\tilde{Y} \in \Gamma_{A}\left(q_{A}^{*} C\right) \cong C^{\infty}(A) \otimes_{C^{\infty}(M)} \Gamma(C)$, implying that $Y$ is a sum $\Sigma_{i} F_{i} \cdot Y_{i}^{A}$, for $F_{i} \in C^{\infty}(A)$ and $Y_{i}^{A}$ the core sections corresponding to some sections $Y_{i} \in \Gamma(C)$. In this way, by using this fact, we can simplify some statements in terms of sections of $D$ over $A$ by taking only linear and core sections, see for instance Example 5.1.13 below.

\subsubsection{VB-algebroids}

Definition 5.1.12 A VB-algebroid is a DVB as in (5.1), where the vector bundle $D \longrightarrow E$ is a Lie algebroid with anchor map $\rho_{D}: D \longrightarrow T E$ being a vector bundle morphism over $A \longrightarrow T M$ and such that the Lie bracket $[\cdot, \cdot]_{D}$ satisfies the following conditions:

1. $\left[\Gamma_{l}(D, E), \Gamma_{l}(D, E)\right]_{D} \subset \Gamma_{l}(D, E)$,

2. $\left[\Gamma_{l}(D, E), \Gamma_{c}(D, E)\right]_{D} \subset \Gamma_{c}(D, E)$,

3. $\left[\Gamma_{l}(D, E), \Gamma_{l}(D, E)\right]_{D}=0$.

As pointed out by Gracia-Saz and Mehta in Gracia-Saz and Mehta (2010a), a VB-algebroid $D \longrightarrow E$ induces a Lie algebroid structure on $A \longrightarrow M$ and the structure maps (projection, zero section, sum) of the vertical bundle structures form Lie algebroid morphisms. In this sense, a VB-algebroid can be though as a vector bundle in the category of Lie algebroids, (Gracia-Saz and Mehta (2010a), Thm. 3.7). 
Example 5.1.13 (Tangent Lie algebroid)

Given $A \stackrel{\pi}{\longrightarrow} M$ a Lie algebroid, there is a Lie algebroid structure on the tangent prolongation $T A \stackrel{T \pi}{\longrightarrow} T M$ in such a way that the tangent $D V B$ in example 5.1 .4 becomes a $V B$-algebroid. Denoting by $\hat{X}_{i} \in \Gamma_{c}(T A, T M), i=1,2$, the core section corresponding to $X_{i} \in \Gamma(A)$, the Lie bracket is defined by:

$$
\left[T X_{1}, T X_{2}\right]=T\left[X_{1}, X_{2}\right],\left[T X_{1}, \hat{X}_{2}\right]=\left[X_{1}, X_{2}\right],\left[\hat{X}_{1}, \hat{X}_{2}\right]=0
$$

A completely analogous argument to that given at the end of the previous subsection shows that the linear sections of the form $T(X), X \in \Gamma(A)$ (example 5.1.11), and the core sections span the set of sections $\Gamma(T A, T M)$, therefore the bracket in equations (5.9) is well-defined. The anchor $\rho_{T}$ is defined by $\rho_{T}=J^{-1} \circ T(\rho)$ where

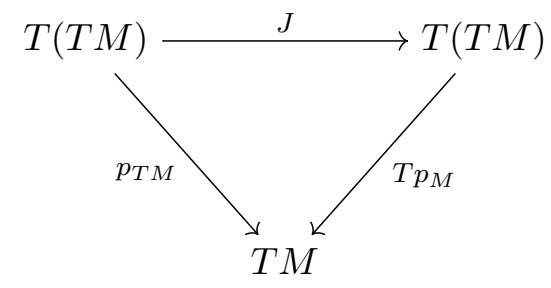

is the involution map (isomorphism) of the double tangent bundle $T(T M)$, determined locally by $J\left(x_{i}, \dot{x}_{i}, \delta x_{i}, \delta \dot{x}_{i}\right)=\left(x_{i}, \delta x_{i}, \dot{x}_{i}, \delta \dot{x}_{i}\right)$, where for local coordinates $\left(x^{i}\right)$ of $M,\left(\dot{x}^{i}\right)$ are the coordinates on the fibers of $T M$ and $\left(\delta x^{i}, \delta \dot{x}^{i}\right)$ are the coordinates on the fibers of $T(T M) \stackrel{p_{T M}}{\longrightarrow}$ $T M$.

Example 5.1.14 (Cotangent Lie algebroid) It is well known that a Lie algebroid $A \longrightarrow M$ induces a Poisson structure on its dual $A^{*} \longrightarrow M$ which is linear with respect to the vector bundle structure over $M$ (Courant (1990), Thm. 2.1.4). In this way, the cotangent bundle of A, $R_{A}^{-1}$

$T^{*} A \stackrel{{ }_{A}}{\cong} T^{*} A^{*} \longrightarrow A^{*}$ inherits a Lie algebroid structure which is further a VB-algebroid when considered over $A \longrightarrow M$. This structure of VB-algebroid also can be described in a simple way by using the natural notion of dual VB-algebroid explained in (Gracia-Saz and Mehta (2010a), Thm 3.1).

The two previous examples have another interesting property: they are (isomorphic to) the associated Lie algebroids of the tangent and cotangent groupoids (section 4.1), respectively. In fact, the isomorphism $J_{\mathcal{G}}: T(T \mathcal{G}) \longrightarrow T(T \mathcal{G})$, given by the involution map, restricts to the isomorphism of Lie algebroids

$$
T A_{\mathcal{G}} \stackrel{j_{\mathcal{G}}}{\longrightarrow} A_{T \mathcal{G}}
$$


over TM (Mackenzie (2005a), Thm 9.7.5). For the isomorphism of the cotangent algebroid, one considers the dual of $J_{\mathcal{G}}$. Explicitely, the isomorphism

$$
\Theta_{T \mathcal{G}}:=J_{\mathcal{G}}^{*} \circ I_{T \mathcal{G}}: T\left(T^{*} \mathcal{G}\right) \longrightarrow(T(T \mathcal{G}))_{T M}^{*} \longrightarrow T^{*}(T \mathcal{G})
$$

given by the composition of the internalization map and the dual of the involution induces the isomorphism (Mackenzie (2005a), p. 463)

$$
A_{T^{*} \mathcal{G}} \stackrel{\theta_{\mathcal{G}}}{\longrightarrow} T^{*} A_{\mathcal{G}}
$$

The map $\Theta_{T \mathcal{G}}$ is often called the Tulczyjew map, and has another interesting property when related with the canonical symplectic structure $\omega_{\text {can }}$ on the cotangent bundle $T^{*} \mathcal{G}$.

Proposition 5.1.15 (Mackenzie (2005a), Thm 9.6.7) Given a manifold M, the Tulczyjew and reversal isomorphisms, $\Theta_{T M}$ and $R_{T M}$, are related by the following commutative diagram

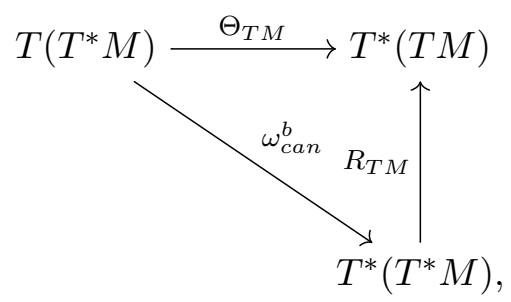

where $\omega_{\text {can }} \in \Omega^{2}\left(T^{*} M\right)$ is the canonical symplectic structure on the cotangent bundle of $M$.

\subsubsection{VB-algebroid complex}

VB-algebroids also have their own cohomology $C_{V B}^{*}(D)$ induced from the algebroid cohomology (of $D \longrightarrow E$ ) when considered its linear structure over $A$. In fact, for a VB-algebroid $D \longrightarrow E$, the VB-algebroid complex is defined as the subcomplex of linear cochains of $C^{*}(D)$ (the algebroid cohomology of $D$ ); i.e., $C_{V B}^{*}(D):=C_{l i n}^{*}(D) \subset C^{*}(D)$ in the following sense: regard the elements of $C^{k}(D)=\Gamma\left(E, \Lambda^{k} D_{E}^{*}\right)$ as the $k$-multilinear and alternating functions $\oplus_{E}^{k} D \longrightarrow \mathbb{R}$; the linear $k$-cochains of $D$ are the elements in $C^{k}(D)$ which are fiberwise linear with respect to the vector bundle structure of $\oplus_{E} D$ over $\oplus_{M} A$. The space $C_{V B}^{*}(D)$ defines in fact a complex, it was shown by Cabrera and Drummond in Cabrera and Drummond (2017), where the authors defined this complex.

\subsection{Deformation cohomology of Lie algebroids}

The deformation complex of Lie algebroids was defined by Crainic and Moerdijk in (Crainic and Moerdijk, 2004) when working in the deformation theory of Lie algebroids, and it is one more example emphasizing the general "principle" of the deformation theory. For a 
Lie algebroid $A$, this complex is denoted by $\left(C_{d e f}^{*}(A), \delta\right)$. For its definition first we need the notion of multiderivations on a vector bundle.

Let $E \longrightarrow M$ be a vector bundle. A derivation on $E$ is a linear operator $D: \Gamma(E) \longrightarrow \Gamma(E)$ such that there exists a vector field $\sigma_{D} \in \mathfrak{X}(M)$, called the symbol of $D$ which satisfies

$$
D(f s)=f D(s)+\sigma_{D}(f) s, \text { for } s \in \Gamma(E) \text { and } f \in C^{\infty}(M) .
$$

A multiderivation of degree $n$ on $E$ is a multilinear and antisymmetric map

$$
D: \underbrace{\Gamma(E) \times \cdots \times \Gamma(E)}_{n+1 \text { times }} \longrightarrow \Gamma(E)
$$

which is a derivation in each entry, i.e., there is a symbol map

$$
\sigma_{D}: \underbrace{\Gamma(E) \times \cdots \times \Gamma(E)}_{n \text { times }} \longrightarrow \mathfrak{X}(M)
$$

which is $C^{\infty}(M)$-linear in each entry and satisfies

$$
D\left(s_{0}, s_{1}, \ldots, f s_{n}\right)=f D\left(s_{0}, \ldots, s_{n}\right)+\sigma_{D}\left(s_{0}, \ldots, s_{n-1}\right)(f) s_{n}, \text { for } s_{i} \in \Gamma(E) \text { and } f \in C^{\infty}(M)
$$

The set of multiderivations of degree $n$ is often denoted by $\operatorname{Der}^{n}(E)$. One sets $\operatorname{Der}^{-1}(E)=$ $\Gamma(E)$.

In this way, the deformation complex of $A$ is the complex in which the set of $k$-cochains $C_{d e f}^{k}(A)$ consists of the multiderivations of degree $k-1$ on $A$, i.e., $C_{d e f}^{k}(A)=\operatorname{Der}^{k-1}(A)$, with differential given by

$$
\begin{aligned}
\delta(D)\left(\alpha_{0}, \ldots, \alpha_{k}\right)=\Sigma_{i}(-1)^{i}\left[\alpha_{i},\right. & \left.D\left(\alpha_{0}, \ldots, \hat{\alpha}_{i}, \ldots, \alpha_{k}\right)\right]+ \\
& +\Sigma_{i<j}(-1)^{i+j} D\left(\left[\alpha_{i}, \alpha_{j}\right], \alpha_{0}, \ldots, \hat{\alpha}_{i}, \ldots, \hat{\alpha}_{j}, \ldots, \alpha_{k}\right) .
\end{aligned}
$$

We now revisit the map $i$ introduced in Crainic and Moerdijk (2004). Such a map is defined in terms of cochains by $i: C_{\pi}^{k}(M) \rightarrow C_{d e f}^{k}\left(T^{*} M\right), X \mapsto D_{X}$, where $D_{X} \in \operatorname{Der}^{k-1}\left(T^{*} M\right)$ acts on exact forms by

$$
D_{X}\left(d f_{1}, \ldots, d f_{k}\right):=d\left(X\left(f_{1}, \ldots, f_{k}\right)\right)
$$

A general expression for the multiderivation $D_{X}$ can also be obtained (see Crainic and Moerdijk (2004), Prop. 3), but the previous formula is enough for our purporses. In order to can study the map $i$ in another way, we use the notion of the tangent lift of $k$-multivector fields, $T: \mathfrak{X}^{k}(M) \longrightarrow \mathfrak{X}_{\text {lin }}^{k}(T M) \subset \mathfrak{X}^{k}(T M)$, defined as follows. Regard the $k$-vector fields as the $k$-multilinear functions 


$$
\mathfrak{X}^{k}(M)=\Gamma\left(\bigwedge_{p_{M}}^{k} T M\right)=\left\{\bigoplus_{c_{M}}^{k} T^{*} M \stackrel{k \text {-multilinear }}{\longrightarrow} \mathbb{R}\right\}
$$

Thus,

$$
\mathfrak{X}^{k}(T M)=\Gamma\left(\bigwedge_{p_{T M}}^{k} T(T M)\right)=\left\{\bigoplus_{c_{T M}}^{k} T^{*}(T M) \stackrel{k \text {-multilinear }}{\longrightarrow} \mathbb{R}\right\} .
$$

Then, if $X \in \mathfrak{X}^{k}(M)$, its tangent lift is given by

$$
\tilde{X}:=T X \circ \oplus^{k} \Theta_{T M}^{-1}: \bigoplus_{c_{T M}}^{k} T^{*}(T M) \longrightarrow \bigoplus_{T c_{M}}^{k} T\left(T^{*} M\right) \longrightarrow \mathbb{R} .
$$

Note further that, in this way, $\tilde{X}$ is (fiberwise) linear with respect to the vector bundle structure of $\bigoplus_{c_{T M}}^{k} T^{*}(T M)$ over $\bigoplus_{c_{M}}^{k} T^{*} M$ (TX is (fiberwise) linear with respect to the bundle projection $\oplus^{k} p_{T^{*} M}$ and $\oplus \Theta_{T M}$ is an isomorphism of DVBs). That is $\tilde{X} \in \mathfrak{X}_{\text {lin }}^{k}(T M)$, the tangent lift of the multivector field $X \in \mathfrak{X}^{k}(M)$ is a linear multivector on $T M$.

Now we remark that, by using the tangent lift of multivector fields, $T$, we can see the map $i$ as the composition

$$
\mathfrak{X}^{k}(M) \stackrel{T}{\longrightarrow} \mathfrak{X}_{\text {lin }}^{k}(T M) \stackrel{D_{T M}}{\longrightarrow} \operatorname{Der}^{k-1}\left(T^{*} M\right)
$$

where $D_{T M}$ is a particular case of the map (isomorphism) $D_{E}: \mathfrak{X}_{l i n}^{k}(E) \longrightarrow \operatorname{Der}^{k-1}\left(E^{*}\right)$ defined in (Crainic and Moerdijk (2004), section 4.9) for any vector bundle $E$ over $M$ by $D_{E}(X)\left(s_{1}, \ldots, s_{k}\right):=X\left(l_{s_{1}}, \ldots, l_{s_{k}}\right)$, with $s_{i} \in \Gamma\left(E^{*}\right)$ and $l: \Gamma\left(E^{*}\right) \stackrel{\cong}{\longrightarrow} C_{\text {lin }}^{\infty}(E)$ the correspondence which views a section $s \in \Gamma\left(E^{*}\right)$ as a (fiberwise) linear function on $E$.

In fact (by simplicity we verify for $k=2$ ), denoting (also) by $l: \mathfrak{X}^{2}(M) \longrightarrow C_{2-l i n}^{\infty}\left(\bigoplus_{c_{M}}^{2} T^{*} M\right.$ ) the function which regard the bivector fields on a manifold $M$ as bilinear and antisymmetric functions on $\bigoplus_{c_{M}}^{2} T^{*} M$ we get

$$
\begin{aligned}
D_{T M}(\tilde{X})\left(d f_{1}, d f_{2}\right) & =\tilde{X}\left(l_{d f_{1}}, l_{d f_{2}}\right) \\
& =l_{\tilde{X}}\left(d \tilde{f}_{1}, d \tilde{f}_{2}\right) \quad\left(\text { where } \tilde{f}_{i}:=T f_{i} \equiv l_{d f_{i}} \text { is the tangent lift of } f_{i}\right) \\
& =T\left(l_{X}\right) \circ \Theta_{T M}^{-1}\left(d \tilde{f}_{1}, d \tilde{f}_{2}\right) \\
& =T\left(l_{X}\right)\left(T\left(d f_{1}\right), T\left(d f_{2}\right)\right) \\
& =T\left(l_{X}\left(d f_{1}, d f_{2}\right)\right) \\
& =d\left(l_{X}\left(d f_{1}, d f_{2}\right)\right) \\
& =d\left(X\left(f_{1}, f_{2}\right)\right) \quad(\text { by definition of } l) \\
& =i(X)\left(d f_{1}, d f_{2}\right) .
\end{aligned}
$$


where in the fourth equality we use the fact that $\Theta_{T M} \circ T(d f)=d \tilde{f}$, see Mackenzie (2005a) p. 394.

Remark 5.2.1 Note that, as pointed out in (Crainic and Moerdijk (2004), Prop. 7), the map $D_{E}$ defined above, when $E=A^{*}$ (the dual of the Lie algebroid $A$ ), provides us with an interpretation of the deformation complex of Lie algebroids in terms of the VB-algebroid complex of the VB-algebroid $T^{*} A^{*} \longrightarrow A^{*}$ viewed over the algebroid $A \longrightarrow M$. In fact, $\mathfrak{X}_{\text {lin }}^{k}\left(A^{*}\right)=C_{V B}^{k}\left(T^{*} A^{*}\right)$, and $D_{A^{*}}: \mathfrak{X}_{\text {lin }}^{\bullet}\left(A^{*}\right) \longrightarrow C_{\text {def }}^{\bullet}(A)$ turns out to be an isomorphism of differential graded Lie algebras, where on the left one considers the Schouten bracket of multivector fields.

Remark 5.2.2 In the context developed in this subsection (M Poisson manifold), the Tulczyjew map $\Theta_{T M}: T\left(T^{*} M\right) \longrightarrow T^{*}(T M)$ has an additional interesting property: it is an isomorphism of VB-algebroids, between the tangent algebroid and the Lie algebroid associated to the linear Poisson structure on TM (Mackenzie (2005a), Prop. 10.3.13).

\section{$5.3 i_{\mathcal{G}}$ and van-Est commutativity}

Consider $\left\{\left(\mathcal{G}_{\epsilon}, \omega_{\epsilon}\right)\right\}_{\epsilon}$ an $s$-constant deformation of $(\mathcal{G}, \omega)$, with $(M, \pi)$ the Poisson structure induced on the units of $\mathcal{G}$. By applying the Lie functor to $\left\{\left(\mathcal{G}_{\epsilon}, \omega_{\epsilon}\right)\right\}_{\epsilon}$ one looks at two types of deformations: one deformation of Poisson structures on $M$, and another one of Lie algebroids $A_{\mathcal{G}_{\epsilon}}$. Of course, such deformations are not independent of each other, the deformation of Lie algebroids is isomorphic to the one induced from the deformation of Poisson structures (when looking at the Lie algebroids underlying to such Poisson structures). More generally, every deformation of $\pi$ can be viewed as a deformation of $A_{\mathcal{G}}$ as a Lie algebroid, but not conversely. This latter fact has an analogue in terms of infinitesimal deformations given by the definition of the map $i: H_{\pi}^{*}(M) \longrightarrow H_{\text {def }}^{*}\left(A_{\mathcal{G}}=\left(T^{*} M\right)_{\pi}\right)$ Crainic and Moerdijk (2004). In this sense, we now define a map $i_{\mathcal{G}}: H_{\text {diff }}^{*}(\mathcal{G}) \longrightarrow H_{\text {def }}^{*}(\mathcal{G})$ which will be the global analogue of such a map $i$ (for integrable Poisson structures). That is, we describe the map $i_{\mathcal{G}}$ in the case of $\mathcal{G}$ being a symplectic groupoid.

Recall from Remark 4.1.6 that $H_{d e f}^{\bullet}(\mathcal{G}) \cong H_{V B}^{\bullet}\left(T^{*} \mathcal{G}\right)$, and moreover, by Lemma 3.1 in Cabrera and Drummond (2017), $H_{V B}^{\bullet}\left(T^{*} \mathcal{G}\right) \cong H_{\text {lin }}^{\bullet}\left(T^{*} \mathcal{G}\right)$, therefore defining a map (between cohomologies!) $H^{\bullet}(\mathcal{G}) \longrightarrow H_{\text {def }}^{\bullet}(\mathcal{G})$ amounts to defining another map $j: H^{\bullet}(\mathcal{G}) \longrightarrow$ $H_{\text {lin }}^{\bullet}\left(T^{*} \mathcal{G}\right)$. The latter is defined in terms of cochains by

$$
C^{k}(\mathcal{G}) \stackrel{T}{\longrightarrow} C_{l i n}^{k}(T \mathcal{G}) \stackrel{\left(\omega^{\#}\right)^{*}}{\longrightarrow} C_{\text {lin }}^{k}\left(T^{*} \mathcal{G}\right)
$$

where $\omega^{\#}:=\left(\omega^{b}\right)^{-1}: T^{*} \mathcal{G} \rightarrow T \mathcal{G}$ and $T$, the natural tangent lift of groupoid-cochains, we define by using the canonical identification $(T \mathcal{G})^{(k)} \cong T \mathcal{G}^{(k)} ; T(c):\left(v_{g_{1}}, \ldots, v_{g_{k}}\right) \mapsto$ $d c\left(v_{g_{1}}, \ldots, v_{g_{k}}\right)$. 
Notorious of this map $i_{\mathcal{G}}$ is that if $\mathcal{G}$ is $s$-connected and $(M, \pi)$ is the Poisson structure induced on the base (i.e. the infinitesimal information associated to $(\mathcal{G}, \omega)$ ), we can look at the map $i: H_{\pi}(M) \longrightarrow H_{d e f}\left(T^{*} M\right)$ as the infinitesimal data corresponding to $i_{\mathcal{G}}$ in the sense that we have the following commutative diagram,

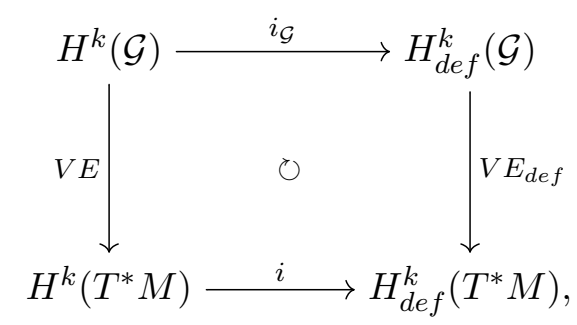

where $V E_{\text {def }}$ is the van-Est map between the deformation cohomologies as defined in Cabrera and Drummond (2017). That is,

Theorem 5.3.1 Let $(\mathcal{G}, \omega)$ be a symplectic groupoid. If $\mathcal{G}$ is s-connected, the map $i_{\mathcal{G}}: H^{*}(\mathcal{G}) \stackrel{j}{\rightarrow}$ $H_{\text {lin }}^{*}\left(T^{*} \mathcal{G}\right) \cong H_{\text {def }}^{*}(\mathcal{G})$, as defined above, is the global counterpart of the map $i$. That is, $i_{\mathcal{G}}$ together with $i$ and the respective van-Est maps (of the differentiable and deformation cohomology) form the commutative diagram (5.13).

The proof of this theorem is based on some lemmas. The first one refers to the tangent lift of algebroid cochains, analogue to the tangent lift of groupoid cochains described above.

Lemma 5.3.2 Let $A$ be a Lie algebroid over $M$. The tangent lift determines a cochain map

$$
T: C^{k}(A) \longrightarrow C_{l i n}^{k}(T A) \subset C^{k}(T A),
$$

between algebroid complexes. We refer to this map as the tangent lift of algebroid cochains.

\section{Proof of Theorem 5.3.1}

The proof follows from working on each of the properties of the symplectic form $\omega$ (nondegeneracy, multiplicativity and closedness). First note that, from non-degeneracy and mul- 
tiplicativity, the map $j$ fits into the following diagram

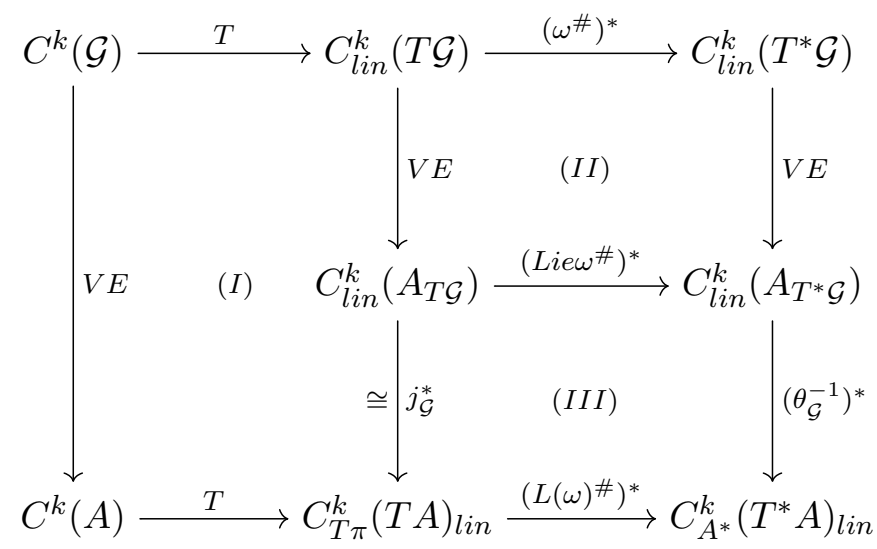

Indeed, diagram $(I)$ (to be proved in subsection 5.3 .1 below) is a general fact relating the tangent lifts of cochains on Lie groupoids and Lie algebroids by the van-Est map. Diagram $(I I)$ follows from the naturality of the van-Est map with respect to morphisms of Lie groupoids (Lemma 2.10 Cabrera and Drummond (2017)). And diagram (III) amounts to regarding the morphism of Lie algebroids $\operatorname{Lie}\left(\omega^{b}\right): A_{T \mathcal{G}} \rightarrow A_{T^{*} \mathcal{G}}$ in terms of the (canonically) isomorphic Lie algebroids $T A_{\mathcal{G}}$ and $T^{*} A_{\mathcal{G}}$; denote it by $L(\omega)^{b}: T A_{\mathcal{G}} \longrightarrow T^{*} A_{\mathcal{G}}$. Further, because this latter morphism is the infinitesimal counterpart of a morphism of Lie groupoids induced by a 2 -form on $\mathcal{G}$, it also turns out to be induced by a 2 -form $L(\omega)$ on $A$ (which makes sense to the notation $L(\omega)^{b}$ above). In fact, one can describe explicitely such a 2 -form $L(\omega)$ by using the tangent lift, $\omega^{T} \in \Omega^{2}(T \mathcal{G})$, of the form $\omega \in \Omega^{2}(\mathcal{G})$ getting $L(\omega)=\iota_{A}^{*} \omega^{T}$, where $\iota_{A}$ is the inclusion map $A_{\mathcal{G}} \hookrightarrow T \mathcal{G}$; this point is developed in (Bursztyn et al. (2009), Prop. 3.7).

So, now by putting together the reversal isomorphism $T^{*} A^{*} \stackrel{R_{A}}{\longrightarrow} T^{*} A$ and the map (isomorphism) $D_{A^{*}}$ defined above (section 5.2), with the lower part of diagram (5.14), we get

$$
\cdots C_{T \pi}^{k}(T A)_{l i n} \stackrel{\left(L(\omega)^{\#}\right)^{*}}{\longrightarrow} C_{A^{*}}^{k}\left(T^{*} A\right)_{l i n} \stackrel{R_{A}^{*}}{\longrightarrow} C_{A^{*}}^{k}\left(T^{*} A^{*}\right)_{l i n} \stackrel{D_{A^{*}}}{\longrightarrow} \operatorname{Der}^{k-1}(A) .
$$

Then, by diagram (5.14) and sequence (5.15), we have the following commutative diagram 
in cohomology

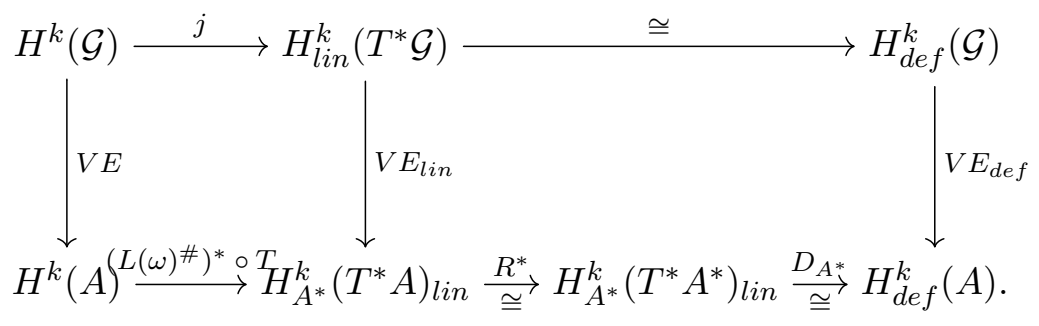

On the other hand, by considering the fact that $\omega$ is also closed, we get both on $M$ a Poisson structure $\pi$ and, infinitesimally, an isomorphism of Lie algebroids $\sigma: A_{\mathcal{G}} \rightarrow\left(T^{*} M\right)_{\pi}$. Therefore, by using the expression (5.12) of the map $i$, we have the following commutative diagrams.

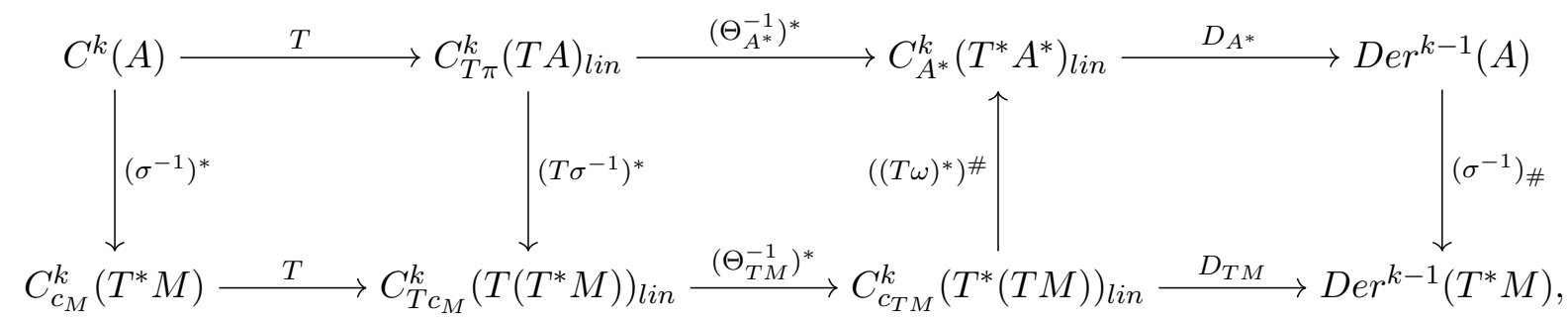

where $\Theta_{A^{*}}$ is the isomorphism of VB-algebroids (isomorphism of DVBs preserving the Lie algebroid structures involved) induced from $\Theta_{T M}$ by using the isomorphism $\sigma: A \longrightarrow T^{*} M$. That is, $\Theta_{A^{*}}=\left(\left(T \sigma^{*}\right)_{\left(\sigma^{*}\right)^{-1}}^{\star}\right)^{-1} \circ \Theta_{T M} \circ T \sigma: T A \longrightarrow T^{*} A^{*}$ (remark 5.1.7), which for simplicity we just write $\left(\left(T \sigma^{*}\right)^{*}\right)^{-1} \circ \Theta_{T M} \circ T \sigma$.

Then, by comparing the upper part of diagram above with the previous sequence of maps (5.15), we have two isomorphisms of VB-algebroids: $L(\omega)^{\#} \circ R_{A}$ and $\Theta_{A^{*}}^{-1}$ from $T^{*} A^{*}$ to $T A$. We show now that these two maps are the same, which completes the proof (just put together diagrams (5.16) and (5.17) in cohomology). In fact,

$$
\begin{aligned}
\Theta_{A^{*}} & =\left(\left(T \sigma^{*}\right)^{*}\right)^{-1} \circ \Theta_{T M} \circ(T \sigma) \\
& =\left(\left(T \sigma^{*}\right)^{*}\right)^{-1} \circ\left(R_{T M} \circ \omega_{c a n}^{b}\right) \circ T \sigma \quad(\text { Prop. 5.1.15) } \\
& =R_{A^{*}} \circ(T \sigma)^{*} \circ \omega_{c a n}^{b} \circ T \sigma \quad \text { (Prop. 5.1.9) } \\
& =R_{A}^{-1} \circ\left(\sigma^{*} \omega_{c a n}\right)^{b} \\
& =R_{A}^{-1} \circ L(\omega)^{b} ;
\end{aligned}
$$

where $R_{A^{*}}=R_{A}^{-1}$ is easily verified from a local point of view, and the last step follows from the characterization of the linear 2 -forms on $A_{\mathcal{G}}$ coming from multiplicative 2 -forms on $\mathcal{G}$ 
(Bursztyn et al. (2009), Prop. 4.6).

Remark 5.3.3 Note that, in view of (Cabrera and Drummond (2017), Thm. 2.14), the map $V E_{\text {def }}$ considered above, is a map which, under the same $k$-connectedness condition on the $s$ fibers of $\mathcal{G}$, satisfies the same properties of the van-Est map $\widetilde{V E}_{\text {def }}$ as defined in (Crainic et al. (2015a), Thm 10.1); and due to that one calls VE def also a "van-Est type map" between the deformation cohomologies. In this sense, it would be interesting to understand the underlying relation between such "two van-Est maps".

Remark 5.3.4 (IM-2-forms)

It is worth to say that besides considering the map $\sigma$ for $\omega$ closed (and multiplicative), this map also makes sense even if $\omega$ is only multiplicative (not necessarily closed); in that case $\sigma: A_{\mathcal{G}} \longrightarrow T^{*} M$ is a vector-bundle map which satisfies some relations involving the Lie bracket on sections of $A$ and the Lie derivative, interior product and exterior differential on sections of $T^{*} M$. In the literature, such a map $\sigma$ is called the IM-2-form associated to the multiplicative 2-form $\omega$. Further, an abstract definition of IM-2-forms also can be considered [Bursztyn et al. (2004), Bursztyn et al. (2009), Bursztyn and Cabrera (2012), Crainic et al. (2015b)]. Analogously, multiplicative $k$-forms on $\mathcal{G}$ lead to the notion of IM-k-forms (see e.g. Bursztyn and Cabrera (2012)).

\subsubsection{Proof of diagram $(I)$}

The goal of this section is to complete the proof of theorem 5.3.1 by proving the commutativity of diagram $(I)$ in (5.14) above. Such a diagram involves the tangent Lie structures (tangent groupoid and algebroid) and the tangent lift of cochains. In this way, the arguments exposed here will be based on the two kinds of special sections of the tangent algebroid: core sections and tangent lift of sections (example 5.1.13). They are the fundamental sections of this algebroid in the sense that they span (as a $C^{\infty}(M)$-module) all the other sections of it, which allows to simplify the proofs below.

Lemma 5.3.5 Consider $k$ a positive integer. The tangent lift of Lie algebroid cochains $T$ : $C^{k}(A) \longrightarrow C^{k}(T A)$ (lemma 5.3.2) satisfies the following conditions:

1. (Linear sections) $\left.T c\left(T \alpha_{1}, \ldots, T \alpha_{k}\right)\right|_{w_{x}}=(T c)_{w_{x}}\left(T \alpha_{1}\left(w_{x}\right), \ldots, T \alpha_{k}\left(w_{x}\right)\right)=\left[T\left(c\left(\alpha_{1}, \ldots, \alpha_{k}\right)\right)\right]\left(w_{x}\right)$,

2. (One core-section) $\left.T c\left(T \alpha_{1}, \ldots, \hat{\alpha}_{k}\right)\right|_{w_{x}}=\left.c\left(\alpha_{1}, \ldots, \alpha_{k}\right)\right|_{x}$,

3. (More than one core-sections) $\left.T c\left(T \alpha_{1}, \ldots, \hat{\alpha}_{k-1}, \hat{\alpha}_{k}\right)\right|_{w_{x}}=0$.

\section{Proof.}

1. The first statement is direct. For simplicity, we prove the following properties for $k=2$, the general case $(k \neq 2)$ is totally analogous. 
2.

$$
\begin{aligned}
\left.T c\left(T \alpha_{1}, \hat{\alpha}_{2}\right)\right|_{w_{x}} & =(T c)_{\left(w_{x}\right)}\left(T \alpha_{1}\left(w_{x}\right), \hat{\alpha}_{2}\left(w_{x}\right)\right) \\
& =(T c)_{\left(w_{x}\right)}(\overbrace{T \alpha_{1}\left(w_{x}\right)}^{\in T_{\alpha_{1}(x)} A}, T 0^{A}\left(w_{x}\right)+{ }_{A}\left(\alpha_{2}^{\uparrow}\right)_{0_{x}^{A}}) \\
& =\underbrace{(T c)_{\left(w_{x}\right)}\left(T \alpha_{1}\left(w_{x}\right), T 0^{A}\left(w_{x}\right)\right)}_{=0}+ \\
& +(T c)_{\left(0_{x}^{A}\right)}\left(\tilde{0}_{\alpha_{1}(x)}^{A},\left(\alpha_{2}^{\uparrow}\right)_{0_{x}^{A}}\right) \quad\left(\text { Linearity of } T c \text { over } \oplus_{M}^{2} A\right) \\
& =\left.\frac{d}{d \lambda}\right|_{\lambda=0} c\left(\alpha_{1}(x), \lambda \alpha_{2}(x)\right) \\
& =\left.c\left(\alpha_{1}, \alpha_{2}\right)\right|_{(x)} \quad(\text { Bilinearity of } c(\text { over } M)),
\end{aligned}
$$

where in the third equality the first term vanishes by the multilinearity of $T c$ with respect to the vector bundle $T A \longrightarrow T M$.

3. Finally, considering more than one core-section:

$$
\begin{aligned}
\left.T c\left(\hat{\alpha}_{1}, \hat{\alpha}_{2}\right)\right|_{w_{x}} & =T c\left(T 0^{A}\left(w_{x}\right)+_{A}\left(\alpha_{1}^{\uparrow}\right)_{0_{x}^{A}}, T 0^{A}\left(w_{x}\right)+_{A}\left(\alpha_{2}^{\uparrow}\right)_{0_{x}^{A}}\right) \\
& =T c\left(T 0^{A}\left(w_{x}\right), T 0^{A}\left(w_{x}\right)\right)+T c\left(\left(\alpha_{1}^{\uparrow}\right)_{0_{x}^{A}},\left(\alpha_{2}^{\uparrow}\right)_{0_{x}^{A}}\right) \\
& =0+\left.\frac{d}{d \lambda}\right|_{\lambda=0} c\left(\lambda \alpha_{1}(x), \lambda \alpha_{2}(x)\right) \\
& =\left.\frac{d}{d \lambda}\right|_{\lambda=0} \lambda^{2} \cdot c\left(\alpha_{1}(x), \alpha_{2}(x)\right) \\
& =0
\end{aligned}
$$

where in the third equality $T c\left(T 0^{A}\left(w_{x}\right), T 0^{A}\left(w_{x}\right)\right)=0$ by the multilinearity of $T c$ with respect to the vector bundle $T A \longrightarrow T M$.

Note that the key fact in order to extend these proofs to the general case $k \neq 2$ is using the linearity of $T c$ to get a sum of zero plus a more simple expression in terms of $c$ and sections of $A_{\mathcal{G}}$.

$\diamond$

We can now pass to prove the commutativity of $(I)$. For that, recall the definition of the van-Est map Crainic (2003), $V E: C^{k}(\mathcal{G}) \longrightarrow C^{k}\left(A_{\mathcal{G}}\right)$, between the differentiable cohomology of a Lie groupoid and the algebroid cohomology of its associated Lie algebroid. Given $c \in$ $C^{k}(\mathcal{G}), V E(c)$ is defined by

$$
V E(c)\left(X_{1}, \ldots, X_{k}\right)=\sum_{\sigma \in S_{n}} \operatorname{sgn}(\sigma) R_{\sigma\left(X_{1}\right)} \circ \cdots \circ R_{\sigma\left(X_{k}\right)} c ; \text { for } X_{i} \in \Gamma\left(A_{\mathcal{G}}\right),
$$


where if $X \in \Gamma\left(A_{\mathcal{G}}\right), R_{X}$ is the map $R_{X}: C^{k}(\mathcal{G}) \longrightarrow C^{k-1}(\mathcal{G})$ given by

$$
R_{X} c\left(g_{1}, \ldots, g_{k-1}\right)=\left.\frac{d}{d \epsilon}\right|_{\epsilon=0} c\left(\psi_{\epsilon}^{\vec{X}}\left(t\left(g_{1}\right)\right), g_{1}, \ldots, g_{k}-1\right)
$$

with $\psi_{\epsilon}^{\vec{X}}$ being the flow of the right invariant vector field associated to $X \in \Gamma\left(A_{\mathcal{G}}\right)$.

In this way note that, if $c \in C^{2}(\mathcal{G})$, by definition of $R_{X}$ we have

$$
\left(R_{X_{1}} R_{X_{2}} c\right)_{(x)}=\left.\left.\frac{d}{d \epsilon_{1}}\right|_{\epsilon_{1}=0} \frac{d}{d \epsilon_{2}}\right|_{\epsilon_{2}=0} c\left(\psi_{\epsilon_{2}}^{\vec{X}_{2}}\left(\phi_{\epsilon_{1}}^{V_{1}}(x)\right), \psi_{\epsilon_{1}}^{\vec{X}_{1}}(x)\right),
$$

where $V_{1} \in \mathfrak{X}(M)$ is the vector field on $M, \rho\left(X_{1}\right)$ (the projection by the anchor).

In general, for $c \in C^{k}(\mathcal{G})$ and $X_{1}, \ldots, X_{k}$ sections of $A_{\mathcal{G}}$,

$\left(R_{X_{1}} \cdots R_{X_{k}} c\right)_{(x)}=\left.\left.\frac{d}{d \epsilon_{1}}\right|_{0} \cdots \frac{d}{d \epsilon_{k}}\right|_{0} c\left(\psi_{\epsilon_{k}}^{\vec{X}_{k}}\left(\psi_{\epsilon_{k-1}}^{V_{k-1}} \cdots \psi_{\epsilon_{1}}^{V_{1}}(x)\right), \psi_{\epsilon_{k-1}}^{\vec{X}_{k-1}}\left(\psi_{\epsilon_{k-2}}^{V_{k-2}} \cdots \psi_{\epsilon_{1}}^{V_{1}}(x)\right), \ldots, \psi_{\epsilon_{1}}^{\vec{X}_{1}}(x)\right)$.

Therefore, all the essence is captured by considering the case $k=2$, which we will prove explicitly below with all the details, and we will coment the way to apply them for the general case (which yields an analogous proof).

In order to use formula (5.18) for sections of the tangent Lie algebroid, we study the flow of the appropriate right-invariant vector fields on $T \mathcal{G}$. We then, analogous to lemma 5.3.5, split the proof in three cases:

1. Linear sections: $\left(X_{i}=j_{\mathcal{G}} \circ\left(T \alpha_{i}\right), \alpha_{i} \in \Gamma\left(A_{\mathcal{G}}\right), i=1,2.\right)$

In this case, the flow of $\vec{X}_{i} \in \mathfrak{X}(T \mathcal{G})$ is the tangent lift of the flow of $\vec{\alpha}_{i}$ (Mackenzie et al. (1994), Thm 7.1), thus for instance,

$$
\begin{aligned}
\psi_{\epsilon}^{\vec{X}_{i}}\left(T u\left(w_{y}\right)\right) & =\left(T \psi_{\epsilon}^{\vec{\alpha}_{i}}\right)\left(T u\left(w_{y}\right)\right) \\
& =T\left(\psi_{\epsilon}^{\vec{\alpha}_{i}} \circ u\right)\left(w_{y}\right) \\
& =\left.\frac{d}{d \lambda}\right|_{\lambda=0} \psi_{\epsilon}^{\vec{\alpha}_{i}} \circ u(\gamma(\lambda)),
\end{aligned}
$$

for $\gamma(\lambda)$ a curve determining $w_{y}$. 
Then,

$$
\begin{aligned}
\left(R_{X_{1}} R_{X_{2}}(T c)\right)_{\left(w_{x}\right)} & =\left.\left.\frac{d}{d \epsilon_{1}}\right|_{\epsilon_{1}=0} \frac{d}{d \epsilon_{2}}\right|_{\epsilon_{2}=0} d c\left(\left.\frac{d}{d \lambda}\right|_{\lambda=0}\left(\psi_{\epsilon_{2}}^{\vec{\alpha}_{2}}\left(\psi_{\epsilon_{1}}^{V_{1}}(\gamma(\lambda))\right)\right), \psi_{\epsilon_{1}}^{\vec{\alpha}_{1}}((\gamma(\lambda)))\right) \\
& =\left.\frac{d}{d \lambda}\right|_{\lambda=0}\left(R_{\alpha_{1}} R_{\alpha_{2}} c\right)_{(\gamma(\lambda))} \\
& =d\left(R_{\alpha_{1}} R_{\alpha_{2}} c\right)_{\left(w_{x}\right)} .
\end{aligned}
$$

Therefore, it follows that

$$
\left.V E(T c)\left(X_{1}, X_{2}\right)\right|_{w_{x}}=\left.d\left(V E(c)\left(\alpha_{1}, \alpha_{2}\right)\right)\right|_{w_{x}} .
$$

Which, by item (1) in lemma 5.3.5, is

$$
\left.j_{\mathcal{G}}^{*}(V E(T c))\left(T \alpha_{1}, T \alpha_{2}\right)\right|_{w_{x}}=\left.T(V E(c))\left(T \alpha_{1}, T \alpha_{2}\right)\right|_{w_{x}} .
$$

The same argument shows that the equality holds for general $k$-cochains $(k>0)$ if we take only linear sections.

2. One core-section case: Let $\hat{\alpha} \in \Gamma\left(T A_{\mathcal{G}}\right)$ be the core-section associated to $\alpha \in \Gamma\left(A_{\mathcal{G}}\right)$, and $X:=j_{\mathcal{G}} \circ \hat{\alpha}$.

In this case, the right-invariant vector field on $T \mathcal{G}$ associated to $X$ is $\vec{X}=(\vec{\alpha})^{\uparrow}$ (Mackenzie et al. (1994), Thm 7.1), therefore its flow is given by $\psi_{\epsilon}^{(\vec{\alpha})^{\uparrow}}\left(v_{g}\right)=v_{g}+\epsilon \vec{\alpha}_{g}$.

Thus, if $X_{1}:=j_{\mathcal{G}} \circ T \alpha_{1}$ and $X_{2}:=j_{\mathcal{G}} \circ \hat{\alpha}_{2}$, denote by $u_{1}=\rho\left(\alpha_{1}\right) \in \mathfrak{X}(M)$ :

$$
\begin{aligned}
\left(R_{X_{1}} R_{X_{2}}(T c)\right)_{\left(w_{x}\right)} & =\left.\left.\frac{d}{d \epsilon_{1}}\right|_{\epsilon_{1}=0} \frac{d}{d \epsilon_{2}}\right|_{\epsilon_{2}=0} T c\left(\psi_{\epsilon_{2}}^{\left(\vec{X}_{2}\right)}\left(t_{T \mathcal{G}}\left(\psi_{\epsilon_{1}}^{\vec{X}_{1}}\left(w_{x}\right)\right)\right), \psi_{\epsilon_{1}}^{\vec{X}_{1}}\left(w_{x}\right)\right) \\
& =\left.\left.\frac{d}{d \epsilon_{1}}\right|_{\epsilon_{1}=0} \frac{d}{d \epsilon_{2}}\right|_{\epsilon_{2}=0} T c(\underbrace{T \psi_{\epsilon_{1}}^{u_{1}}\left(w_{x}\right)}_{w_{\psi_{\epsilon_{1}}^{\prime}(x)}^{u_{1}}}+\left.\epsilon_{2} \vec{\alpha}_{2}\right|_{\psi_{\epsilon_{1}}(x)} ^{u_{1}}, T \psi_{\epsilon_{1}}^{\vec{\alpha}_{1}}\left(w_{x}\right)) \\
& =\left.\frac{d}{d \epsilon_{1}}\right|_{\epsilon_{1}=0} \underbrace{\left.\frac{d}{d \epsilon_{2}}\right|_{\epsilon_{2}=0} T c\left(w_{\varphi_{\epsilon_{1}}^{u_{1}}(x)}^{u_{1}}, T \psi_{\epsilon_{1}}^{\vec{\alpha}_{1}}\left(w_{x}\right)\right)}_{0} \\
& +\left.\left.\frac{d}{d \epsilon_{1}}\right|_{\epsilon_{1}=0} \frac{d}{d \epsilon_{2}}\right|_{\epsilon_{2}=0} d c\left(\left.\epsilon_{2} \vec{\alpha}_{2}\right|_{\varphi_{\epsilon_{1}}^{u_{1}}(x)}, 0_{\psi_{\epsilon_{1}}^{\vec{\alpha}_{1}}(x)}\right) \\
& =\left.\left.\frac{d}{d \epsilon_{1}}\right|_{\epsilon_{1}=0} \frac{d}{d \lambda}\right|_{\lambda=0} c\left(\psi_{\lambda}^{\vec{\alpha}_{2}}\left(\varphi_{\epsilon_{1}}^{u_{1}}(x)\right), \psi_{\epsilon_{1}}^{\vec{\alpha}_{1}}(x)\right) \\
& =\left(R_{\alpha_{1}} R_{\alpha_{2}} c\right)_{(x)},
\end{aligned}
$$


where in the third equality we use the linearity of $T c$ over $\mathcal{G}^{(2)}\left(T c \in C_{\text {lin }}^{2}(T \mathcal{G})\right)$.

Similarly, by changing the order,

$$
\begin{aligned}
\left(R_{X_{2}} R_{X_{1}}(T c)\right)_{\left(w_{x}\right)} & =\left.\left.\frac{d}{d \epsilon_{2}}\right|_{\epsilon_{2}=0} \frac{d}{d \epsilon_{1}}\right|_{\epsilon_{1}=0} T c(\left(T \psi_{\epsilon_{1}}^{\overrightarrow{\alpha_{1}}}\right)(\underbrace{\varphi_{\epsilon_{2}}^{u_{2}^{\uparrow}}\left(w_{x}\right)}_{w_{x}+\epsilon_{2}\left(u_{2}\right)_{x}}), \underbrace{\psi_{\epsilon_{2}}^{\left(\overrightarrow{\alpha_{2}}\right)^{\uparrow}}\left(w_{x}\right)}_{w_{x}+\epsilon_{2}\left(\vec{\alpha}_{2}\right)_{x}}) \\
& =\left.\left.\frac{d}{d \epsilon_{2}}\right|_{\epsilon_{2}=0} \frac{d}{d \epsilon_{1}}\right|_{\epsilon_{1}=0} d c\left(\left(T \psi_{\epsilon_{1}}^{\overrightarrow{\alpha_{1}}}\right)\left(\epsilon_{2}\left(u_{2}\right)_{x}\right), \epsilon_{2}\left(\vec{\alpha}_{2}\right)_{x}\right) \\
& +\left.\left.\frac{d}{d \epsilon_{2}}\right|_{\epsilon_{2}=0} \frac{d}{d \epsilon_{1}}\right|_{\epsilon_{1}=0} d c\left(T \psi_{\epsilon_{1}}^{\overrightarrow{\alpha_{1}}}\left(w_{x}\right), w_{x}\right) \\
& =\left.\left.\frac{d}{d \epsilon_{2}}\right|_{\epsilon_{2}=0} \frac{d}{d \epsilon_{1}}\right|_{\epsilon_{1}=0} \epsilon_{2} d c\left(\left(T \psi_{\epsilon_{1}}^{\overrightarrow{\alpha_{1}}}\right)\left(\left.\frac{d}{d \lambda}\right|_{\lambda=0} \varphi_{\lambda}^{u_{2}}(x)\right),\left.\frac{d}{d \lambda}\right|_{\lambda=0} \psi_{\lambda}^{\vec{\alpha}_{2}}(x)\right) \\
& =\left.\left.\left.\frac{d}{d \epsilon_{2}}\right|_{\epsilon_{2}=0} \frac{d}{d \epsilon_{1}}\right|_{\epsilon_{1}=0} \epsilon_{2} \frac{d}{d \lambda}\right|_{\lambda=0} c\left(\psi_{\epsilon_{1}}^{\overrightarrow{\alpha_{1}}}\left(\varphi_{\lambda}^{u_{2}}(x)\right), \psi_{\lambda}^{\vec{\alpha}_{2}}(x)\right) \\
& =\left.\left.\frac{d}{d \epsilon_{2}}\right|_{\epsilon_{2}=0} \frac{d}{d \epsilon_{1}}\right|_{\epsilon_{1}=0} c\left(\psi_{\epsilon_{1}}^{\overrightarrow{\alpha_{1}}}\left(\varphi_{\epsilon_{2}}^{u_{2}}(x)\right), \psi_{\epsilon_{2}}^{\vec{\alpha}_{2}}(x)\right) \\
& =\left(R_{\alpha_{2}} R_{\alpha_{1}} c\right)(x),
\end{aligned}
$$

where in the second equality we view $d c$ as an element of $C_{\text {lin }}^{2}(T \mathcal{G})$. Hence, it follows that

$$
V E(T c)\left(X_{1}, X_{2}\right)_{\left(w_{x}\right)}=\left(V E(c)_{\left(\alpha_{1}, \alpha_{2}\right)}\right)_{(x)} .
$$

Which, by lemma 5.3 .5 above, is

$$
\left.j_{\mathcal{G}}^{*}(V E(T c))\left(T \alpha_{1}, \hat{\alpha}_{2}\right)\right|_{\left(w_{x}\right)}=\left.T(V E(c))\left(T \alpha_{1}, \hat{\alpha}_{2}\right)\right|_{\left(w_{x}\right)},
$$

as we want.

Note that the main ideas used in the proof of this case are two. $(i)$ in the expression, $\psi_{\epsilon}^{\left(\overrightarrow{\alpha_{2}}\right)^{\uparrow}}\left(v_{x}\right)=v_{x}+\left.\epsilon \vec{\alpha}_{2}\right|_{x}$, for the flow of the right-invariant vector field on $T \mathcal{G}$ associated to the core-section $\hat{\alpha}_{2}$ of $T A_{\mathcal{G}}$, the time parameter $\epsilon$ only is with $\left.\vec{\alpha}_{2}\right|_{x}$, and (ii) by using the linearity of $T c \in C_{\text {lin }}^{2}(T \mathcal{G})$ and $(i)$, we can form two vectors of $(T \mathcal{G})^{(2)}$ : one vector multiplied by $\epsilon_{2}$ and the other one without dependence on $\epsilon_{2}$. Thus, by using these two main facts, a completely analogous argument shows that the equality holds for general $k$-cochains if we have one core-section. In fact, by the skew-symmetry property of the elements of $C^{k}\left(A_{T \mathcal{G}}\right)$, we can assume that $X_{k} \in \Gamma\left(A_{T \mathcal{G}}\right)$ is the core-section in the expression $V E(T c)\left(X_{1}, \ldots, X_{k}\right)$, and in this way the proof of the equality is directly like that above for the case $k=2$.

3. More than one core-section case: $\left(X_{i}:=j_{\mathcal{G}} \circ \hat{\alpha}_{i}, \alpha_{i} \in \Gamma(A), i=1,2.\right)$ 


$$
\begin{aligned}
\left(R_{X_{1}} R_{X_{2}}(T c)\right)_{\left(w_{x}\right)} & =\left.\left.\frac{d}{d \epsilon_{1}}\right|_{\epsilon_{1}=0} \frac{d}{d \epsilon_{2}}\right|_{\epsilon_{2}=0} T c\left(\psi_{\epsilon_{2}}^{\left(\vec{X}_{2}\right)}\left(t_{T \mathcal{G}} \psi_{\epsilon_{1}}^{\vec{X}_{1}}\left(w_{x}\right)\right), \psi_{\epsilon_{1}}^{\left(\vec{X}_{1}\right)}\left(w_{x}\right)\right) \\
& =\left.\left.\frac{d}{d \epsilon_{1}}\right|_{\epsilon_{1}=0} \frac{d}{d \epsilon_{2}}\right|_{\epsilon_{2}=0} T c\left(t_{T \mathcal{G}} \psi_{\epsilon_{1}}^{\left(\vec{\alpha}_{1}\right)^{\uparrow}}\left(w_{x}\right)+\epsilon_{2}\left(\vec{\alpha}_{2}\right)_{x}, w_{x}+\epsilon_{1}\left(\vec{\alpha}_{1}\right)_{x}\right) \\
& =\left.\left.\frac{d}{d \epsilon_{1}}\right|_{\epsilon_{1}=0} \frac{d}{d \epsilon_{2}}\right|_{\epsilon_{2}=0} T c\left(w_{x}+\epsilon_{1}\left(\rho\left(\alpha_{1}\right)\right)_{x}+\epsilon_{2}\left(\vec{\alpha}_{2}\right)_{x}, w_{x}+\epsilon_{1}\left(\vec{\alpha}_{1}\right)_{x}\right) \\
& =\left.\left.\frac{d}{d \epsilon_{1}}\right|_{\epsilon_{1}=0} \frac{d}{d \epsilon_{2}}\right|_{\epsilon_{2}=0} T c\left(w_{x}+\epsilon_{1}\left(\rho\left(\alpha_{1}\right)\right)_{x}, w_{x}+\epsilon_{1}\left(\vec{\alpha}_{1}\right)_{x}\right) \\
& +\left.\left.\frac{d}{d \epsilon_{1}}\right|_{\epsilon_{1}=0} \frac{d}{d \epsilon_{2}}\right|_{\epsilon_{2}=0} T c\left(\epsilon_{2}\left(\vec{\alpha}_{2}\right)_{x}, 0_{x}\right) \\
& =0 .
\end{aligned}
$$

That is, $j_{\mathcal{G}}^{*}[V E(T c)]\left(\hat{\alpha}_{1}, \hat{\alpha}_{2}\right)=0$. Then item (3) in lemma 5.3 .5 completes the commutativity in this case. Therefore, these three cases prove the commutativity of the diagram $(I)$ between tangent lift of cochains (of Lie groupoids and Lie algebroids) and the van-Est maps.

Analogously it is shown that the equality holds for $k$-cochains if we have more than one core-section: it suffices to decompose the vector in $(T \mathcal{G})^{(k)}$ as a sum of two vectors, one depending only on $\epsilon_{1}$ and another depending only on $\epsilon_{2}$. 
74 COHOMOLOGICAL RELATION IN A SYMPLECTIC GROUPOID 


\section{Bibliography}

Bailey and Gualtieri(2016) Michael Bailey and Marco Gualtieri. Integration of generalized complex structures. arXiv preprint arXiv:1611.03850. Cited in p. 1

Bursztyn and Cabrera(2012) Henrique Bursztyn and Alejandro Cabrera. Multiplicative forms at the infinitesimal level. Mathematische Annalen, 353(3):663-705. Cited in p. 68

Bursztyn et al.(2004) Henrique Bursztyn, Marius Crainic, Alan Weinstein, Chenchang Zhu et al. Integration of twisted Dirac brackets. Duke Mathematical Journal, 123(3):549-607. Cited in p. 1, 45, 68

Bursztyn et al.(2009) Henrique Bursztyn, Alejandro Cabrera and Cristián Ortiz. Linear and multiplicative 2-forms. Letters in Mathematical Physics, 90(1-3):59. Cited in p. 43, 66, 68

Bursztyn et al.(2016) Henrique Bursztyn, Alejandro Cabrera and Matias del Hoyo. Vector bundles over lie groupoids and algebroids. Advances in Mathematics, 290:163-207. Cited in p. 39

Cabrera and Drummond(2017) Alejandro Cabrera and Thiago Drummond. Van est isomorphism for homogeneous cochains. Pacific Journal of Mathematics, 287(2):297-336. Cited in p. 61, 64, 65, 66, 68

Coppersmith(1977) Don Coppersmith. Deformations of lie subgroups. Transactions of the American Mathematical Society, 233:355-366. Cited in p. 3, 31, 37

Coste et al.(1987) A Coste, P Dazord and A Weinstein. Groupoïdes symplectiques. Publ. Dept. Math. Lyon, 2(A):1-62. Cited in p. 1, 41

Courant(1990) Theodore James Courant. Dirac manifolds. Transactions of the American Mathematical Society, 319(2):631-661. Cited in p. 60

Crainic(2003) Marius Crainic. Differentiable and algebroid cohomology, van est isomorphisms, and characteristic classes. Commentarii Mathematici Helvetici, 78(4):681-721. Cited in p. 69

Crainic and Fernandes(2011) Marius Crainic and Rui Loja Fernandes. A geometric approach to conn's linearization theorem. Annals of mathematics, pages 1121-1139. Cited in p. 2

Crainic and Moerdijk(2004) Marius Crainic and Ieke Moerdijk. Deformations of lie brackets: cohomological aspects. arXiv preprint math/0403434. Cited in p. iii, v, 4, 5, 51, 61, 62, 63,64 
Crainic and Struchiner(2013) Marius Crainic and Ivan Struchiner. On the linearization theorem for proper lie groupoids. Em Annales scientifiques de l'École Normale Supérieure, volume 46, pages 723-746. Société mathématique de France. Cited in p. 2

Crainic et al.(2014) Marius Crainic, Florian Schätz and Ivan Struchiner. A survey on stability and rigidity results for lie algebras. Indagationes Mathematicae, 25(5):957-976. Cited in p. 3

Crainic et al.(2015a) Marius Crainic, João Nuno Mestre and Ivan Struchiner. Deformations of lie groupoids. arXiv preprint arXiv:1510.02530. Cited in p. 3, 8, 11, 12, 14, 28, 29, 34, 39, 68

Crainic et al.(2015b) Marius Crainic, Maria Amelia Salazar and Ivan Struchiner. Multiplicative forms and spencer operators. Mathematische Zeitschrift, 279(3-4):939-979. Cited in p. 68

del Hoyo and Fernandes(2016) Matias del Hoyo and Rui Loja Fernandes. Riemannian metrics on differentiable stacks. arXiv preprint arXiv:1601.05616. Cited in p. 9

del Hoyo and Ortiz(2016) Matias del Hoyo and Cristian Ortiz. Morita equivalences of vector bundles. arXiv preprint arXiv:1612.09289. Cited in p. 39, 42

Grabowski and Rotkiewicz(2009) Janusz Grabowski and Mikolaj Rotkiewicz. Higher vector bundles and multi-graded symplectic manifolds. Journal of Geometry and Physics, 59(9):1285-1305. Cited in p. 59

Gracia-Saz and Mehta(2010a) Alfonso Gracia-Saz and Rajan Amit Mehta. Lie algebroid structures on double vector bundles and representation theory of lie algebroids. Advances in Mathematics, 223(4):1236-1275. Cited in p. 51, 59, 60

Gracia-Saz and Mehta(2010b) Alfonso Gracia-Saz and Rajan Amit Mehta. Vb-groupoids and representation theory of lie groupoids. arXiv preprint arXiv:1007.3658. Cited in p. 39, 41

Karasev(1989) MV Karasev. The maslov quantization conditions in higher cohomology and analogs of notions developed in lie theory for canonical fibre bundles of symplectic manifolds. i, ii. Selecta Math. Soviet, 8(3):213-234. Cited in p. 1

Kodaira(2006) Kunihiko Kodaira. Complex manifolds and deformation of complex structures. Springer. Cited in p. 2

Lee(1974) Dong Hoon Lee. On deformations of homomorphisms of locally compact groups. Transactions of the American Mathematical Society, pages 353-361. Cited in p. 3, 36

Li-Bland and Ševera(2011) David Li-Bland and Pavol Ševera. Quasi-hamiltonian groupoids and multiplicative manin pairs. International Mathematics Research Notices, 2011(10):2295-2350. Cited in p. 40

Mackenzie and $\mathrm{Xu}(\mathbf{1 9 9 7})$ Kirill Mackenzie and Ping Xu. Classical lifting processes and multiplicative vector fields. arXiv preprint $d g$-ga/9710030. Cited in p. 12

Mackenzie(2005a) Kirill CH Mackenzie. General theory of Lie groupoids and Lie algebroids, volume 213. Cambridge University Press. Cited in p. 39, 54, 55, 58, 61, 64 
Mackenzie(2011) Kirill CH Mackenzie. Ehresmann doubles and drinfel'd doubles for lie algebroids and lie bialgebroids. Journal für die reine und angewandte Mathematik (Crelles Journal), 2011(658):193-245. Cited in p. 59

Mackenzie(2005b) Kirill CH Mackenzie. Duality and triple structures. Em The breadth of symplectic and Poisson geometry, pages 455-481. Springer. Cited in p. 54

Mackenzie et al.(1994) Kirill CH Mackenzie, Ping Xu et al. Lie bialgebroids and poisson groupoids. Duke Mathematical Journal, 73(2):415-452. Cited in p. 70, 71

Mackenzie(1998) Kirill Charles Howard Mackenzie. Double lie algebroids and the double of a lie bialgebroid. arXiv preprint math/9808081. Cited in p. 51

Marcut(2013) Ioan Marcut. Normal forms in poisson geometry. arXiv preprint arXiv:1301.4571. Cited in p. 2

McDuff and Salamon(1998) Dusa McDuff and Dietmar Salamon. Introduction to symplectic topology. Cited in p. 2, 11, 31

Moerdijk and Mrcun(2003) Ieke Moerdijk and Janez Mrcun. Introduction to foliations and Lie groupoids, volume 91. Cambridge University Press. Cited in p. 8

Nijenhuis(1968) Albert Nijenhuis. Composition systems and deformations of subalgebras. Em Indagationes Mathematicae (Proceedings), volume 71, pages 119-136. Elsevier. Cited in p. 3

Nijenhuis and Richardson Jr(1966) Albert Nijenhuis and Roger W Richardson Jr. Cohomology and deformations in graded lie algebras. Bulletin of the American Mathematical Society, 72(1):1-29. Cited in p. 2

Nijenhuis and Richardson $\operatorname{Jr}(\mathbf{1 9 6 7})$ Albert Nijenhuis and Roger W Richardson Jr. Deformations of homomorphisms of lie groups and lie algebras. Bulletin of the American Mathematical Society, 73(1):175-179. Cited in p. 3, 31

Richardson et al.(1967) Roger W Richardson et al. A rigidity theorem for subalgebras of lie and associative algebras. Illinois Journal of Mathematics, 11(1):92-110. Cited in p. 3

Richardson Jr et al.(1969) RW Richardson Jr et al. Deformations of subalgebras of lie algebras. Journal of Differential Geometry, 3(3-4):289-308. Cited in p. 3

Weinstein(1996) Alan Weinstein. Groupoids: unifying internal and external symmetry. Notices of the AMS, 43(7):744-752. Cited in p. 1

Weinstein(1987) Alan Weinstein. Symplectic groupoids and poisson manifolds. Bulletin of the American mathematical Society, 16(1):101-104. Cited in p. 1

Zakrzewski(1990) Stanislaw Zakrzewski. Quantum and classical pseudogroups. part i. union pseudogroups and their quantization. Communications in Mathematical Physics, 134(2): 347-370. Cited in p. 1 